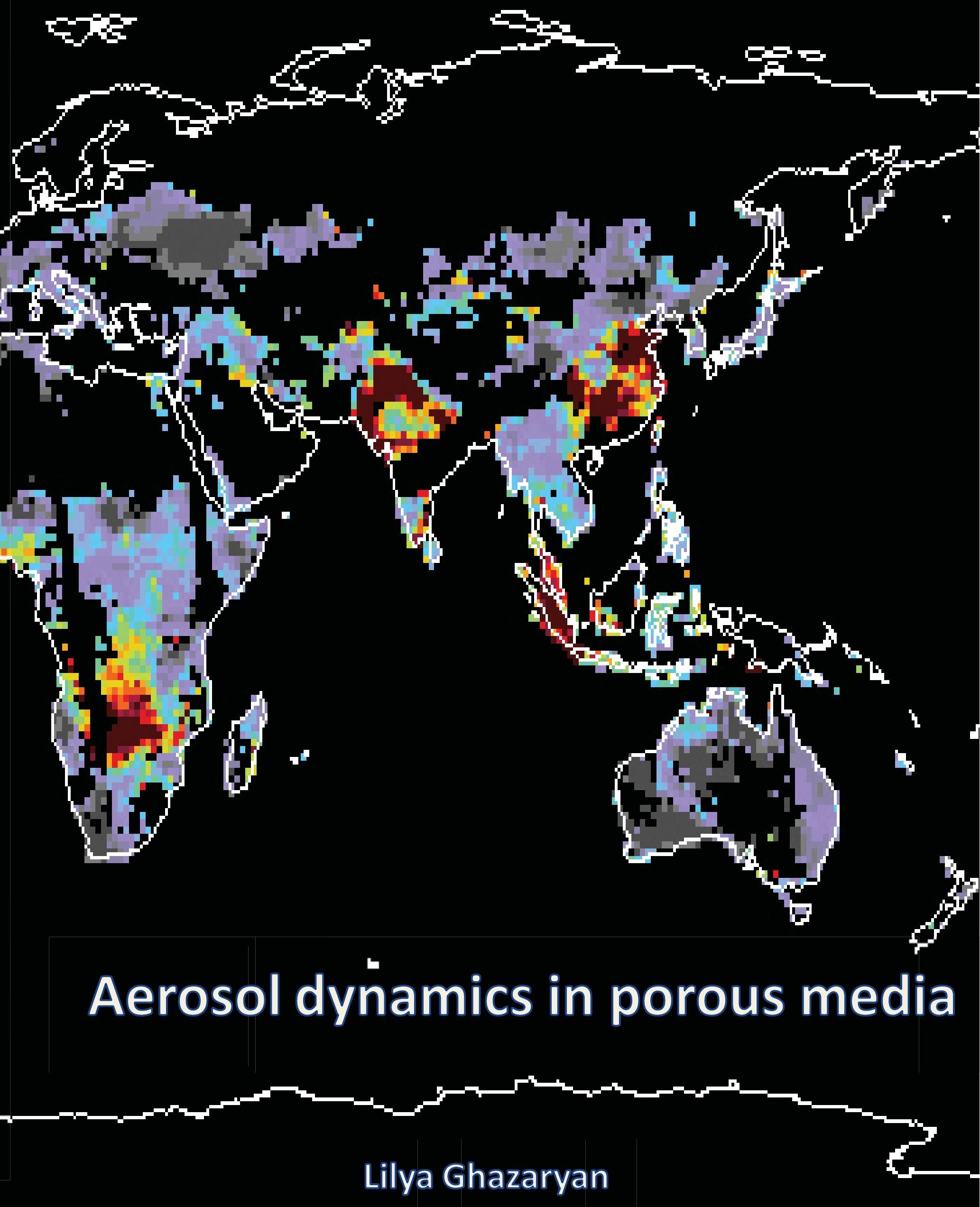


AEROSOL DYNAMICS IN POROUS MEDIA

Lilya Ghazaryan 
The research presented in this thesis was carried out at the chair of Multiscale Modeling and Simulation of the

Department of Applied Mathematics, University of Twente, P.O. Box 217, 7500 AE Enschede, The Netherlands.

\section{MESA+ \\ INSTITUTE FOR NANOTECHNOLOGY UNIVERSITY OF TWENTE.}

This research was supported by

Philip Morris Products S.A.,

Quai Jeanrenaud 5, 2000 Neuchatel, Switzerland.

Aerosol dynamics in porous media

Cover photo: generated by Terra and Aqua MODIS products available at: http://modis-atmos.gsfc.nasa.gov/IMAGES/index.html Copyright (c) 2014 by L. Ghazaryan

ISBN 978-90-365-3790-2

Printed by Wöhrmann Print Service, Zutphen, The Netherlands 


\title{
AEROSOL DYNAMICS IN POROUS MEDIA
}

\section{PROEFSCHRIFT}

\author{
ter verkrijging van \\ de graad van doctor aan de Universiteit Twente, \\ op gezag van de rector magnificus, \\ prof. dr. H. Brinksma, \\ volgens besluit van het College voor Promoties \\ in het openbaar te verdedigen \\ op donderdag 27 november 2014 om 14:45 uur
}

door

Lilya Ghazaryan

geboren op 3 augustus 1984

te Yerevan, Armenië 
Dit proefschrift is goedgekeurd door de promotor:

prof.dr.ir. B.J. Geurts 


\section{Summary}

In this thesis, a computational model was developed for the simulation of aerosol formation through nucleation, followed by condensation and evaporation and filtration by porous material. Understanding aerosol dynamics in porous media can help improving engineering models that are used in various industrial, medical and environmental applications. Within the Euler-Lagrange framework of modeling two-phase flow, the trajectories of individual aerosol droplets, as well as the heat and mass transfer with their surroundings, were evaluated in velocity, temperature and species concentration fields that were computed by applying the immersed boundary (IB) method to flow in complex domains.

Focusing mainly on rather dilute situations the so-called 'one-way' coupling approximation was adopted that allows to separate the problem of determining the flow field from the problem of tracking the motion of inertial droplets in that flow field. Following this approach, in Chapters 2 and 3 we concentrated on the problem of filtration of droplets by porous filters. First, we focused our attention on particle deposition on the solid filter surface due to inertial impaction. A numerical approach was described to simulate the motion of a large number of particles suspended in a gas flow that avoids numerical filtration of massless/passive particles. We considered two structured porous media in 3D, composed of in-line and staggered arrangements of square rods. It was established that the inner structure of a porous medium strongest influences the deposition of particles. In staggered geometries filtration appeared to depend strongly on particle inertia suggesting that the staggered geometry can be used to separate particles according to their Stokes number.

The 'no-slip consistent' particle tracking described in Chapter 2 is formulated entirely in terms of the phase-indicator function related to the inner structure of the filter. This enables adapting this method directly to more complex filter geometries which was done in Chapter 3, where we considered dynamics of droplets in a realistic porous filter. In this chapter, the dynamics of droplets was governed both by Stokes drag and Brownian motion. The effects of inertial motion and Brownian diffu- 
sion on the filtration characteristics were first illustrated for flow through a straight pipe. Subsequently, the filtration characteristics of a steady flow through a realistic porous material were determined, illustrating the potential of the approach in terms of predicting such macroscopic aspects based on pore-resolved flow. High filtration efficiency was observed in case of dominant Brownian motion or dominant inertial motion and a reduced filtration efficiency was found for droplets of intermediate size.

Filtration of already generated aerosol droplets can be regarded as an indirect way of controlling the aerosol that eventually emanates from a process. A more direct way implies control over the conditions at which the aerosol actually forms. This involves coupling of the fluid flow with the process of nucleation and subsequent evolution of the aerosol properties due to evaporation and condensation. We restricted ourselves to single-species aerosols and adapted the classical nucleation theory (CNT) which links locally supersaturated vapor state to the nucleation of so-called 'critical clusters'. The nucleation rate from CNT is adopted in the Euler-Lagrange framework as the probability per unit of time and volume to generate such critical clusters. Subsequent growth of a newly formed droplet can arise from further condensation of vapor molecules onto the droplet, thereby influencing the local vapor concentration and temperature fields. This computational model was applied to a laminar flow in a channel between two parallel plates. Nucleation was initiated by rapid cooling of air saturated with dibutylphthalate vapor at the inflow of the channel. Due to a sharp temperature drop at some location along the channel a supersaturated state is achieved, thereby inducing droplet nucleation. This approach illustrates a first application of the Euler-Lagrange framework to aerosol formation and presents aspects such as the evolving droplet size distribution and characteristics of the aerosol as it emanates from the end of the channel. It is a basis for studying the dependence of the aerosol formation process on important process parameters such as the temperature, the cooling rate and the flow velocity. 


\section{Samenvatting}

In dit proefschrift wordt een computermodel ontwikkeld voor de simulatie van het vormingsproces van aerosol door middel van nucleatie en de daaropvolgende processen van condensatie, verdamping en filtratie door poreus materiaal. Het begrijpen van het gedrag van aerosol in poreuze media draagt bij aan de verbetering van technische modellen voor diverse industriële, medische en ecologische toepassingen.

We hebben binnen het Euler-Lagrange raamwerk voor de modellering van tweefase stromingen de banen van individuele aerosoldruppels en ook de warmte- en massaoverdracht met hun omgeving geanalyseerd in snelheidsvelden, temperatuurvelden, en concentratie -velden van de diverse componenten in het mengsel. Deze velden zijn berekend door de zgn. 'Immersed boundary' methode toe te passen op stromingen in complexe domeinen. Door te focussen op situaties met lage aerosolconcentraties kan de zgn. 'one-way' koppelingsbenadering worden gebruikt. Hiermee kan het probleem van de bepaling van het stromingsveld worden gescheiden van het probleem van het volgen van de beweging van druppels in hetzelfde stromingsveld.

In hoofdstuk 2 en 3 hebben we ons geconcentreerd op het probleem van filtratie van druppels met behulp van poreuze filters. Allereerst, in hoofdstuk 2, hebben we ingezoomd op de afzetting van deeltjes op de oppervlakte van het filter door 'inertial impaction'. Vervolgens beschrijven we een numerieke aanpak om de beweging van een groot aantal deeltjes in een gasstroming te simuleren, waarbij 'numerieke filtratie' van passieve deeltjes wordt vermeden. We hebben twee poreuze media bestudeerd: de ene bestaande uit 'inline' en de andere uit 'staggered' geordende vierkante staven. Hieruit blijkt dat de inwendige structuur van het poreuze medium een grote invloed heeft op de afzetting van deeltjes. In de 'staggered' structuur hing de mate van filtratie sterk af van het impuls van de deeltjes. Dit suggereert dat deze structuur kan worden gebruikt om deeltjes te scheiden op basis van hun getal van Stokes.

De 'no-slip consistent particle tracking' methode beschreven in hoofdstuk 2 is volledig geformuleerd in termen van de 'phase-indicator' functie behorende bij de inwendige structuur van het filter. Hierdoor kan deze methode gemakkelijk worden 
aangepast aan complexere filters, zoals we hebben laten zien in hoofdstuk 3. Daar hebben we voor realistische poreuze filters het gedrag van druppels bestudeerd. Dit gedrag wordt voornamelijk bepaald door 'Stokes drag' en Brownse beweging. We hebben in eerste instantie het effect van impuls en Brownse diffusie op de filtratieeigenschappen laten zien voor stroming door een rechte pijp. Vervolgens hebben we de filtratie-eigenschappen ook bepaald voor een stabiele stroming door een realistisch poreus materiaal. Dit laatste toont het potentieel van onze aanpak met betrekking tot het voorspellen van macroscopische aspecten gebaseerd op een stroming berekend op de schaal van de poriën. Bijvoorbeeld voor de gevallen van dominante Brownse beweging en dominante impuls vinden we een hoge filtratie-efficiëntie, terwijl voor het geval van druppels van middelmatige grootte de filtratie-efficiëntie gereduceerd bleek.

Filtratie van reeds gegenereerde aerosoldruppels kan worden beschouwd als een indirecte methode om het aerosol dat uiteindelijk het proces verlaat te kunnen beheersen. Een directere methode impliceert dat de omstandigheden waaronder het aerosol ontstaat beter moeten worden beheerst. Dit vereist eerst het koppelen van de stroming aan het zowel nucleatieproces als aan het ontwikkelen van de aerosoleigenschappen door middel van verdamping en condensatie. In hoofdstuk 4 hebben we deze aanpak beschreven waarbij we onszelf beperkt hebben tot aerosol dat uit 1 enkele component bestaat. Voor deze situatie hebben we de klassieke nucleatie theorie (CNT) toegepast. Deze theorie relateert de lokale oververzadigde damptoestand aan de nucleatie van zgn. 'kritische clusters'. Binnen het Euler-Lagrange raamwerk hebben we de kans om per eenheid van tijd en volume zulke kritische clusters te genereren gelijk genomen aan de nucleatiesnelheid van de CNT. Het raamwerk biedt ook de mogelijkheid tot integratie van verdere groei van nieuwgevormde druppels met als gevolg veranderende lokale dampconcentraties en temperatuurvelden. Het onderliggende computermodel hebben we toegepast op een laminaire stroming in een kanaal tussen twee parallelle platen. Hierbij werd het nucleatieproces ge“ initieerd door het snel afkoelen van lucht verzadigd met dibutylftalaat bij de instroom van het kanaal. Door de snelle daling van de temperatuur wordt in het kanaal een oververzadigde toes- 
tand bereikt wat leidt tot de nucleatie van druppels. Dit demonstreert een eerste toepassing van het Euler-Lagrangian raamwerk op de vorming van aerosol en geeft de transiënte druppelgrootteverdeling en eigenschappen van het aerosol wanneer deze het kanaal verlaat. Daarmee vormt dit werk een basis om de afhankelijkheid van het vormingsproces van aerosol van belangrijke procesparameters zoals de temperatuur, de afkoelsnelheid en de stromingssnelheid verder te bestuderen. 


\section{Contents}

1 Introduction 13

2 No-slip consistent immersed boundary particle tracking to simulate $\begin{array}{ll}\text { impaction filtration in porous media } & 19\end{array}$

2.1 Introduction . . . . . . . . . . . . . . . . . . . 20

2.2 Motion of aerosol droplets in a gas flow . . . . . . . . . . . . . . 23

2.2.1 Governing equations for the gas phase . . . . . . . . . . . 23

2.2.2 Governing equations for the particle phase . . . . . . . . 26

2.3 Numerical simulation of gas-droplet two-phase flow through a porous

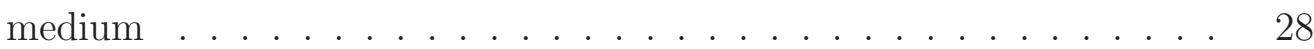

2.3.1 Immersed Boundary method . . . . . . . . . . . . . . . . . 28

2.3.2 Particle tracking . . . . . . . . . . . . . . . . . 32

2.3.3 Analysis of particle motion near a solid-fluid interface in 1D . 33

2.3.4 3D solid-fluid interface velocity interpolation . . . . . . . . . 37

2.4 Impaction filtration of aerosol droplets in porous media . . . . . . . . 39

2.4.1 The motion of particles in model porous media . . . . . . . . . 40

2.4.2 Impaction filtration of aerosol droplets in porous media . . . . 45

2.5 Conclusions . . . . . . . . . . . . . . . . . 53

3 Diffusive and inertial impaction filtration of aerosol droplets by porous media $\quad 55$

3.1 Introduction . . . . . . . . . . . . . . . . . . . 55 
3.2 Modeling the motion of small aerosol droplets due to inertial and random forces . . . . . . . . . . . . . . . . . . . . 58

3.2.1 Eulerian description of the gas-phase . . . . . . . . . . 58

3.2.2 Lagrangian description of the droplet-phase . . . . . . . . . 59

3.2.3 Numerical solution of governing equations . . . . . . . . . . 62

3.2.4 Validation of Brownian motion in 1D . . . . . . . . . . . 63

3.3 Filtration in a circular tube . . . . . . . . . . . . . 65

3.4 Filtration in a porous filter . . . . . . . . . . . . . . 70

3.4.1 Construction of the computational domain . . . . . . 70

3.4.2 Numerical prediction of the filtration efficiency . . . . . . . . 72

3.5 Conclusions . . . . . . . . . . . . . . . . . . . 80

4 Numerical simulation of aerosol formation and growth in laminar $\begin{array}{ll}\text { flow } & 83\end{array}$

4.1 Introduction . . . . . . . . . . . . . . . . . . . 83

4.2 Mathematical modeling . . . . . . . . . . . . . . . . . 85

4.2.1 Gas-vapor phase . . . . . . . . . . . . 86

$4.2 .2 \quad$ Droplet phase . . . . . . . . . . . . . . . . . 87

4.2.3 Coupling the discrete and the continuous phases . . . . . . . . 92

4.2.4 Numerical treatment . . . . . . . . . . . . . . . . . 95

4.3 Droplet formation and growth in a channel flow . . . . . . . . . . 96

4.3.1 Numerical experiment . . . . . . . . . . . . . . 97

4.3.2 Droplet generation and growth . . . . . . . . . . . . . 101

4.4 Conclusions . . . . . . . . . . . . . . . . . . . 106

5 Conclusions and Recommendations 107 


\section{Chapter 1}

\section{Introduction}

The concept of an aerosol refers to large numbers of tiny particles or droplets that are suspended in the air around us [23]. Their properties may influence our lives in a number of desired and sometimes undesired ways. One may think of soot particles emitted by Diesel engines [32], which may reside in the atmosphere for extended periods of time and be inhaled, constituting a health risk primarily in urban situations under conditions of serious smog $[45,24]$. Less risky at first sight may be aerosols consisting of fine dust. However, depending on the prevalent size of these particles, studies have shown that over time contamination even deep into our brains may occur, resulting from extended periods of exposure [52]. Another well-known aerosol present in our daily lives is formed by small water droplets, contributing to a hazy atmosphere of silvery 'Dutch light' at times, and known to be an important green-house gas by itself.

Apart from the presence of aerosols in the environment, there is a number of technological/medical applications of aerosols worth mentioning. A prime example is in the treatment of asthmatic patients in which an aerosol should be generated from the medication to penetrate the lungs [26, 27]. This requires the formulation of an aerosol with quite precise size distribution in order to guarantee that the effective substances contained in the droplets actually do reach deep into the lungs rather than get deposited early on in the airway tract, as would occur if the droplets would be too large $[33,59]$. As a final example of this certainly not comprehensive overview, 
the problem of spray drying can be mentioned in which a finely dispersed spray of, e.g., a food substance is formed and exposed to heat in order to rapidly evaporate the more volatile components. This process also requires considerable control over the formation of the aerosol such that the final product satisfies rather narrow quality conditions in terms of size and composition of the deposited 'dried' particles [1].

Within the field of aerosol research, this thesis is devoted to the mathematical modelling and simulation of the generation, evolution and filtration of aerosol droplets. To formulate accurate models for such aerosols a number of processes need to be dynamically coupled. On the one hand, the generation and evolution of the aerosol mainly relates to the nucleation of nano-meter sized critical clusters from a supersaturated vapor, followed by further condensation and evaporation of vapor onto and from the aerosol droplets, respectively. On the other hand, the filtration of droplets deals with the capturing of droplets in a flow by collision with solid objects contained in the domain. Both through a careful maintenance of the desired process conditions associated with the generation and transport of the aerosol, as well as through properly designed filtration, one may achieve a good level of control over the properties of the resulting aerosol. In this way one may prepare aerosols with a desired size distribution and chemical composition for subsequent use in various applications as sketched above.

To arrive at an accurate yet efficient mathematical description of an aerosol, one faces the dilemma of addressing the aerosol at the level of discrete particles and droplets embedded in a continuous gaseous carrier phase, or to coarsen the description and treat the large number of particles in the aerosol as a continuum as well, capturing their distribution in terms of a space and time dependent density field. The latter would be referred to as an Euler-Euler formulation while the former is known as an Euler-Lagrange framework, with the dispersed discrete particulate phase associated with the Lagrangian part of the description. These two formulations obviously have different advantages and drawbacks. Therefore, depending on the precise application a choice for either needs to be made. Generally, the Euler-Euler framework is more suitable for large-scale applications as it is computationally more accessible, while the 
Euler-Lagrange framework requires, as a rule, more computational resources, up to the point of becoming not feasible for realistic conditions with correspondingly large numbers of particles. However, the Euler-Lagrange framework is often seen as the more fundamental approach of the two, allowing to introduce specific mechanisms into the model at a more basic and often better controlled level of accuracy. As an example, while the presence of phase transition in the Euler-Euler framework requires the inclusion of phenomenological source terms to couple vapor and liquid phases, the Euler-Lagrange framework would allow such heat and mass transfer on the basis of a more microscopic and often more fully motivated set of assumptions.

An Euler-Euler approach requires coupling of fluid flow to the transport of energy and of aerosol-forming vapor and its liquid phase. The generation and evolution of aerosol droplets, accounting for the phase transitions, requires the inclusion of source terms for energy transfer, nucleation and evaporation/condensation. While the transport part of such a model follows from well-established conservation principles, the source terms are formulated on the basis of phenomenological classical nucleation theory (CNT) [57, 3]. We will consider only single-species aerosol formation, e.g., from water vapor, for which CNT provides a complete physical description with a good level of accuracy. The Euler-Euler approach can be complemented with the EulerLagrange formulation in which fluid, vapor and energy transport are described by a system of partial differential equations, while the dynamics of the aerosol droplets, treated as point particles, is captured by a system of ordinary differential equations governing the motion of individual droplets as well as aspects such as the droplet temperature and its size. The link with CNT is provided in terms of the probability of actually initiating a droplet of specified initial size, taken directly from the CNT source terms. In particular, the nucleation rate may be interpreted as this probability per unit volume and unit time, which can be evaluated along with a simulation of the developing flow, thereby completing the Euler-Lagrange formulation.

Apart from control over the properties of an aerosol by controlling the conditions at which the aerosol was formed, i.e., control at the source, one may also exert some level of control by filtration in which an already formed aerosol is passed through a 
rather complex domain which is designed such that droplets or particles of a certain size range are more likely to pass the filter than particles which are considerably smaller or larger $[14,47,12,51,18,43,6,4,9,19]$. In order to investigate filtration characteristics of flow through such complex domains, a prime challenge is to accurately predict the flow down to details of the size of individual pores in a filter. For this purpose, in computational fluid dynamics one distinguishes between bodyfitted grids and immersed boundary methods. In the body-fitted grid approach much effort is put into generation of a smooth grid suitable for accurate flow simulations in the fluid-filled pores. Such a grid aligns precisely with the fluid-solid interface. Obviously, the generation of such a grid becomes more and more demanding with increasing complexity of the inner structure of the porous material. Alternatively, we will adopt a so-called immersed boundary (IB) method [36, 2, 49] in which the fluid and solid regions are represented on a simple Cartesian grid. In fact, a grid cell is assigned to be of type 'solid' in case its contents is for more than $50 \%$ solid and of type 'fluid' otherwise. In this way a 'staircase' approximation of the fluid-solid interface arises and the flow that takes place in the fluid-filled regions can be simulated. This approach was employed to the level that actual tomographic reconstructions of a given porous material could be used as basis for the domain representation and a full capturing of flow in such domains was possible [30, 35].

The problem of filtration of aerosol droplets may be expressed conveniently in the Euler-Lagrange framework. In fact, in the dilute regime, i.e., in case of oneway coupling between the flow of air and the motion of the embedded droplets, the immersed boundary method can be adopted to independently compute the detailed flow field. For steady flows one may subsequently compute the motion of droplets of different inertia in this flow. As inertia implies that droplet trajectories do not coincide with streamlines of the flow, the droplets may actually collide with objects present in the domain. Such collision is required in order to have the possibility of filtration of droplets by deposition on these objects. Taking the simple rule that any encounter of a droplet with the surface of an object implies deposition then gives a basic algorithm to compute filtration efficiency of a given configuration of objects. 
This can be applied to rather simple configurations and serve as general validation of a computation, but also to flow domains of realistic complexity such as derived from fibrous materials. In this way one may predict aspects as the overall filtration efficiency but also local information such as the locations in the domain where most filtration occurs. While the overall filtration efficiency may be compared with experimental findings, predictions of the precise motion and deposition of droplets on the walls of a complex flow domain are examples of complementary information that is currently only accessible through simulation.

The organization of this thesis is as follows:

- Chapter 2 is devoted to the formulation and application of a new treatment of near-wall motion of inertial droplets transported by Stokes drag in a flow predicted using the staircase approximation IB approach as sketched above. The new treatment is designed to be consistent with the fact that in the limit of very small droplets without inertia, collision with solid objects in a flow will not occur. This is essential in order to prevent a component of unphysical filtration due to numerical discretization errors in case of droplets with low Stokes numbers and is basic to all Euler-Lagrange simulations presented in this thesis. In this chapter, we investigate the filtration characteristics of a staggered array of beams in case droplets are transported in laminar flow at low Reynolds numbers and quantify the dependence of filtration on the droplet sizes.

- Chapter 3 considers filtration of droplets whose dynamics is governed by both Stokes drag as well as Brownian motion. In case of sufficiently large inertia, i.e., sufficiently large Stokes numbers, the consequences of Brownian forces acting on the droplets will be negligible. However, as the droplet inertia reduces with droplet size, Brownian forces will become more important at typical process temperatures and even induce strong diffusive transport in the limit of very small droplets. The competition between inertial motion and Brownian diffusion has marked effects on the filtration characteristics. This is first illustrated for flow through a straight pipe, serving also as validation of the model. Sub- 
sequently, the filtration characteristics of steady flow through a realistic porous material are determined, illustrating the capabilities of the approach in terms of predicting such macroscopic aspects based on pore-resolved flow. In fact, we show a characteristic 'V-shaped' dependence of the filtration efficiency on the droplet size with high values in case of dominant Brownian motion or dominant inertial motion and a strongly reduced filtration efficiency for droplets of intermediate size.

- Chapter 4 deals with a first Euler-Lagrange model of the full set of processes of aerosol nucleation, growth by condensation and evaporation, and motion, governed by Stokes drag as well as Brownian forces. This model is applied to Poiseuille flow. The flow contains vapor at the inflow, which is rapidly cooled at some location downstream, thereby inducing droplet nucleation. While work presented in literature mainly deals with an Euler-Euler formulation [17, 42] in Chapter 4 we show results based on the more fundamental Euler-Lagrange formulation in which the trajectories of nucleated droplets are followed precisely in time. In this way we may directly predict the size distribution and other statistical properties of the aerosol as it exits the tube, and hence predict what level of control one may exert on the aerosol by adapting flow conditions and cooling parameters.

- Chapter 5 presents the main conclusions of the research and a set of recommendations for future investigations. 


\title{
Chapter 2
}

\section{No-slip consistent immersed}

\section{boundary particle tracking to}

\section{simulate impaction filtration in}

\author{
porous media ${ }^{1}$
}

\begin{abstract}
In this chapter we present a new method for simulating the motion of a disperse particle phase in a carrier gas through porous media. We assume a sufficiently dilute particle-laden flow and compute, independently of the disperse phase, the steady laminar fluid velocity using the Immersed Boundary (IB) method. Given the velocity of the carrier gas, the equations of motion for the particles experiencing the Stokes drag force are solved to determine their trajectories. The 'no-slip consistent' particle tracking algorithm avoids possible numerical filtration of very small particles due to the non-zero velocity field at the solid-fluid interface introduced by the IB method. This physically consistent tracking allows a reliable estimation of the filtration efficiency of porous filters due to inertial impaction. We illustrate and test our new approach for model porous media consisting of a structured array of aligned rectangular fibers, arranged in-line and staggered. In the staggered geometry the effect of the residual velocity at the solid-fluid interface is significant for particles with low inertia. Without adopting the developed 'no-slip consistent' numerical method, an artificial numerical filtration is observed, which becomes dominant for small enough particles. For both
\end{abstract}

\footnotetext{
${ }^{1}$ This chapter was published as: Ghazaryan, L., Lopez Penha, D.J., Stolz, S., Kuczaj, A.K., Geurts, B.J.: 2013. No-slip consistent immersed boundary particle tracking to simulate impaction filtration in porous media, International Journal for Numerical Methods in Fluids, 73 (7), 615-636
} 
the in-line and the staggered geometries the filtration rate depends quite strongly and non-monotonically on the particle inertia. This is expressed most clearly in the staggered arrangement in which a very strong increase in the filtration efficiency is observed at a well-defined critical droplet size, corresponding to a qualitative change in the dominant particle paths in the porous medium.

\section{$2.1 \quad$ Introduction}

In many practical applications porous media are used for separation of particulate matter from gasses, for example for air purification from dust particles, allergens or viruses. Prediction of particle deposition on the solid filtering material is an essential component in developing suitable filtration techniques. Several theoretical $[14,47,12]$, numerical $[51,18,43,6]$ and experimental $[4,9,19]$ approaches have been developed to quantify and qualify the filtration efficiency of porous filters. Theoretical and numerical approaches are usually limited to certain geometries while experimental measurements are often difficult to perform. In this contribution, we present a method that allows to simulate, from first principles, filtration properties of filters with known complex inner structures down to the pore scale, e.g., from microcomputed tomography $(\mu \mathrm{CT})$ [50]. The method can be used not only to estimate the filtration efficiency of given filters, but also to study the influence of a number of relevant physical and geometrical parameters on the particle deposition.

The deposition of particles on the surface of a filter can be caused by different filtration mechanisms, including inertial impaction and diffusion [23]. In both cases, particles are captured because their trajectories deviate from fluid streamlines close to the surface of the solid. In the case of impaction this is due to the particle inertia while in the case of diffusion the underlying Brownian motion is the cause. Depending on the particle inertia one of these mechanisms is dominant. Particle inertia, or the particle's responsiveness to changes in the flow, is characterized by the Stokes number $(S t)$ : the ratio of the particle response time to the flow time scale. In the absence of Brownian motion, low inertia particles essentially follow the fluid flow and therefore are transported as fluid elements. Relatively larger particles can, e.g., be trapped by 
vortices in the flow. Trajectories of particles with large inertia will not be affected much by vortex structures, due to the large inertial resistance to changes in the flow.

An important step to compute the inertial filtration component properly, concerns the treatment of particles with low inertia. While particles with considerable inertia can be readily tracked on their way to collision with the solid surface, tracking light particles may lead to an exaggerated filtration due to the residual velocity at the solid-fluid interface, introduced by our numerical method. The key is to develop a particle tracking method that ensures that also at finite numerical resolution, massless particles will not collide at all with the solid surface, but follow the intricate motion around the surface.

Our aim is to develop a proper numerical method for particle tracking that is physically consistent for the entire range from heavy to very light particles. For this purpose the point-particle approximation is very suitable, allowing to evaluate the trajectories of individual particles at low computational cost $[44,58]$. For resolving the gas flow we use a symmetry-preserving finite-volume discretization on a staggered grid [56]. In order to incorporate the solid material we employ an Immersed Boundary (IB) method, which allows one to consider complex porous media [36]. Alternative to body-fitted meshes, in IB methods simple Cartesian meshes are used and the solution algorithm is locally modified with a forcing term to represent the physical boundaries. IB methods are also advantageous when dealing with problems with moving boundaries [21, 60], multi-phase or multi-material problems [16]. A number of studies address issues specific to the treatment of flow near the boundary representation, discussing various aspects of discretization on cut cells [55, 11, 15, 53, 39]. In this paper the issue of mass conservation at the immersed boundary is treated in the context of particle tracking. The IB resolution of the gas flow on the staggered grid results in small, but nonzero 'residual' velocities at the solid-fluid interfaces. This residual velocity plays an important role in the Lagrangian tracking of particles, and it may imply deposition of massless particles on the solid surface. We formulate a tracking method constructed explicitly such that massless particles do not get captured. This ensures that deposition estimated for very small particles is not increased due to fil- 
tration arising from numerical errors. This will yield a more accurate estimation of filtration due to inertial impaction.

The proposed approach is studied for in-line and staggered structured porous media in 3D $[29,38,31]$. The effect of the residual velocity at the solid-fluid interface is essential for low inertia particles as this dominates the estimated filtration and leads to large relative errors in the estimated filtration efficiency. High inertia particles are less sensitive to these residual velocities.

Based on our approach for simulating the motion of particles in a gas flow through porous material, filtration characteristics can be predicted for any complex porous medium. The method allows one to understand filtration efficiency in terms of the pore-scale motion that arises. Particle deposition both in the in-line and the staggered geometries showed to depend on the particle size. In the staggered geometry different filtration regimes can be identified, depending on the particle inertia. A first regime is found for sufficiently small particles, which is characterized by a low removal rate, implying particles to be transported through the porous medium without being captured much. On the contrary, higher inertia particles get filtrated very efficiently. This property of the staggered geometry can be adopted for filter design, for instance, to separate particles with different sizes. The precise dependence of the filtration efficiency on the particle size and inner structure of the porous medium is quite complex. The new simulation method allows one to determine the filtration characteristics with high accuracy, using an Euler-Lagrange approach in which individual particles are tracked as they move through the flow domain.

In Section 2.2, we describe the mathematical models for the particle-laden flow of the carrier gas through a porous medium. Next, in Section 2.3, the numerical treatment applied in our model is discussed. Here, we mainly address the computational technique developed to avoid possible numerical filtration of very small particles. Section 2.4 is devoted to the application of the method to investigate filtration properties of structured porous media for different particle sizes. We summarize our findings in Section 2.5. 


\subsection{Motion of aerosol droplets in a gas flow}

In this section we describe the Euler-Lagrange modeling of gas-particle two-phase flow, using the point particle approximation for the particle motion. First we turn to the gas flow and afterwards describe the particle phase.

\subsubsection{Governing equations for the gas phase}

The gas flow is treated on the basis of mass and momentum conservation. In our temporal simulations, the governing equations are the incompressible Navier-Stokes equations. We assume that the periodic physical domain $\Omega$ contains 'obstacles', which form the solid part $\Omega_{s}$ of our computational domain. The non-dimensional NavierStokes equations read, in vector form [44]:

$$
\left\{\begin{aligned}
\frac{\partial \mathbf{u}}{\partial t}+\mathbf{u} \cdot \nabla \mathbf{u} & =-\nabla p+\frac{1}{R e} \nabla^{2} \mathbf{u} \\
\nabla \cdot \mathbf{u} & =0
\end{aligned}\right.
$$

in the flow domain $\Omega_{f}$. This set of equations describes the flow of fluid through the pores that are left by the solid material. The variables to be solved for are the velocity components $\mathbf{u}=(u, v, w)$ and the 'reduced pressure' $p$ arising from the nondimensionalization of the term $P / \rho_{f}$ that appears in the Navier-Stokes equations, where $P$ is the actual pressure and $\rho_{f}$ the mass density of the fluid. In order to maintain a constant volumetric flow rate through the domain the nondimensional reduced pressure $p$ is expressed as: $p=a(t) x+\widetilde{p}$ where $a(t)$ is the mean pressure gradient in the stream-wise $x$ direction, forcing a prescribed constant volumetric flow through the domain and $\widetilde{p}$ represents fluctuations relative to the linear background pressure field. The fluctuations $\widetilde{p}$ are assumed to be periodic in space, a step commonly made when dealing with fluid flow in periodic domains [37]. The Reynolds number $R e$ characterizes the ratio of convective and viscous fluxes and is defined as:

$$
R e=\frac{L U}{\nu}
$$


where $\nu$ is the kinematic viscosity and $L$ and $U$ are characteristic length and velocity scales, respectively. These scales also introduce a natural time-scale $L / U$ and will be used when considering the motion of suspended particles. In addition to the equations, no-slip boundary conditions have to be satisfied at all solid-fluid interfaces, i.e., at any solid boundary the fluid will have zero relative velocity.

Numerical methods for solving the Navier-Stokes equations around obstacles ([44], [13]) are classified according to whether the computational grid is (a) body-conforming, with grid-lines closely following the solid-fluid interface, or (b) non body-conforming, with the obstacles 'immersed'. In the past decades, a number of IB methods has been used for simulation of flow through complex porous materials. The goal of these methods is to avoid the expensive construction of body-conforming grids. Here, we use the IB method [36], employing Cartesian grids, on which efficient and fast numerical methods can be used. The flexibility of IB methods allows one to use any geometry given by, for example, micro-computed tomography [30], without applying difficult meshing techniques needed for body-conforming grids. A challenge to any numerical method is the representation of flow near the actual solid boundary. If the complexity of the domain allows a body-fitted grid then the imposition of the boundary conditions can be done accurately and at the correct location. Alternatively, IB methods can only address accurate treatment of the boundary conditions by adopting adequate spatial resolution in combination with first or second order accuracy near the boundary [49]. A representative IB method is the so-called volume penalization method [2]. The idea is the following: instead of solving the problem in the fluid domain $\Omega_{f}$, an extended problem on the whole domain is solved by penalizing the flow entering the obstacles. This is done by adding a solution-dependent source term to the momentum equations:

$$
\left\{\begin{aligned}
\frac{\partial \mathbf{u}}{\partial t}+\mathbf{u} \cdot \nabla \mathbf{u} & =-\nabla p+\frac{1}{R e} \nabla^{2} \mathbf{u}-\frac{1}{\epsilon} \Gamma_{s} \mathbf{u} \\
\nabla \cdot \mathbf{u} & =0
\end{aligned}\right.
$$

which applies to all $\mathbf{x}$ in the domain $\Omega$. The penalization parameter $\epsilon \ll 1$ and $\Gamma_{s}$ is the characteristic function of $\Omega_{s}$ (the solid part of the domain), which we refer to as 
phase-indicator function. It is defined as:

$$
\Gamma_{s}(x)=\left\{\begin{array}{lll}
1 & \text { if } & x \in \Omega_{s} \\
0 & \text { if } & x \in \Omega_{f}
\end{array}\right.
$$

The IB approach allows to compute the flow field through porous media with very complex inner geometries, provided the resolution is adequate. For validation and illustration purposes we consider two structured porous media. Cross sections of these two model porous media are shown in Figure 2-1. Both porous media are constructed using a combination of square rods of size $L \times L$ in $x$ and $y$ directions and infinitely extended in the $z$ direction. The porous medium given in Figure 2-1(a) arises by arranging the squares in-line [38], while in Figure 2-1(b) a staggered arrangement is chosen [29]. In this paper, we will refer to these two structured porous media as in-line and staggered, respectively. The corresponding representative elementary volume (REV), which serves as building block for these porous media, is indicated by the dashed lines in Figure 2-1. By changing the distance between the squares the volume occupied by the solid can be changed giving control over the porosity [41]. We assume that the distance between the squares in both $x$ and $y$ directions is $L$, implying porosity of $3 / 4$. Both the in-line and the staggered porous media were used in Lopez Penha et al. [31] for validation of the IB method. Including both the in-line and the staggered arrangement allows to study the influence of the inner flow structure on the particle deposition.

Typically, the gas flow velocity characterizing the bulk flow in common filters, termed the face velocity, is on the order of $0.1 \mathrm{~m} / \mathrm{s}$ [23]. For our investigation of the particle deposition we will consider $R e=100$, which roughly corresponds to typical industrial filters with a characteristic length scale in the order of $15 \mathrm{~mm}$ using the kinematic viscosity of air at ambient conditions, i.e., $\nu=15.11 \times 10^{-6} \mathrm{~m}^{2} / \mathrm{s}$. This yields a typical time scale $L / U$ of order 0.1 seconds. The flow field corresponding to this value of the Reynolds number is laminar and develops several vortical flow structures in the wakes of the rods [31]. 


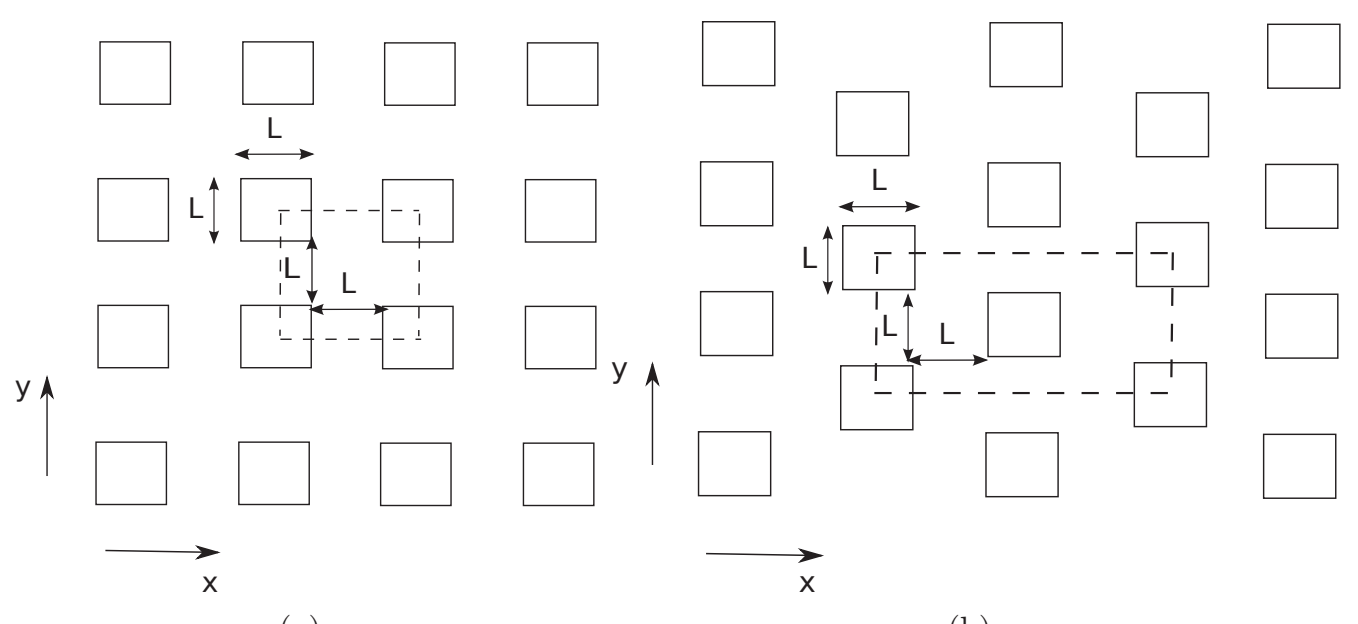

(a)

(b)

Figure 2-1: 2D cross-sectional plots of in-line (a) and staggered (b) arrangement of square rods.

\subsubsection{Governing equations for the particle phase}

The dynamics of a large number of independently moving particles, suspended in the flow, is obtained by evaluating the solutions of the equation of motions that involves the forces acting on the particles. We consider aerosol particles consisting of water suspended in air, which implies that the ratio of the particle density $\rho_{p}$ and the gas density $\rho_{f}$ is of the order $10^{3}$. This allows to consider dynamics due to Stokes drag alone, thereby greatly simplifying the general equation of motion given by MaxeyRiley [34]. Taking into account the kinematic relationship for the particle position, the total description of the motion of an individual particle is given by:

$$
\left\{\begin{array}{l}
\frac{\mathrm{d} \mathbf{x}}{\mathrm{d} t}=\mathbf{v} \\
\frac{\mathrm{d} \mathbf{v}}{\mathrm{d} t}=\frac{1}{S t}(\mathbf{u}(\mathbf{x}(t), t)-\mathbf{v}(t))
\end{array}\right.
$$

where $\mathbf{v}$ is the particle velocity and $\mathbf{u}(\mathbf{x}(t), t)$ is the fluid velocity at the actual particle position $\mathbf{x}(t)$. In this equation of motion for a particle, the key parameter is the Stokes number St. This number is obtained by scaling the particle response time $\tau$ by the time-scale $L / U$ as introduced before for the non-dimensionalization of the 
Navier-Stokes equations. The response time expresses the timescale with which the particle's relative velocity goes to zero in the absence of an external flow [23], [7]. The particle response time depends on its diameter $D$, the density of the particle $\rho_{p}$ and the molecular viscosity of the carrier gas $\mu$ :

$$
\tau=\frac{\rho_{p} D^{2}}{18 \mu}=\frac{D^{2}}{18 \nu} \frac{\rho_{p}}{\rho_{f}}
$$

where in the latter expression we emphasize the dependence on the ratio of the particle and the fluid mass density, and introduce the kinematic viscosity of the gas $\nu$. The dimensionless Stokes number is defined as:

$$
S t=\tau \frac{U}{L}=\frac{1}{18} \frac{\rho_{p}}{\rho_{f}} \frac{D}{L} \frac{U D}{\nu}=\frac{1}{18} \frac{\rho_{p}}{\rho_{f}} \frac{D}{L} R e_{p}
$$

where $R e_{p}$ is the Reynolds number based on the diameter of the particle. When considering particles consisting of water with a size-range $0.1 \mu m \leq D \leq 40 \mu m$ in air, we find the response time values $4 \cdot 10^{-8} s \leq \tau \leq 6 \cdot 10^{-3} s$. In the context of filtration considered before, a typical time-scale is 0.1 seconds, yielding values for the Stokes number as follows: $4 \cdot 10^{-7} \leq S t \leq 6 \cdot 10^{-2}$. For the range of particle diameters $D \geq 0.1 \mu m$ impaction becomes important. Particles with size below $0.1 \mu m$ are not likely to be captured due to impaction and their deposition due to this mechanism is negligible or not present at all [23]. Preserving this property of small particles on the numerical level is essential.

Following the trajectories of the particles, we can investigate the dependence of their motion on a number of parameters, such as the droplet size, the flow, etc. The Stokes number acts as a measure of inertia containing a $D^{2}$ dependence on the size of the particle. A larger value of the Stokes number implies a higher resistance to a sudden change in the flow. Conversely, if the Stokes number of a particle is small enough, it easily adopts to any changes in the flow. In the limit $S t \rightarrow 0$, which describes a particle without inertia (massless particle), the particle perfectly follows the streamlines of the fluid flow, and hence would not collide with the solid material making up the filter. For an accurate prediction of the particle dynamics in the range 
of low Stokes numbers, this property of a massless particle has to be preserved also for the numerically computed trajectories. While particles with a nonzero St can follow trajectories that could lead to a collision with one of the solid-fluid interfaces in the domain, massless $S t=0$ particles should never encounter such collisions. This is a crucial aspect to maintain numerically, since it is of direct relevance to particle filtration efficiency at low St. Next we present a numerical particle tracking method for IB simulations that is fully consistent with this requirement.

\subsection{Numerical simulation of gas-droplet two-phase flow through a porous medium}

The system of equations formulated in the previous section is solved numerically. In the following subsections we will describe the discretization method for solving the Navier-Stokes equations. The particle tracking in the velocity field will be presented in which we detail our approach for computing a particle's velocity close to the solidfluid interface.

\subsubsection{Immersed Boundary method}

In the previous section we described the set of governing equations for the gas-phase. We use an IB method to compute the gas flow around solid obstacles embedded in the domain. The IB technique employs Cartesian meshes on which the governing equations are solved. Once the Cartesian mesh $\left\{x_{i}, y_{j}, z_{k}\right\}$, with $i \in[1, \ldots, n x], j \in$ $[1, \ldots, n y]$, and $k \in[1, \ldots, n z]$ is defined, the next step is to discretize the governing equations (3.1) on this mesh. Below we will detail the discretization method and describe the representation of the solid-fluid interface.

For simulation of incompressible flow a finite-volume discretization of the equations (3.1) is used [44]. In collocated finite-volume methods, the control volumes, over which the Navier-Stokes equations are integrated, are the same for the different velocity components. Here, we consider uniform Cartesian grids with different control 
volumes for the different components of velocity, which results in a staggered storage of variables. An illustration is given for the 2D case in Figure 2-2. The control volume $\mathbf{V}_{i+1 / 2, j}$ for the velocity component in the $x$-direction, $u$, is staggered from the 'basic' control volume $\mathbf{V}_{i, j}=\left[x_{i-1}, x_{i}\right] \times\left[y_{j-1}, y_{j}\right]$ to the right direction by half a grid cell. If we denote by $x_{i+1 / 2}=\left(x_{i}+x_{i+1}\right) / 2$, then the control volume of the $u$-component is defined as $\mathbf{V}_{i+1 / 2, j}=\left[x_{i-1 / 2}, x_{i+1 / 2}\right] \times\left[y_{j-1}, y_{j}\right]$. Similarly, the control volume $\mathbf{V}_{i, j+1 / 2}$ for $v$ (velocity component in $y$-direction) is staggered up by half a grid. Staggered storage of the variables helps avoiding unphysical pressure fields, which might result from the pressure-velocity coupling step in solving the Navier-Stokes equations [13]. In the 3D case the scalar variables, such as pressure, are stored in the middle of the grid cell, while the velocity components are stored in the center of the corresponding face of the grid cell.

Discretization of the Navier-Stokes equations requires approximation of the differential operators appearing in the equations. The skew-symmetry of the differential operators $(\mathbf{u} \cdot \nabla)$ and $\nabla$ implies that the total energy of the flow is conserved when the flow is inviscid. It only decreases when there is dissipation. To achieve this on the discrete level a discrete skew-symmetric approximation of the operator $(\mathbf{u} \cdot \nabla)$ and a positive-definite approximation of $-\nabla \cdot \nabla$ are developed in Verstappen and Veldman [56]. Below we briefly give the main steps of the derivation of the method for a uniform Cartesian mesh employed here. This method also applies to non-uniform meshes.

The evolution of the total energy $(\mathbf{u}, \mathbf{u})=\int_{\Omega_{f}}(\mathbf{u} \cdot \mathbf{u}) \mathrm{d} V$ is given by:

$$
\begin{aligned}
\frac{\partial}{\partial t}(\mathbf{u}, \mathbf{u})= & \left(\frac{\partial \mathbf{u}}{\partial t}, \mathbf{u}\right)+\left(\mathbf{u}, \frac{\partial \mathbf{u}}{\partial t}\right) \\
= & -((\mathbf{u} \cdot \nabla) \mathbf{u}, \mathbf{u})-(\mathbf{u},(\mathbf{u} \cdot \nabla) \mathbf{u}) \\
& +\frac{1}{R e}((\nabla \cdot \nabla \mathbf{u}, \mathbf{u})+(\mathbf{u}, \nabla \cdot \nabla \mathbf{u}))-\left(\frac{\nabla p}{\rho_{f}}, \mathbf{u}\right)-\left(\mathbf{u}, \frac{\nabla p}{\rho_{f}}\right)
\end{aligned}
$$

where we substituted $\frac{\partial \mathbf{u}}{\partial t}$ from the Navier-Stokes equations. This expression reduces to

$$
\frac{\partial}{\partial t}(\mathbf{u}, \mathbf{u})=-\frac{2}{R e}(\nabla \mathbf{u}, \nabla \mathbf{u}) \leq 0
$$


if one takes into account (while transforming volume integrals into surface integrals) the skew-symmetry property of the differential operators:

$$
(\mathbf{u} \cdot \nabla)^{*}=-(\mathbf{u} \cdot \nabla) \text { and } \nabla^{*}=-\nabla
$$

where $^{(*)}$ stands for the adjoint operator [28]. Condition (2.6) also holds true on the discrete level, provided that the discrete approximations of differential operators inherit the (skew-)symmetry property of the continuous operators, given in (2.7). This yields an unconditionally stable and conservative spatial discretization scheme for the Navier-Stokes equations.

The discrete system of non-linear equations, obtained from integration of the Navier-Stokes and the continuity equations over the staggered control volumes, is given by:

$$
\begin{aligned}
\mathbf{V} \frac{\mathrm{d} \mathbf{u}_{h}}{\mathrm{~d} t}+\mathbf{C}\left(\mathbf{u}_{h}\right) \mathbf{u}_{h}+\mathbf{D} \mathbf{u}_{h}-\mathbf{M}^{*} \mathbf{p}_{h} & =0 \\
\mathbf{M} \mathbf{u}_{h} & =0
\end{aligned}
$$

with $\mathbf{u}_{h}\left(\mathbf{p}_{h}\right)$ being the vector of discrete velocities (pressure). For the definitions of the matrices and a detailed derivation, we refer to [56].

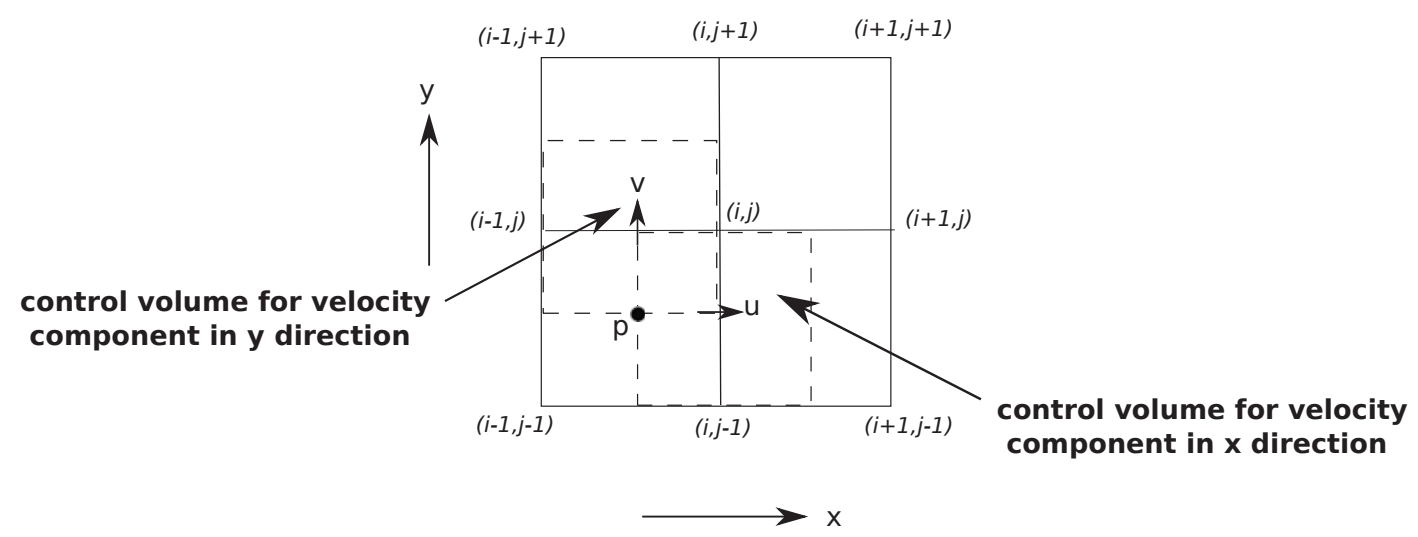

Figure 2-2: Control volumes for velocities in $x$ and $y$ directions in 2D

An important feature of the applied IB method is that meshing is done for the entire computational domain, including the areas occupied by the solid obstacles. The interface is formed by the faces of the basic grid cells. The phase indicator function $\Gamma$ is defined according to the material (solid or fluid) at the center of each basic cell. 
This indicates whether a given cell is in the solid $(\Gamma=1)$ or in the fluid region $(\Gamma=0)$ (Figure 2-3). The indicator function can easily be employed to determine whether a particle is deposited, i.e., hit or entered the solid domain. The advantage of this approach is that it is a local method and no wall-distances have to be computed which can be particularly challenging in an irregular 3D geometry.

For an efficient implementation of the method, we define $\Gamma^{f}, \Gamma^{e}, \Gamma^{v}$ on the faces, edges and vertices of a grid cell, respectively. For this we use the values of $\Gamma$ of the grid cells adjacent to a given face (edge, vertex). If one of the cells intersecting with a given face (edge, vertex) is solid then the face (edge, vertex) is also considered to be part of the solid. If we denote with $\Gamma(i, j, k)$ the value of the phase indicator function for the basic $(i, j, k)$ cell, then the value of $\Gamma^{f}$ on the face $\left(x_{i} \times\left[y_{j-1}, y_{j}\right] \times\left[z_{k-1}, z_{k}\right]\right)$ is denoted by $\Gamma_{i}^{f}(j, k)$ and is defined as:

$$
\Gamma_{i}^{f}(j, k)=\max \{\Gamma(i, j, k), \Gamma(i+1, j, k)\}
$$

For edges $\left(x_{i} \times y_{j} \times\left[z_{k-1}, z_{k}\right]\right)$ and $\left(x_{i} \times\left[y_{j-1}, y_{j}\right] \times z_{k}\right)$ the values of $\Gamma^{e}$ are denoted by $\Gamma_{i, j}^{e}$ and $\Gamma_{i, k}^{e}$, correspondingly, and defined as follows:

$$
\begin{aligned}
& \Gamma_{i, j}^{e}(k)=\max \{\Gamma(i, j, k), \Gamma(i+1, j, k), \Gamma(i, j+1, k), \Gamma(i+1, j+1, k)\} \\
& \Gamma_{i, k}^{e}(j)=\max \{\Gamma(i, j, k), \Gamma(i+1, j, k), \Gamma(i, j, k+1), \Gamma(i+1, j, k+1)\}
\end{aligned}
$$

And finally, for a vertex $\left(x_{i}, y_{j}, z_{k}\right)$ the value of $\Gamma^{v}$ is defined as:

$$
\begin{aligned}
\Gamma^{v}(i, j, k)= & \max \{\Gamma(i, j, k), \Gamma(i+1, j, k), \\
& \Gamma(i, j, k+1), \Gamma(i+1, j, k+1), \\
& \Gamma(i, j+1, k), \Gamma(i+1, j+1, k), \\
& \Gamma(i, j+1, k+1), \Gamma(i+1, j+1, k+1)\}
\end{aligned}
$$

In a similar manner face, edge, vertex phase-indicator functions can be defined for the rest of the faces, edges and vertices of the grid cells. These functions will be used when interpolating the flow field at the particle position. This is crucial for a physically consistent treatment of the particle tracking in a velocity field obtained 
with the IB method, i.e., including a small residual velocity where solid-fluid interfaces are located.

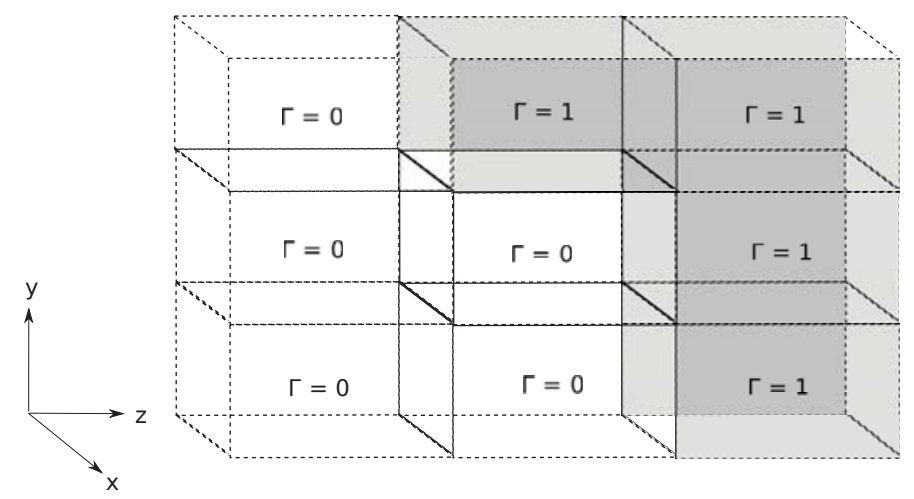

Figure 2-3: Example of representation of a solid obstacle using a phase-indicating function $\Gamma$, defined according to the material found at center of each basic grid cell. For grid cells that form the solid obstacle $\Gamma=1$, and in the part of the domain occupied by the fluid $\Gamma=0$.

\subsubsection{Particle tracking}

In the previous subsection we presented a numerical method for solving the governing equations for the gas phase. Here, we turn attention to solving the equations of motion for the particle phase, assuming steady flow for convenience. This assumption is not a principal limitation of the method and extension to time-dependent flow is readily made. The trajectory of an individual particle flowing in the gas is then computed from (2.3), using Euler's time-stepping method. We adopt a mixed formulation using implicit (for $\mathbf{v}$ ) and explicit (for $\mathbf{x}$ ) first order time integration methods:

$$
\left\{\begin{array}{l}
\mathbf{x}^{n+1}=\mathbf{x}^{n}+\Delta t \mathbf{v}^{n} \\
\mathbf{v}^{n+1}=\frac{S t}{S t+\Delta t}\left(\mathbf{v}^{n}+\frac{\Delta t}{S t} \mathbf{u}^{n}\right)
\end{array}\right.
$$

where $\mathbf{x}^{n}$ and $\mathbf{v}^{n}$ are the position and the velocity of the particle at time $t=t^{n}=n \Delta t$ and $\mathbf{u}^{n}$ is the gas velocity at the corresponding particle position (for simplicity, we will drop the subscript ' $h$ ' when referring to the discrete solution of (2.8)). Using the implicit scheme for the velocity allows one to consider small values of $S t$, without any restriction on the time step. For instance, in case of the explicit Euler method for the 
velocity the condition $\Delta t \leq 2 S t$ has to be satisfied to assure stability. This condition implies very small time steps for low values of the Stokes number.

In order to propagate the particle position and velocity over a time step the velocity of the gas at the particle position has to be computed. This requires that the flow velocity has to be interpolated at the particle position, from the gas velocity field $u$ at time $t^{n}$ and location $\mathrm{x}^{n}$. A simple choice for the interpolation method would be a trilinear interpolation. In the following subsection, we will first illustrate on an example in a one-dimensional (1D) representation that near the solid-fluid interface ordinary linear interpolation can cause a slight numerical inconsistency, which would imply numerical filtration of massless particles. A computational algorithm to avoid this inconsistency will be described and analyzed in 1D and extended to 3D subsequently.

\subsubsection{Analysis of particle motion near a solid-fluid interface in $1 \mathrm{D}$}

We will look at the motion of a particle in a linear flow field in 1D. Collision of a particle with a wall is determined by the velocity component normal to that wall. For the analysis of collision in the 3D linear flow field in the grid cell adjacent to a wall it is sufficient to analyze the 1D problem associated with the normal velocity separately. In this section we will show that a linear flow field that has a residual velocity at a solid-fluid interface may cause a collision of the particle with the wall even if it is massless. Such a situation closely corresponds to the actual velocity field computed by using a volume-penalizing IB method for the incompressible gas flow. This is unacceptable for a massless particle moving in a physically realizable velocity field and will also affect the computed filtration efficiency for inertial particles at low St. To avoid this, the linear velocity field can be corrected to remove the residual velocity at the solid-fluid interface.

Let us consider a test particle, that has no inertia and which moves with the flow from an initial position at $x_{0} \in[0, h], h>0$. Assume $x=0$ defines an interface 
between a liquid region $(0, h]$ and a solid region $x \in[-h, 0]$. The discrete values of the flow field $u$ are known at $x=0$ and $x= \pm h$. We will look at the set of cases corresponding to all possible situations when a particle is in a velocity field that moves it either away from the wall or toward the wall. These cases are illustrated in Figure 2-4: $(u(h)>0, u(0)>0),(u(h)>0, u(0)<0),(u(h)<0, u(0)>0)$ and $(u(h)<0, u(0)<0)$, where $|u(h)| \geq|u(0)|$. The velocity $u\left(x_{p}\right)$ at the particle location $x=x_{p}(t), x_{p}(t) \in[0, h]$, can be computed based on the given values $u(0)$ and $u(h)$. Let us now assume that $u(0) \neq 0$. In terms of the flow field this would mean that there is a residual velocity at the solid-fluid interface. Such a velocity field for a massless particle, i.e., a particle with $S t=0$, may lead to a collision with the wall. For instance, this may be the case for $u(h)<0, u(0)<0$. If we instead assume that at the interface the flow velocity is zero, then the corresponding linearly interpolated velocity $u\left(x_{p}\right)$ will be such that a massless particle will not be able to reach the wall. This can be seen on an example of the corresponding continuous problem that we look into next.

Consider the following initial value problem in $1 \mathrm{D}$, which can be derived from the equations of motion, taking into account the initial conditions:

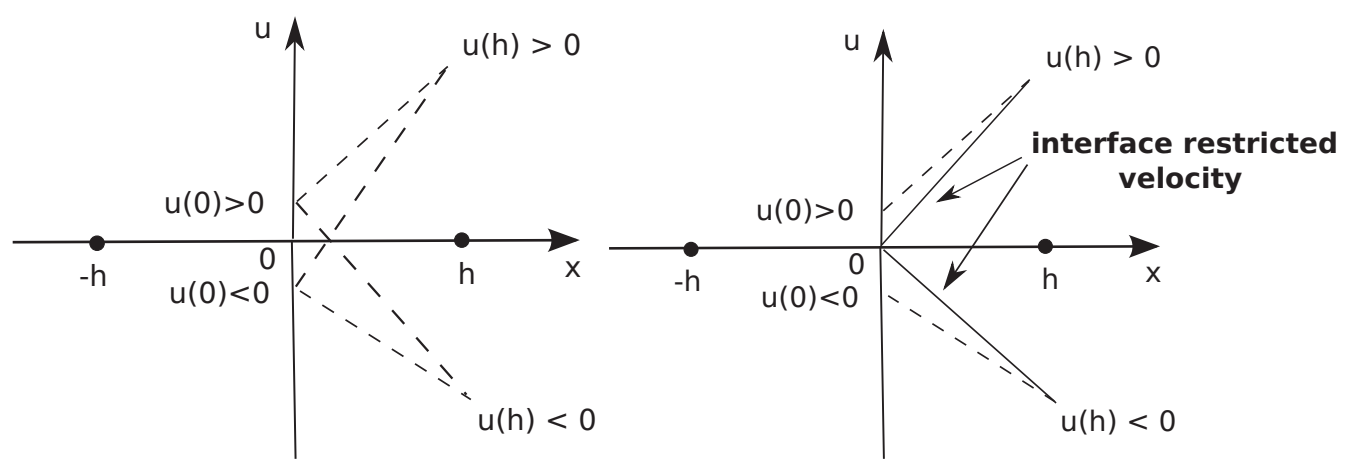

Figure 2-4: Linear interpolation of velocity field close to the solid-fluid interface: (a) linearly interpolated velocity when $u(0) \neq 0$, (b) interface restricted velocity interpolation, when $u(0)$ is set to zero. 


$$
\left\{\begin{aligned}
x^{\prime \prime}(t)+\frac{1}{S t} x^{\prime}(t)-\frac{u_{l}(x)}{S t} & =0 \\
x(0) & =x_{0} ; \quad x_{0} \in[0, h] \\
x^{\prime}(0) & =v_{0} \\
u_{l}(x) & =a x+b ; \quad a, b \in \mathbb{R}
\end{aligned}\right.
$$

The linear function $u_{l}$ can be seen as the linear interpolation of $u(h)$ and $u(0)$. Dynamics of this system depends on the initial conditions and the parameters $S t, a, b$. The analytical solution for this problem can be evaluated. For any $a$ and any initial velocity $v_{0}$, the particle's location $x$ will be positive, implying that the particle will not hit the wall. This can be seen if we look at the general solution, given by:

$$
x(t)=C_{1} \exp \left(\lambda_{1} t\right)+C_{2} \exp \left(\lambda_{2} t\right)-\frac{b}{a}
$$

with $\lambda_{1,2}=\frac{1}{2 S t}(-1 \pm \sqrt{1+4 a S t})$ and $C_{1}, C_{2} \in \mathbb{R}$, which can be computed from the initial conditions. In particular, it is interesting to study the behavior of the system for the limiting case of $S t \rightarrow 0$. Using the Taylor expansion of $\sqrt{1+4 a S t}$ in the expressions for $\lambda_{1}$ and $\lambda_{2}$, for the limit of $S t \rightarrow 0$ we can write $x(t)$ as:

$$
x(t)=C_{1} \exp (-t / S t)+C_{2} \exp (a t)-\frac{b}{a}
$$

If $b=0$, one can show that $x(t) \rightarrow x_{0} \exp (a t)>0$, as $S t \rightarrow 0$, hence showing the absence of a collision of a massless particle with the solid-fluid interface for all times. It can also be shown that if $a>0, b>0$, the particle position $x(t)>0$, for all times. If $a<0, b<0$ then $x(t) \rightarrow-b / a<0$, for $t \rightarrow \infty$, implying a finite time collision, even as $S t \rightarrow 0$. For $a b<0$ the analysis is more technical and depends on the relative values of $x_{0}, a$ and $b$. The main conclusion is that if $b=0$, no collision of a massless particle with a wall can occur.

One can show that the same holds true for the numerical solution, for instance calculated using Euler's explicit time integration method. It should be mentioned that $b=0$ is not a necessary condition, but a sufficient one. In terms of our 1D 
setting this means that if $u(0)=0$, without any restriction on $u(h)$, a collision of a massless particle with the wall will not arise. To illustrate the four cases identified above, in Figure 2-5 we include $(a=1, b=1),(a=1, b=0),(a=-1, b=-1)$ and $(a=-1, b=0)$. We compute the 'arrival time' at which the trajectory of an inertial particle with initial condition $(x(0), v(0))=(1,-1)$ and Stokes number $S t$ crosses the line $x=0$. The arrival time is estimated by determining the time $t$ for which $x(t)=0$. The value of the arrival time is calculated through linear interpolation of two consecutive particle positions at times $t^{n}$ and $t^{n+1}$, such that $x^{n}>0$ and $x^{n+1} \leq 0$. We see in the figure that it takes a finite time for a massless particle to hit the wall for the velocity field defined by $a=-1, b=-1$. The other three curves do confirm the analysis made based on the signs of $a$ and $b$ : particles with a small Stokes number do not arrive at $x=0$ in finite time.

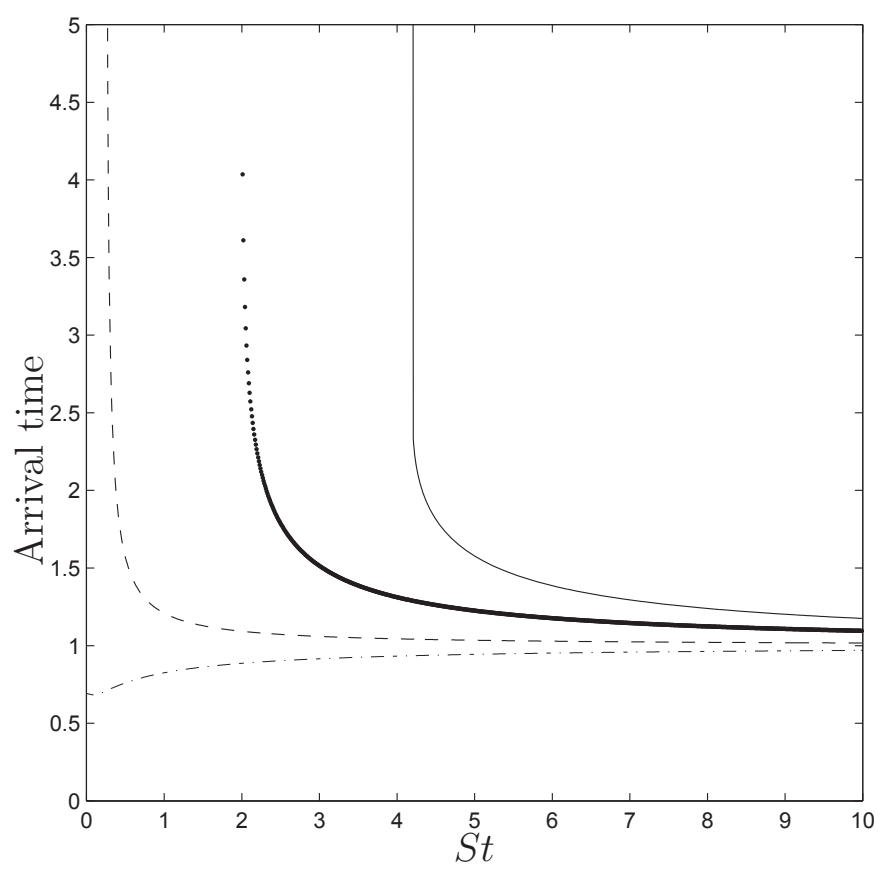

Figure 2-5: Arrival time versus the Stokes number for a particle moving in a linear velocity field $u_{l}=a x+b$. Four different velocity fields are considered: $a=1, b=1$ ( solid); $a=1, b=0$ (dot); $a=-1, b=-1$ (dash dot); $a=-1, b=0$ (dash).

To ensure that massless particles will not enter a solid part of the domain, it is necessary to restrict the interpolation of the velocity field at the particle position in such a way that at the wall the interpolated velocity is zero. Therefore, the 
interpolated velocity field is then defined $u_{l}(x)=(u(h) / h) x$. The main steps in the 1D setting are: (i) localization of the particle, (ii) determine whether the particle is in a grid cell that is adjacent to a solid-fluid interface, (iii) produce a linear velocity field in the grid cell that is consistent with the no-slip condition at a solid wall. Based on this simple algorithm, in the following subsection we describe the interpolation of the gas velocity close to the solid-fluid interface in $3 \mathrm{D}$ such that the physically consistent tracking of massless particles is maintained.

\subsubsection{D solid-fluid interface velocity interpolation}

As we have seen in the 1D example, massless particles will not be captured at the solid, provided that the interpolated value of the gas velocity at the solid surface is zero. In the fluid simulations the velocities at the interface are of order $10^{-5}$ related to the damping parameter $\epsilon$. Based on the discrete phase-indicator function $\Gamma$, the computed velocity can be mapped onto the grid in such a way that prior to the interpolation of the fluid velocity at the particle position at all solid-fluid interfaces the velocities are zero. This only affects the velocity field seen by the particles and is not adopted as correction for the IB-computed velocity field itself. To implement this, auxiliary values of the velocity fields are computed, defined at the edges and vertices of the basic grid cells. These values are evaluated by interpolating the velocity $\mathbf{u}$ to the edges and vertices, taking into account whether a given face belongs to a solidfluid interface or not. Setting the introduced auxiliary velocities to zero once they lie on a solid-fluid interface is the key step in our computational technique for avoiding deposition of massless particles.

As an example we consider the $x$-component of the velocity vector $\mathbf{u}$. Let us denote $\Delta x_{i}=x_{i+1}-x_{i}, \Delta y_{j}=y_{j+1}-y_{j}$ and $\Delta z_{k}=z_{k+1}-z_{k}$. Particle localization determines which points need to be included in the interpolation. Let us assume that the particle at time $t$ is located at $\mathbf{x}_{p}=\left(x_{p}, y_{p}, z_{p}\right) \in \mathbb{R}^{3}$, where

$$
x_{i-1} \leq x_{p} \leq x_{i} ; \quad y_{j-1}-\frac{\Delta y_{j}}{2} \leq y_{p} \leq y_{j}-\frac{\Delta y_{j}}{2} ; \quad z_{k-1}-\frac{\Delta z_{k}}{2} \leq z_{p} \leq z_{k}-\frac{\Delta z_{k}}{2} .
$$




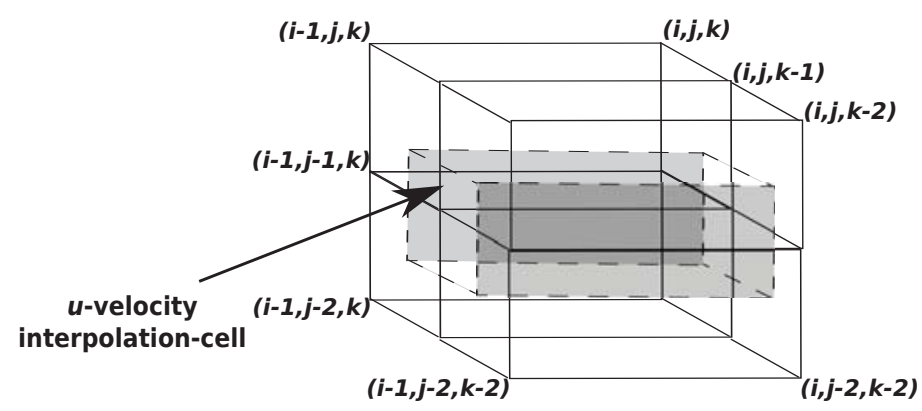

Figure 2-6: Velocity interpolation-cell in 3D for the velocity component in $x$ direction.
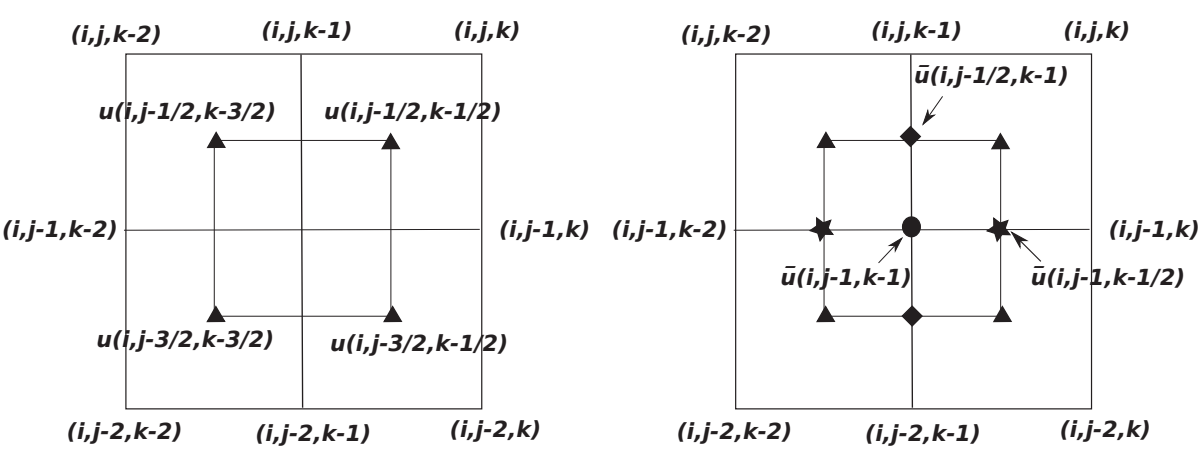

Figure 2-7: (a) Velocities defined at points marked by $\boldsymbol{\Delta}$ are available from the IB. (b) Velocities defined at points marked by $\star, \downarrow$ and $\bullet$ are the auxiliary, interpolated velocities.

At the particle location $\left(x_{p}, y_{p}, z_{p}\right)$ the gas velocity $u\left(\mathbf{x}_{p}(t), t\right)$ is computed by interpolating auxiliary velocities at the faces of the $u$-velocity interpolation-cell, as shown in Figure 2-6. The $u$-velocity interpolation-cell is shifted half a grid cell from the basic grid in the $y$ and $z$ directions. Here we use fractional index notation to denote the velocities. For instance, $u\left(i, j-\frac{1}{2}, k-\frac{1}{2}\right)$ represents the velocity at $\left(x_{i}, y_{j}-\right.$ $\left.\frac{\Delta y_{j}}{2}, z_{k}-\frac{\Delta z_{k}}{2}\right)$, as shown in Figure 2-7(a). The main step is setting the auxiliary values and IB resolved velocities to zero once the points where the velocities are defined lie on the solid-fluid interface. To do this we use the values of previously defined $\Gamma^{f}, \Gamma^{e}$ and $\Gamma^{v}$. This defines the auxiliary velocities $\bar{u}$ as follows: 


$$
\begin{aligned}
\bar{u}\left(i, j-\frac{1}{2}, k-\frac{1}{2}\right) & =u\left(i, j-\frac{1}{2}, k-\frac{1}{2}\right)\left(1-\Gamma_{i}^{f}(j, k)\right) \\
\bar{u}\left(i, j-\frac{1}{2}, k-1\right) & =\frac{1}{2}\left[u\left(i, j-\frac{1}{2}, k-\frac{1}{2}\right)+u\left(i, j-\frac{1}{2}, k-\frac{3}{2}\right)\right]\left(1-\Gamma_{i, k-1}^{e}(j)\right) \\
\bar{u}\left(i, j-1, k-\frac{1}{2}\right) & =\frac{1}{2}\left[u\left(i, j-\frac{1}{2}, k-\frac{1}{2}\right)+u\left(i, j-\frac{3}{2}, k-\frac{1}{2}\right)\right]\left(1-\Gamma_{i, j-1}^{e}(k)\right) \\
\bar{u}(i, j, k) & =\frac{1}{4}\left[u\left(i, j-\frac{1}{2}, k-\frac{1}{2}\right)+u\left(i, j-\frac{1}{2}, k-\frac{3}{2}\right)\right. \\
& \left.+u\left(i, j-\frac{3}{2}, k-\frac{1}{2}\right)+u\left(i, j-\frac{3}{2}, k-\frac{3}{2}\right)\right]\left(1-\Gamma^{v}(i, j, k)\right)
\end{aligned}
$$

Within the velocity interpolation-cell auxiliary velocities are now available. This approach can be directly applied to the case of a non-uniform Cartesian mesh since all operations are defined in index-space; these transfer directly to physical space.

Having the values of the velocity field at the vertices and edges of the grid cells, the particle localization is reduced to defining in which quarter of the interpolation-cell the particle is found. If we look at the $x=x_{i}$ face (cf. Figure 2-7(b)), such localization defines the location of the particle with respect to $\left(y_{j-1}, z_{k-1}\right)$. The interpolation of the velocity at the particle location is then done using the auxiliary values of the velocities, e.g., with a trilinear interpolation. Once the velocity field is computed this way at the particle location, the particle velocity and location at the next time step can be computed from (2.12). With this we can follow trajectories of the particles moving in a given velocity field.

In the following section we will present results obtained from numerical simulations based on the described algorithms.

\subsection{Impaction filtration of aerosol droplets in porous media}

One of the applications of particle tracking in a porous material is the prediction of particle deposition on a filter. In this section we turn to one of the filtration mechanisms, called impaction filtration. Two structured porous media were considered as a point of reference: these consist of a parallel in-line and staggered arrangement of 
square rods in 3D (cf. Figure 2-1). We focus on the computation of the filtration efficiency for these reference cases, to validate and establish the numerical method.

\subsubsection{The motion of particles in model porous media}

We show the motion of inertial particles and its relation with the inner structure of the porous medium. The detailed flow that develops will have a direct consequence on the deposition rate. To understand this, we first look at the flow field for both the in-line and the staggered geometries. In Figure 2-8 we present vector plots of velocity components in the $x$ and $y$ directions, calculated for $R e=100$ (cf. Lopez Penha et al [31]). The flow is computed by applying a pressure gradient in the $x$ direction with spatial resolutions $64 \times 64 \times 4$ (for the in-line geometry) and $128 \times 64 \times 4$ (for the staggered geometry). The sizes of the computational domains are $1 \times 1 \times 1$ and $2 \times 1 \times 1$ for the in-line and staggered geometries, respectively. In the in-line geometry a 'channel'-type flow pattern is formed between the upper and the lower layer of rods. The apparent channel flow in the in-line geometry is broken in case of a staggered arrangement of the rods. In both porous media formation of recirculation zones behind the solid walls is observed.

The structured flow that develops can be appreciated in more detail by visualizing the trajectories of particles moving in it as a function of a non-dimensional time $t$. The steady state fluid flow is precomputed and afterwards particles are introduced. We first consider structured initial positions to help developing the method. For the simulation of the filtration characteristics a different initial distribution of the particles is used. In fact, particles are introduced in the fluid domain at random locations, such that particles cover the fluid domain statistically evenly and no clustering of particles arises. The initial velocity of a particle is taken equal to the local velocity of the fluid. Periodic boundary conditions for the particle transport are applied in all directions. We take $10^{4}$ particles that are initially positioned on the line $x=0, z=0$ and simulate their motion. We concentrate first on motion through the staggered arrangement of rods. Two values of the Stokes number are considered, for which a qualitative difference in the trajectories through the porous medium is observed: $S t=0.01$ and 


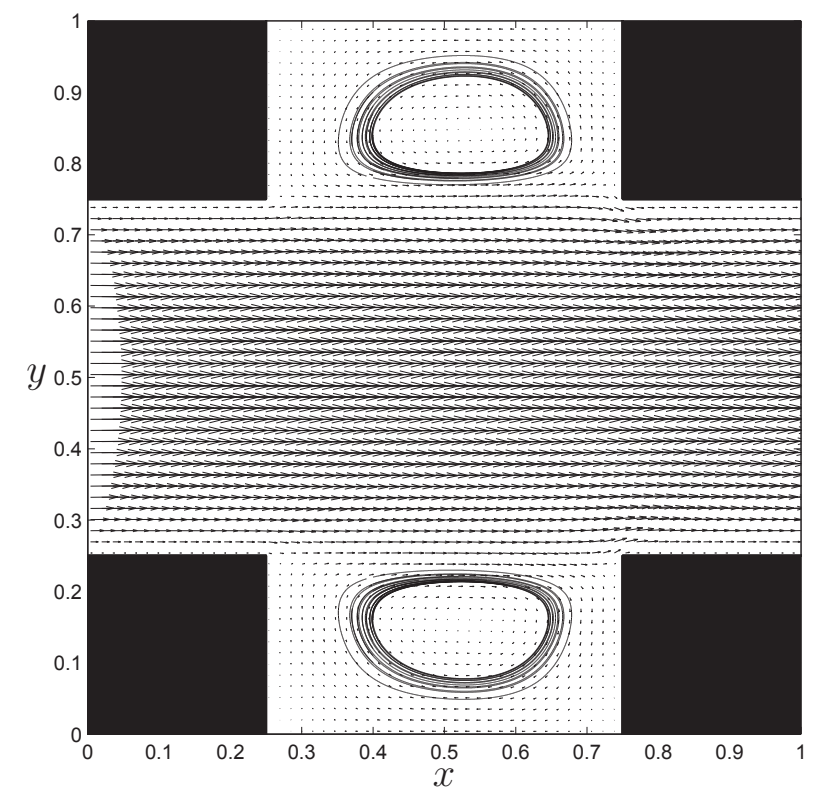

(a) In-line arrangement (resolution $64 \times 64 \times 4)$

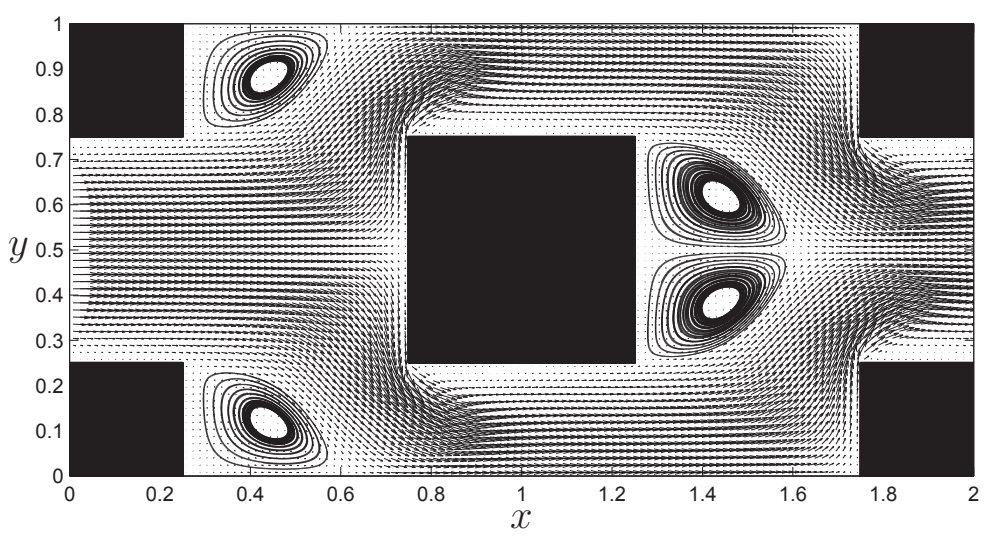

(b) Staggered arrangement (resolution $128 \times 64 \times 4$ )

Figure 2-8: Vector representation of flow field. Velocity components in the $x$ and $y$ directions are presented for $R e=100$. 
$S t=0.05$. These values of the Stokes number correspond approximately to a particle diameter of $16 \mu \mathrm{m}$ and $37 \mu \mathrm{m}$, respectively. The positions of non-deposited particles are registered at non-dimensional times $t=0.1, t=0.3, t=0.4, t=0.8, t=0.9$ and $t=1$, shown in Figure 2-9 for $S t=0.01$ and in Figure 2-10 for $S t=0.05$. For both Stokes numbers a fraction of the droplets gets captured by the upstream face of the solid rod in the center of the REV. In case $S t=0.01$, particles, that do not collide with the front face of the center rod, move around it without being captured. These leave the REV of the porous medium and enter on the opposite side, because of the periodic conditions, quite closely following streamlines that do not lead to (much) collision with the solid. On the other hand, particles with a slightly larger Stokes number $S t=0.05$ (this corresponds to a diameter that is approximately twice larger) behave qualitatively differently. Those that manage to avoid collision with the center rod, are to a large extent captured by the solid squares in the left and right corners. This example with structured initial conditions hints at a complex dependence of the motion and ultimately of the filtration efficiency on the Stokes number. To this we turn next.

We now consider $10^{4}$ particles that are initially positioned randomly in the fluid domain, using a linear congruential generator (LCG). In Figures 2-11 and 2-12 the location of a large ensemble of particles at $S t=1$ is presented for the in-line and the staggered geometries, respectively. Only suspended, i.e. non-deposited, particles are shown. For this reason, the number of particles shown in the figures is smaller than the initial number of suspended particles. In the sequel, we refer to traveled distance 'on average', as the distance that a particle would travel having the bulk velocity $U$. The positions of particles are registered (a) at an early stage in the simulation, (b) when particles on average have traveled through half of the REV and (c) when particles on average have traveled well across the REV. Snapshots of particle positions are taken at $t=0.05, t=1$ and $t=3$. For the in-line case, particles that are initially positioned in the wakes, behind rods, gradually move towards the walls in a form of 'centrifugal' motion and get captured. Particles in the central part, that effectively appears to form a channel, smoothly continue their journey without much structuring, e.g., by 


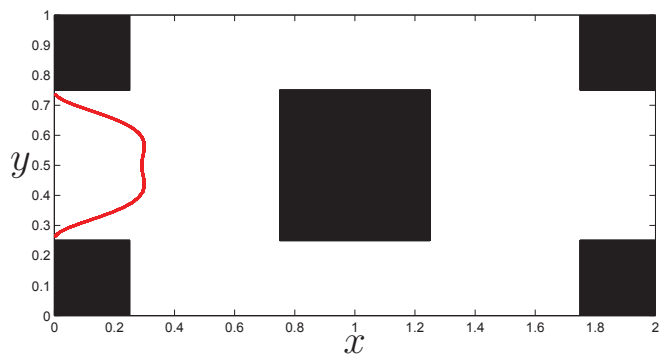

(a) $t=0.1$

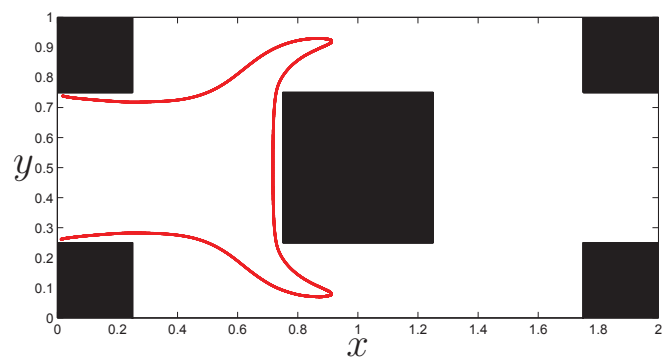

(c) $t=0.4$

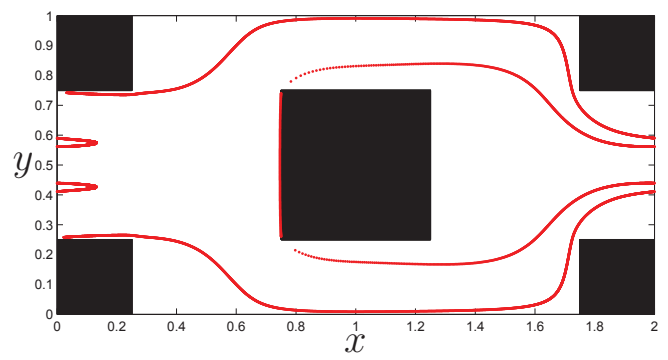

(e) $t=0.9$

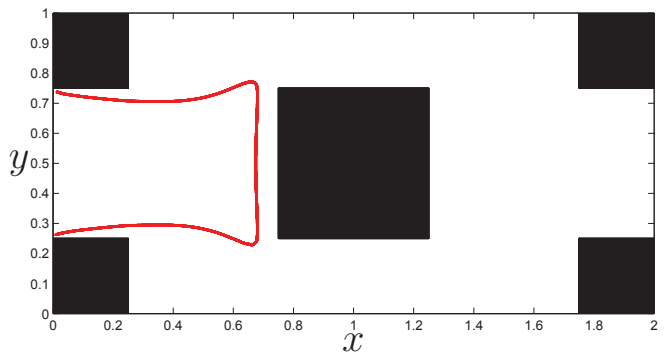

(b) $t=0.3$

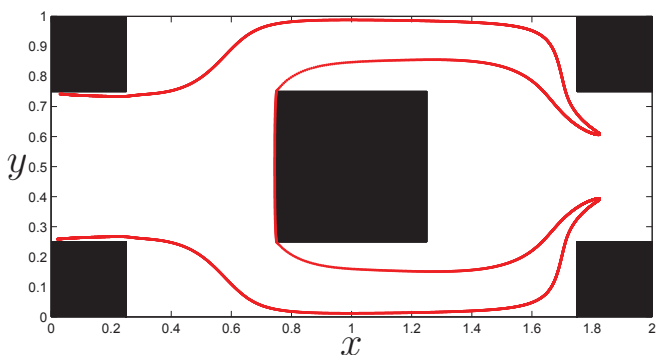

(d) $t=0.8$

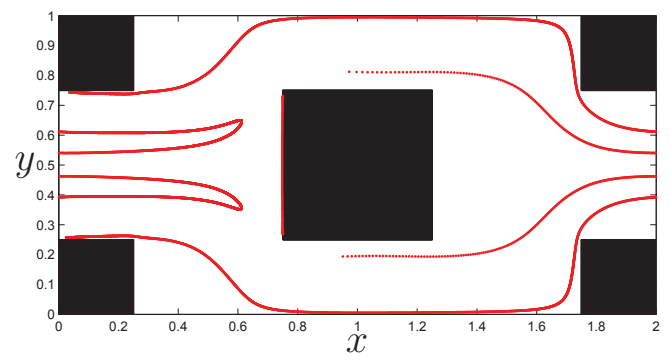

(f) $t=1$

Figure 2-9: Particle dynamics for $S t=0.01$ in the staggered geometry. Initially, $10^{4}$ particles are placed at the line $x=0, z=0$ of the fluid part of the domain. The initial velocity of the individual particles is taken equal to the fluid velocity at the particle position. The locations of non-deposited particles are registered at times $t=0.1, t=0.3, t=0.4, t=0.8, t=0.9$ and $t=1$. 


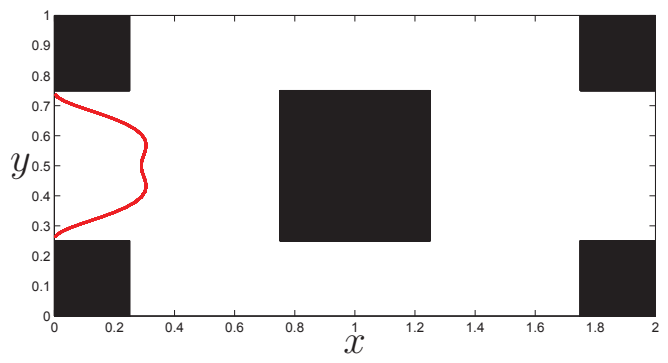

(a) $t=0.1$

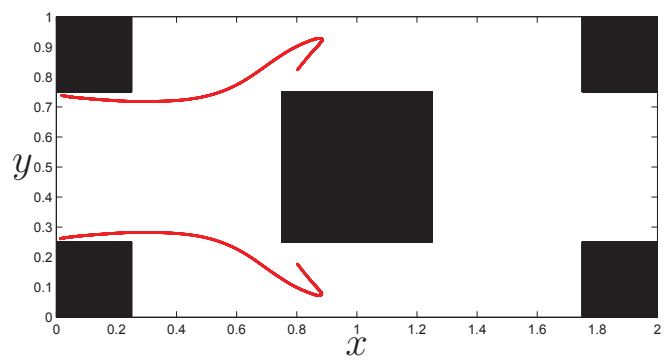

(c) $t=0.4$

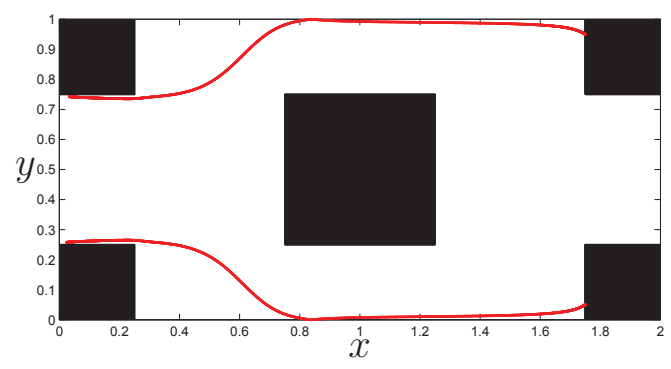

(e) $t=0.9$

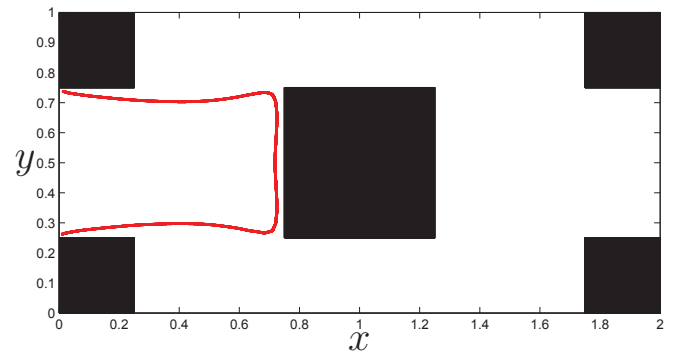

(b) $t=0.3$

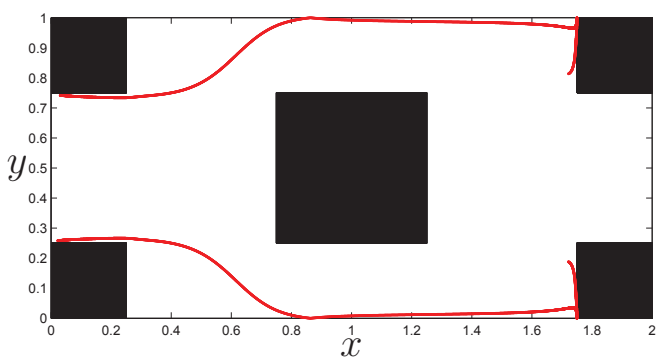

(d) $t=0.8$

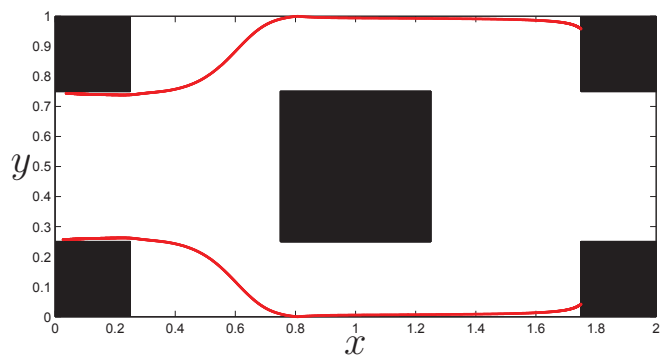

(f) $t=1$

Figure 2-10: Particle dynamics for $S t=0.05$ in the staggered geometry. Initially, $10^{4}$ particles are placed at the line $x=0, z=0$ of the fluid part of the domain. The initial velocity of the individual particles is taken equal to the fluid velocity at the particle position. The locations of non-deposited particles are registered at times $t=0.1, t=0.3, t=0.4, t=0.8, t=0.9$ and $t=1$. 
local clustering. The situation is different for the staggered geometry: depending on the Stokes number, particles form clusters near preferential paths, as presented in Figure 2-12.

Next, we turn to the effect of the Stokes number on the trapping of the particles by the flow streamlines. In Figures 2-13 and 2-14 we show particle positions for inline and staggered geometries for $S t=1, S t=0.1$ and $S t=0.01$. The snapshots are taken when particles have on average traveled twice through the REV, i.e., at $t=3.5$. For the in-line geometry the particles follow trajectories quite similar to what would apply in a channel flow - this holds for all Stokes numbers, i.e., the particles mainly follow straight-line trajectories parallel to the walls, with a small exception near the gaps between the rods. In this geometry, particles with an intermediate and large Stokes number, i.e., $S t=0.1$ and $S t=1$, that were initially positioned in the recirculation zones behind the rods, get gradually deposited at the solid material as a result of centrifugal motion developed by the flow streamlines. Particles with small Stokes number $S t=0.01$ get captured extremely slowly as they mainly follow the streamlines. In the staggered geometry, the presence of the central rod results in more rapid capturing of particles with intermediate and large Stokes numbers compared to the in-line geometry. Particles with $S t=1$ deviate from the fluid streamlines and gradually get captured by the solid. Even though particles with $S t=0.1$ are also rapidly captured, the deposition patterns for this Stokes number differ from the ones for $S t=1$. On the other hand, in the staggered geometry some part of the particles with $S t=0.01$ form a 'band', wrapping around the central rod and stay in this band, effectively without being captured for comparably long time. This is similar to the behavior observed in case of structured initial positions, presented in Figure 2-9. It will be shown further in this section that such structuring influences the filtration of particles in a manner that depends quite non-uniformly on the particle inertia.

\subsubsection{Impaction filtration of aerosol droplets in porous media}

In Section 2.4.1 we presented the qualitative differences of particle motion in the in-line and the staggered geometries. In this section, we turn our attention towards 


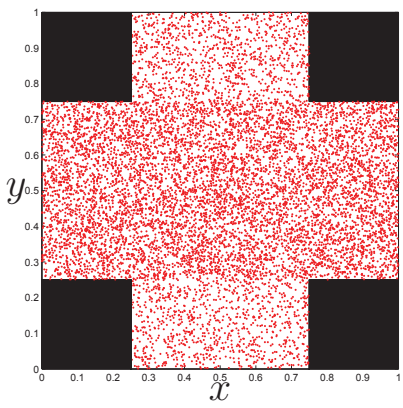

(a) $t=0.05$

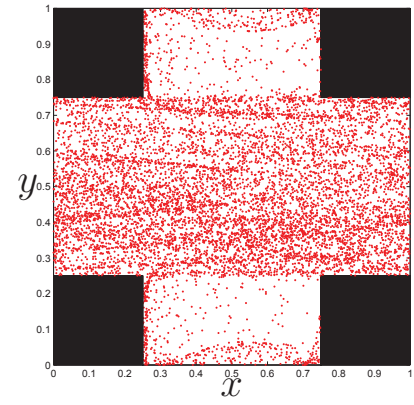

(b) $t=1$

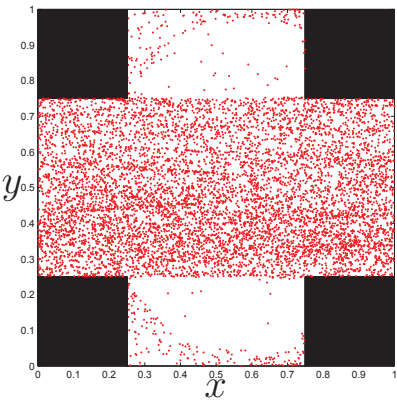

(c) $t=3$

Figure 2-11: Particle dynamics for $S t=1$ in the in-line geometry. Initially, $10^{4}$ particles are randomly distributed in the fluid part of the domain. Initial velocity of the individual particles is taken equal to the fluid velocity at the particle position. The locations of non-deposited particles are registered at times $t=0.05, t=1$ and $t=3$.

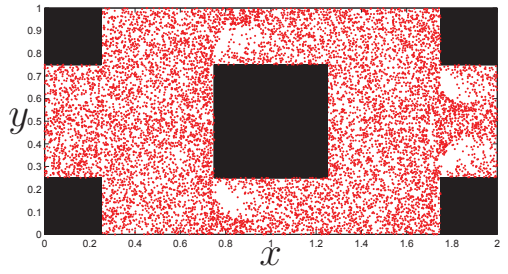

(a) $t=0.05$

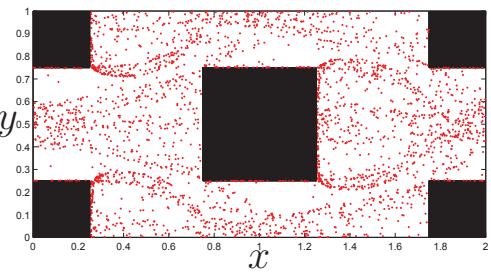

(b) $t=1$

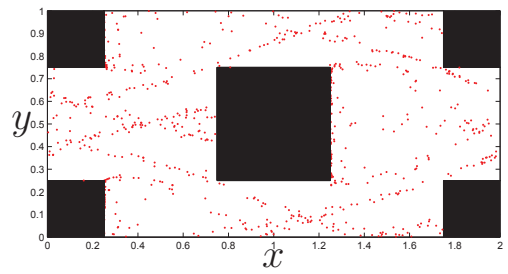

(c) $t=3$

Figure 2-12: Particle dynamics for $S t=1$ in the staggered geometry. Initially, $10^{4}$ particles are randomly distributed in the fluid part of the domain. Initial velocity of the individual particles is taken equal to the fluid velocity at the particle position. The locations of non-deposited particles are registered at times $t=0.05, t=1$ and $t=3$. 


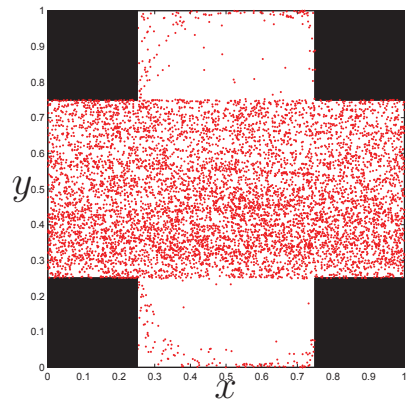

(a) $S t=1$

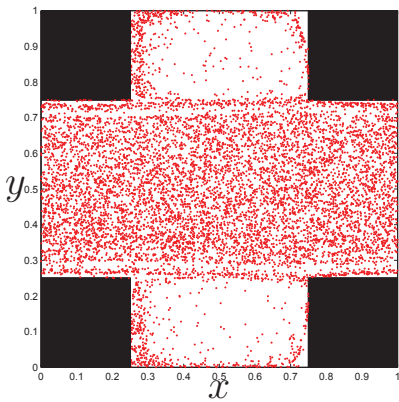

(b) $S t=0.1$

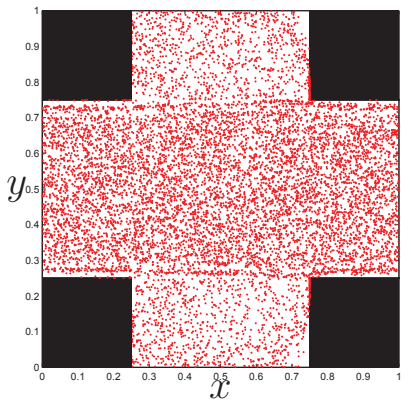

(c) $S t=0.01$

Figure 2-13: Distribution of non-deposited particles in the in-line geometry at $t=3.5$ for different values of $S t$.

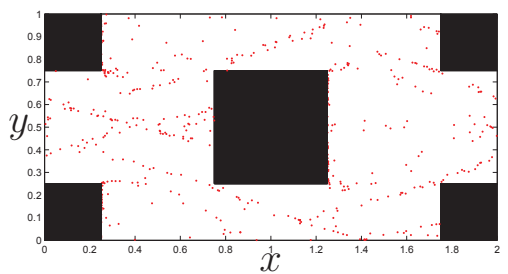

(a) $S t=1$

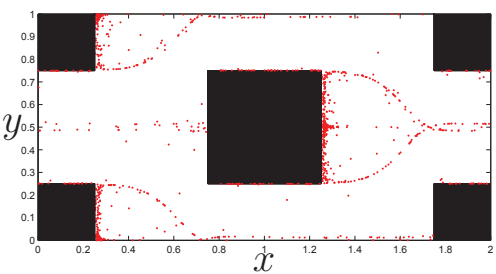

(b) $S t=0.1$

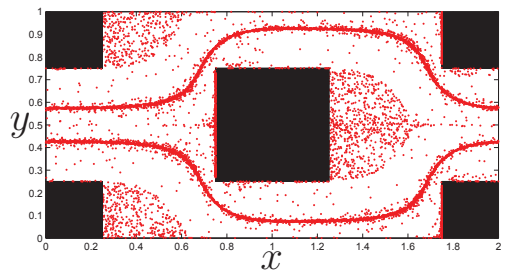

(c) $S t=0.01$

Figure 2-14: Distribution of non-deposited particles in the staggered geometry at $t=3.5$ for different values of $S t$. 
computations of particle deposition in these two distinct porous media geometries. Generally, the following steps must be taken into account for effective computations of the particle deposition on the surface of a porous filter:

- the geometry of the porous material through which particles travel must be specified,

- steady flow field simulations for the computation of the corresponding velocity field must be performed,

- initialization of particles' positions and their velocities at these positions must be carried out,

- simulations of particle trajectories suspended in the computed steady flow solution must be executed, and

- appropriate post-processing of data must be applied, e.g., computation of the so-called filtration efficiency function that characterizes deposition in time.

The so-called filtration efficiency function $E(t)$ is defined as the ratio between the number of not yet deposited particles that are still present in the flow $N(t)$ at time $t$ and the initial number of particles $N(0)$ :

$$
E(t)=\frac{N(t)}{N(0)}
$$

We distinguish between deposited and non-deposited particles using the masking function introduced in Section 2.3, i.e., a particle is considered as deposited at time $t$ if its position belongs to a grid cell marked by $\Gamma$ equal one.

In order to obtain statistically reliable results for the decay curve in time, defined by $E(t)$, aspects such as the initial number of particles and the number of different random initial particle positions were considered in detail. Here, we adopt a single configuration of $10^{4}$ particles which are randomly distributed in the whole fluid domain $\Omega_{f}$. Subsequently, initialization of particle positions and velocities interpolated 
from the mesh values to their actual position is performed. We take the local velocity of the fluid as the initial velocity of a given particle, which means that initially particles have zero acceleration. This results in a short transition (in the considered case of about $1 / 100$ of the time scale, i.e., 0.001 seconds) for a particle to adopt to the flow accordingly to its Stokes number.

The filtration efficiency is computed here in terms of the decay rate $\gamma$, defined conveniently as long-time decay $\gamma(S t)=1-E(T)$. Here, the time scale $T$ is larger compared to the flow-through time for the REV, i.e., the time needed for one passage through the domain at bulk velocity. We considered in this paper $T=10$, corresponding to about five passages of the REV, which was found to be representative and sufficient to collect required statistics. For a range of Stokes numbers it was established that by increasing the initial number of particles 100 times the effect on the long-time decay rates is of order $10^{-3}$. This motivates our choice of the $10^{4}$ initial number of particles, allowing statistically reliable computation of $\gamma$ as a function of Stokes number.

To assess the importance of proper numerical treatment for low Stokes numbers we considered the staggered alignment of rods. In this geometry filtration of introduced particles not only corresponds to the considered flow conditions, but strongly depends on the size of the particles. The point-like particles with negligible mass flow along the streamlines with zero filtration efficiency, while the particles with significant mass hit immediately the central element of the REV. In addition, the central element itself causes remarkable flow redirection that further affects the deposition. As a final result, the filtration efficiency sharply spans between zero and one depending on the Stokes number.

In Figure 2-15(a) the distribution of deposited particles without applying 'no-slip' consistent treatment for $S t=0$ is presented. For this Stokes number about $8 \%$ of particles is getting captured by the solid, while there should be no filtration as discussed in previous section. In Figure 2-15(b) we compare the decay rate $\gamma$ computed for two decay curves $E$, corresponding to velocity interpolation using auxiliary 'no-slip' consistent values and velocity interpolation based directly on the computed velocities 
from the immersed boundary method. As discussed in Section 3, the residual velocity at the solid-fluid interface resulting from the IB method affects the motion of very small particles close to a wall to such an extent, that numerical filtration dominates physical filtration. Larger particles 'sense' their inertia and their trajectories and filtration are not significantly affected by the residual velocity. For particles with $S t \lesssim 1 / 30$, the additional numerical filtration is larger than the actual physical filtration. Further on we present only results coming from proper numerical treatment of the filtration.

Next, we turn our attention to comparison of deposition in two considered REV geometries. As expected, qualitative and quantitative differences are observed in the decay curves for the two porous media (cf. Figure 2-16). In the in-line geometry(cf. Figure 2-16(a)) the filtration efficiency does not depend very strongly on the particle inertia since for the considered steady flow conditions the particles are flowing through the domain undisturbed by the geometry. This results in low filtration efficiency even for particles characterized by comparatively large Stokes numbers. In the staggered arrangement much stronger dependence between the filtration and Stokes number is observed. This can also be qualitatively observed in the patterns of particle trajectories presented as snapshots of particle positions in Figure 2-14. As expected, particles with negligible mass (small Stokes number) are not deposited since they flow along the streamlines. When particle mass becomes larger the inertial effects become dominant as well. The staggered geometry with an obstacle in the center of the REV, together with discussed flow structuring, induces significant growth of the filtration efficiency, as shown in Figure 2-16(b).

The decay rate $\gamma$ for two porous geometries is shown to illustrate the difference in filtration characteristic. For a range of Stokes numbers the dependence of $\gamma$ is presented in Figure 2-17. For the in-line geometry $\gamma$ increases uniformly for $S t \lesssim 0.2$. For higher Stokes numbers, up to about 0.4, the filtration is less effective, while a region of increased $\gamma$ is found after a sharp jump in the filtration efficiency around $S t=0.4$. In the staggered arrangement the dependence of $\gamma$ on $S t$ is more complex. In Figure 2-17 we have included all data obtained with the no-slip consistent algorithm, 


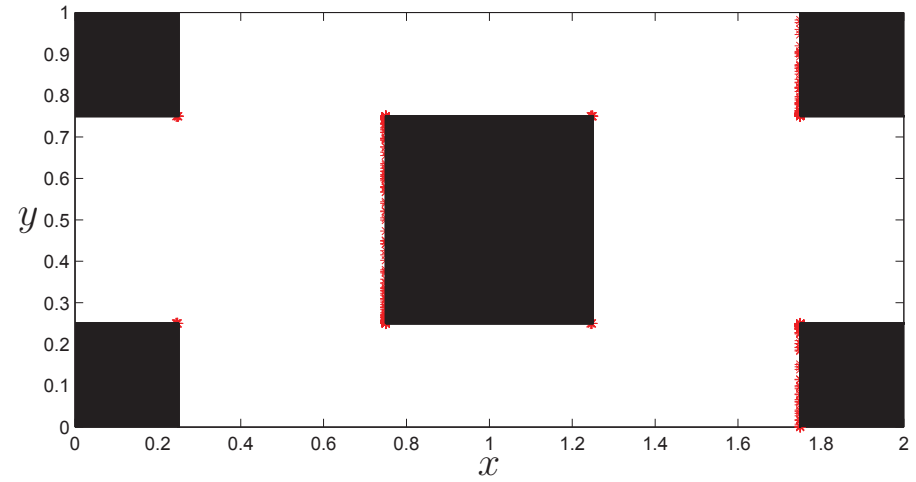

(a)

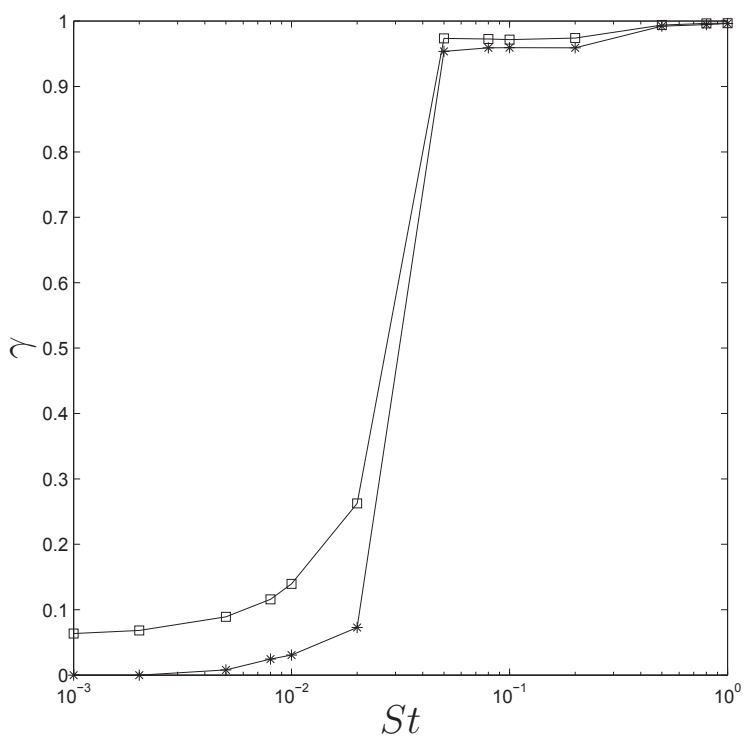

(b)

Figure 2-15: (a) Distribution of deposited particles without 'no-slip' consistent treatment for $S t=0$. (b) Effect of 'no-slip' consistent treatment in the staggered geometry: decay rate as a function of Stokes number with (star) and without (square) consistent 'no-slip' treatment near the solid-fluid interface. 


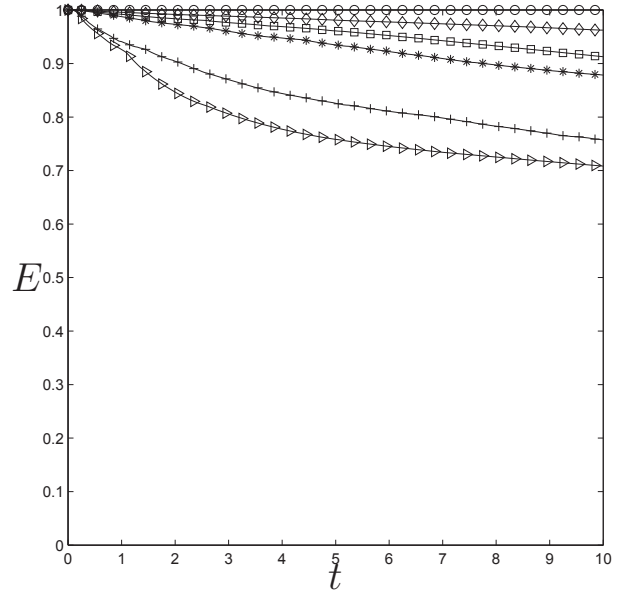

(a) In-line geometry.

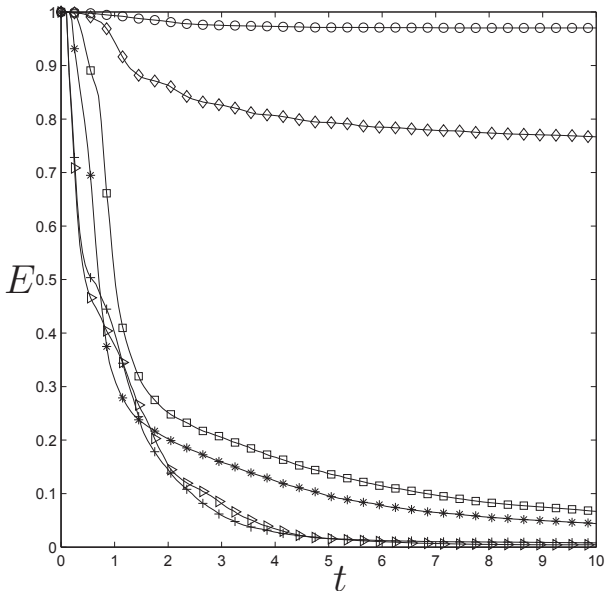

(b) Staggered geometry.

Figure 2-16: Decay of number of particles suspended in the flow as a function of time for different values of the particle Stokes numbers: $S t=0.01$ (circle), $S t=$ 0.03 (diamond), $S t=0.04$ (square), $S t=0.05$ (star), $S t=0.5$ (plus) and $S t=1$ (triangle).

as already shown in Figure 2-15(b). In addition, we included results from further variations in the Stokes number, displaying more detail in the filtration characteristic for this structured porous medium. We may distinguish two regimes: $S t<0.04$ and $S t \geq 0.04$. In the first regime $\gamma$ is rather small, corresponding to particles that are agile enough to move around the center rod in the REV. Particles with $S t \geq 0.04$ are filtered rapidly, a consequence of the motion as illustrated in Figure 2-10 at $S t=0.05$. For these Stokes numbers around $90 \%$ of the particles is captured by $t=10$. The additional complexity of the dependence of $\gamma$ on $S t$ for the staggered arrangement is illustrated, e.g., by the behavior for $0.03<S t<0.04$ and further non-uniform growth of $\gamma$ around $S t \approx 0.4$. These features can be reliably extracted using the new simulation method. Values of $\gamma$ as a function of Stokes number are statistically reliable, implying that the complex structure observed in both geometries are signs of the actual inertial behavior of particles within the porous media. Inclusion of random Brownian effects on the motion of the droplets will influence the detailed structure of the filtration characteristics at different St. However, the main features and filtration regimes as observed in Figure 2-17 are expected to remain, particularly for the higher 


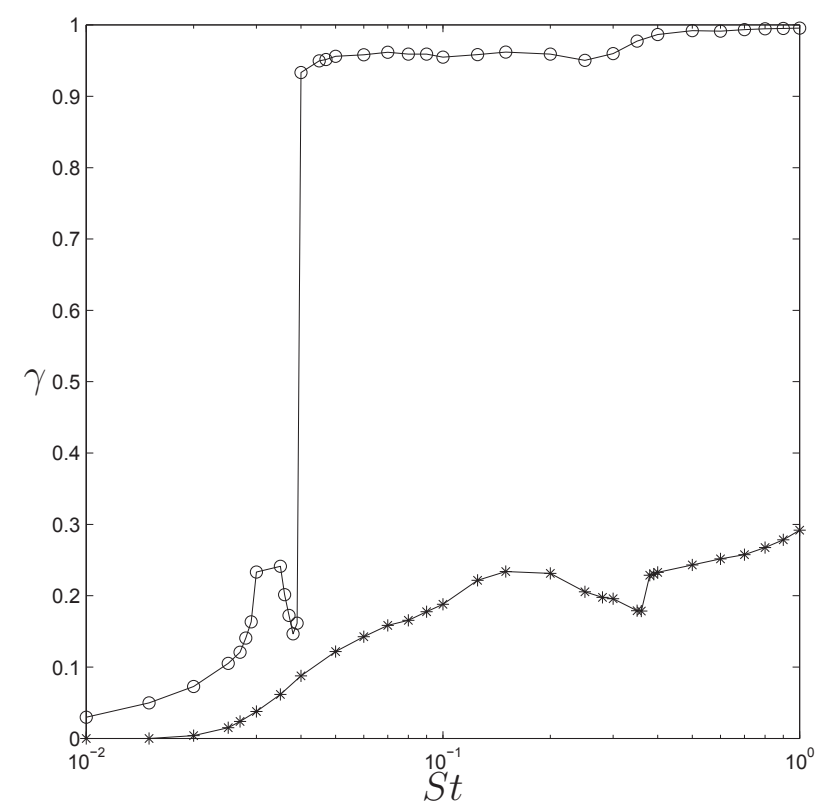

Figure 2-17: Decay rate $\gamma$ as a function of Stokes number. Data points marked with a star correspond to the in-line arrangement, and circles represent the data points for the staggered arrangement. The standard deviation of data points from the mean value averaged over 100 different initial random distributions is of order $10^{-3}$.

$S t$ values. The precise effects of Brownian motion can be investigated on the basis of the current 'no-slip consistent' approach, which is the subject of the following chapter.

\subsection{Conclusions}

We proposed a particle tracking method to study filtration properties of various porous filters. We focused our attention on particle deposition on the solid filter surface due to inertial impaction. A numerical approach was described to simulate the motion of a large number of particles suspended in a gas flow. The new computational method avoids numerical filtration of massless particles. We considered two structured porous media in 3D, composed of in-line and staggered arrangements of square rods. It was established that in the staggered geometry filtration efficiency computed without 'no-slip' correction of the velocities leads to considerable systematic error in case of very small particles. This shows that the developed numerical 
scheme is crucial for the accuracy of the filtration.

In this chapter, we also showed that the inner structure of a porous medium strongly influences the deposition of particles. In both in-line and staggered geometries filtration appears to depend strongly on particle inertia. A relatively mild dependence on St was observed for the in-line geometry. On the other hand, particle deposition in the staggered geometry showed strong dependence on the particle inertia. As a result, a critical particle Stokes number was observed, which separates two regimes. Particle with $S t \geq 0.04$ are filtered with high efficiency, while particles with $S t<0.04$ are filtered at considerably lower rate. This suggests that the staggered geometry can be used to separate particles with Stokes number below a critical value from larger particles. The critical Stokes number is observed at an overall porosity of $3 / 4$ - it is expected to depend on this porosity, flow conditions and inner structure of the porous medium, yielding several additional design parameters for particle filters.

Based on the IB method for predicting flow in porous media, it is possible to consider any complex porous medium, e.g., obtained using micro-computed tomography. Since the 'no-slip consistent' particle tracking is formulated entirely in terms of the phase-indicator function related to the geometry, our method is directly adaptable without any further alterations required. The method allows one to investigate the subtleties of filtration characteristics of a porous filter, depending on the flow conditions, porosity and particle size. Further research is devoted to the inclusion of Brownian motion into the particle dynamics, and to the application of this approach to filtration characteristics of realistic filters. 


\title{
Chapter 3
}

\section{Diffusive and inertial impaction filtration of aerosol droplets by porous media}

\begin{abstract}
In this chapter we address filtration of droplets whose dynamics is governed by both Stokes drag and Brownian motion. Within the Euler-Lagrangian framework of gasdroplet two-phase flow, the effects of inertial motion and Brownian diffusion on the filtration characteristics are first illustrated for flow through a straight pipe, serving also as validation of the model. Subsequently, the filtration characteristics of a steady flow through a realistic porous material are determined, illustrating the capabilities of the approach in terms of predicting such macroscopic aspects based on pore-resolved flow. We show a characteristic 'V-shaped' dependence of the filtration efficiency on the droplet size with high values in case of dominant Brownian motion or dominant inertial motion and a strongly reduced filtration efficiency for droplets of intermediate size.
\end{abstract}

\subsection{Introduction}

In various areas, such as chemical engineering, mechanical engineering and environmental sciences, prediction of the deposition rate of aerosol droplets on porous (fibrous) filters plays and important role. Detailed understanding of deposition characteristics on fibrous (porous) filters may allow more control over the filtration process. 
In this chapter, the transport and deposition of dispersed liquid droplets embedded in a gas flow through a porous material is studied using numerical simulations. We adopt an Euler-Lagrange formulation and incorporate explicitly Brownian forcing next to viscous Stokes drag. The filtration characteristics of a laminar flow through a realistic porous material are predicted through these simulations.

We present a numerical approach to study filtration characteristics of fibrous (porous) filters due to inertial impaction and diffusion. We consider one-way coupling of the gas phase and the particle phase, where the gas phase is governed by the Navier-Stokes equations [44], [13] and the dynamics of the particle phase is based on the Maxey-Riley equation [34]. The inner geometry of the filter is incorporated in our model using the immersed boundary (IB) [36] volume penalization technique [2], allowing to consider complex flow domains at full spatial resolution. The motion of small spherical particles in a fluid flow is approximated by retaining only the Stokes drag term given that the ratio of particle density to fluid density is large. Adopting this simplification and also including a random forcing to model Brownian motion, a stochastic equation for the motion of individual particles is obtained. These two forces represent the two main filtration mechanisms that will be investigated in this chapter, i.e., inertial impaction filtration associated with Stokes drag and diffusive filtration associated with thermal fluctuations representing Brownian forcing.

The collection efficiency of a filter may be influenced by several factors, such as packing density of the fibers, gas flow rate, temperature of the gas, particle size, etc., as these factors define the relative importance of the two dominant filtration mechanisms. The detailed understanding of the flow field in the filter and its impact on the particle motion is essential to calculate the collection efficiency of a given filter. The relative importance of the inertial and Brownian terms is directly connected to the particle diameter $d_{p}$ and the temperature $T$. We will quantify this momentarily, showing an increased Brownian effect with increasing temperature $T$ and/or decreasing particle size. Through systematic simulations the consequences of these mechanisms to the overall filtration will be quantified.

The underlying dependencies on the particle size imply the existence of several 
filtration regimes. While inertial forces are dominant for relatively large particles, Brownian forces dominate for small particles. In the absence of Brownian motion, small particles mainly follow the flow streamlines avoiding any obstacles. Trajectories of large particles, on the other hand, deviate from the flow streamlines and thereby may encounter obstacles. In the presence of Brownian acceleration, relatively small particles are affected by the thermal motion of gas molecules and their trajectories are no longer strictly following the flow streamlines. For the large size particles inertia remains the main reason for deviation from gas streamlines.

Intuitively, there should exist an intermediate range of particle sizes for which both the Stokes drag and the Brownian forces are of comparable importance. This suggests a V-shaped filtration efficiency curve as function of a particle size. While considerable filtration efficiency is expected for very small particles, arising from the Brownian motion, and for very large particles, arising from 'ballistic' motion through the fibrous medium, a reduced filtration efficiency is expected for intermediate range particles. The aim of the study presented here is to quantify the precise dependencies on particle size and system temperature. Confirmed by our simulations, both light (diffusion dominated) and heavy (inertia dominated) particles are captured with higher probability than intermediate sized particles.

The chapter is organized as follows: in Section 3.2 we briefly give the mathematical description of the gas-particle two-phase flow and discuss the numerical methods used to solve the corresponding system of equations and validate the approach on the basis of Brownian motion in one-dimension. Next, in Section 3.3 we illustrate the approach for Poiseuille flow in a tube. Section 3.4 is devoted to the application of the method to a real filter geometry for which we estimate the filtration efficiency as function of the particle size. Finally, we conclude with a summary of our findings in Section 3.5. 


\subsection{Modeling the motion of small aerosol droplets due to inertial and random forces}

In this section, we describe the mathematical model for simulating gas-particle twophase flow through porous media. We first present the governing equations for both phases and then turn our attention to the corresponding numerical methods, used to obtain the solution. Finally, we validate the approach using 1D Brownian motion and compare this to diffusion.

\subsubsection{Eulerian description of the gas-phase}

To model aerosol filtration by porous filters, we start with the mathematical modeling for the carrier phase. We assume a dilute multiphase flow for which the fluid velocity can be computed ignoring the droplet-phase [10]. The governing equations for the gas phase are the incompressible Navier-Stokes equations. We assume that the physical domain $\Omega$ contains $N$ 'obstacles', which form the solid part $\Omega_{s}$ of our computational domain. We employ an IB volume penalization method $[36],[2]$ which allows computing the flow in complex geometries using fast and efficient numerical methods on Cartesian grids. The non-dimensional Navier-Stokes equations [44], extended to the whole computational domain, in vector form are:

$$
\left\{\begin{aligned}
\frac{\partial \mathbf{u}}{\partial t}+\mathbf{u} \cdot \nabla \mathbf{u} & =-\nabla p+\frac{1}{R e} \nabla^{2} \mathbf{u}-\frac{1}{\epsilon} \Gamma_{s} \mathbf{u} \\
\nabla \cdot \mathbf{u} & =0
\end{aligned}\right.
$$

to be solved for the unknown gas velocity $\mathbf{u}$ and pressure $p$. The penalization parameter $\epsilon \ll 1$ and $\Gamma_{s}$ is the characteristic function of $\Omega_{s}$. It is used to penalize the flow entering the solid obstacles and approximates the no-slip boundary conditions at all solid-fluid interfaces:

$$
\Gamma_{s}(x)=\left\{\begin{array}{lll}
1 & \text { if } & x \in \Omega_{s} \\
0 & \text { if } & x \in \Omega_{f}
\end{array}\right.
$$


with $\Omega_{f}$ being the part of the computational domain that is occupied by the fluid. The Reynolds number Re quantifies the ratio of convective and viscous fluxes and is defined as:

$$
R e=\frac{L U}{\nu}
$$

where $\nu$ is the kinematic viscosity and $L$ and $U$ are the characteristic length and velocity scales, respectively, yielding a natural time-scale $L / U$. We will consider laminar flow through porous filters, corresponding to relatively low values of $R e$. This aspect will be specified in more detail below.

\subsubsection{Lagrangian description of the droplet-phase}

The deposition of particles on the surface of a filter can be caused by different filtration mechanisms including inertial impaction and diffusion [23]. In both cases, particles are captured because their trajectories deviate from fluid streamlines close to the surface of the solid. In the case of impaction this is due to the particle inertia while in the case of diffusion the underlying Brownian motion is the cause. Depending on the particle inertia and the gas temperature one of these mechanisms may be dominant.

Particle inertia, or the particle's responsiveness to changes in the flow, is characterized by the Stokes number $(S t)$ : the ratio of the particle response time to the flow time scale. In the absence of Brownian motion, low inertia particles essentially follow the fluid flow and therefore are transported as fluid elements. Somewhat larger particles can, e.g., be trapped by vortices in the flow before possibly colliding with the fluid-solid interface. Trajectories of particles with large inertia will not be affected much by vortex structures, and follow more or less ballistic paths, rather independent of any surrounding flow of the carrier gas.

For droplets for which Brownian diffusion becomes pronounced, deposition can be viewed as a mass transfer problem, for which various Euler-Euler engineering models were proposed. This approach is limited to cases where particle collection by the filter is only due to Brownian diffusion. For more general cases, where deposition 
mechanisms other than diffusion are also present, considering the droplet phase as a discrete phase has marked advantages. Here, we consider the so-called Lagrangian description of the droplet phase. The motion of individual droplets is modeled, based on the Maxey-Riley equations [34], which is extended to include diffusive motion of particles. This provides a framework for studying the combined effect of Brownian motion and inertial forces on the particle deposition. The trajectories of individual particles are obtained using the following system of non-dimensional equations:

$$
\left\{\begin{array}{l}
\frac{\mathrm{d} \mathbf{x}}{\mathrm{d} t}=\mathbf{v} \\
\frac{\mathrm{d} \mathbf{v}}{\mathrm{d} t}=\frac{(\mathbf{u}-\mathbf{v})}{S t}+\mathbf{A}(t)
\end{array}\right.
$$

where $\mathbf{x}=\{x, y, z\}$ and $\mathbf{v}=\left\{v_{x}, v_{y}, v_{z}\right\}$ are the particle position and velocity, respectively, and $\mathbf{A}(t)=\left\{A_{x}(t), A_{y}(t), A_{z}(t)\right\}$ is the random Brownian acceleration vector. The dimensionless Stokes number $S t$ is defined as:

$$
S t=\frac{U}{L} \frac{1}{\beta}
$$

where the relaxation time $\beta$ with the use of Stokes' law is given as:

$$
\beta=\frac{3 \pi \mu d_{p}}{C_{c} m}
$$

with particle diameter $d_{p}$, Cunningham correction factor $C_{c}$, dynamic viscosity of the gas $\mu$ and particle mass $m$ [7]. The Cunningham correction factor is essential when calculating the drag on small particles to account for non-continuum effects and is defined as $[8]$ :

$$
C_{c}=1+\frac{2 \lambda}{d_{p}}\left(1.257+0.4 \exp \left(\frac{-1.1 d_{p}}{2 \lambda}\right)\right)
$$

where $\lambda$ is the free mean path of the gas molecules. The gas-phase velocity $\mathbf{u}$ is evaluated at the particle location, i.e., $\mathbf{u}(\mathbf{x}(t), t)$. The relaxation time can be rewritten 
as follows:

$$
\beta=\frac{18 \mu}{C_{c} \rho_{p} d_{p}^{2}}
$$

where we introduce the particle density $\rho_{p}$ and substitute $m=d_{p}^{3} \pi \rho_{p} / 6$.

The random Brownian acceleration vector $\mathbf{A}(t)$ is assumed to be independent of $\mathbf{v}(t)$ and fluctuates rapidly as compared to the variations in $\mathbf{v}$. We consider a small time interval $\Delta t$, which on the one hand is short enough such that during this interval the external velocity $\mathbf{u}$ can be approximated as constant, while on the other hand it is long enough such that a large number of random accelerations took place. Under these conditions the formal solution of (3.2) for the interval $\Delta t$ is given by [5]:

$$
\mathbf{v}(\Delta t)=\mathbf{v}(0) \exp (-\Delta t / S t)+\mathbf{u}(1-\exp (-\Delta t / S t))+\mathbf{R}_{v}(\Delta t)
$$

where $\mathbf{R}_{v}(\Delta t)$ represents the net accumulated acceleration experienced by a Brownian particle during the time interval $\Delta t$ and $\mathbf{v}(0)$ is the particle's velocity vector at the beginning of the time interval. The random variable $\mathbf{R}_{v}$ has probability density function $w\left(\mathbf{R}_{v}\right)$ given by [5]:

$$
w\left(\mathbf{R}_{v}\right)=\frac{1}{\left(2 \pi\left(\frac{k_{B} T}{m U^{2}}\right)(1-\exp (-2 \Delta t / S t))\right)^{3 / 2}} \exp \left(\frac{-\left|\mathbf{R}_{v}\right|^{2}}{2\left(\frac{k_{B} T}{m U^{2}}\right)(1-\exp (-2 \Delta t / S t))}\right)
$$

This implies that each component of $\mathbf{R}_{v}=\left\{R_{v x}, R_{v y}, R_{v z}\right\}$ follows a normal distribution $N\left(0, \sigma^{2}\right)$ with zero mean and variance $\sigma^{2}$ :

$$
\sigma^{2}=\left(\frac{k_{B} T}{m U^{2}}\right)(1-\exp (-2 \Delta t / S t))
$$

which emphasizes the important parameter $\left(k_{B} T\right) /\left(m U^{2}\right)$, the ratio between the thermal energy scale $k_{B} T$ and the kinetic energy scale $m U^{2}$. The $x$ component of the solution vector given by (3.7) can then be written as follows:

$$
v_{x}(\Delta t)=v_{x}(0) \exp (-\Delta t / S t)+u_{x}(1-\exp (-\Delta t / S t))+\sigma N(0,1)
$$


where we employ the fact that the statistical properties of a random variable $X$ with probability distribution function $N\left(0, \sigma^{2}\right)$ are identical to the statistical properties of a random variable $Y$ with a probability distribution $\sigma N(0,1)$ [48].

\subsubsection{Numerical solution of governing equations}

We discuss the numerical technique for solving the formulated system of equations. We first turn to the numerical solution of the Navier-Stokes equations.

The IB technique to compute the gas flow around solid obstacles embedded in the domain allows to employ Cartesian meshes on which the governing equations are solved. For simulation of an incompressible flow a finite-volume discretization of the equations (3.1) is used [44]. We adopt a staggered storage of variables, which helps avoiding unphysical pressure fields that might result from the segregated pressure solving step [13]. In the 3D case, the scalar variables, such as pressure, are stored in the middle of the grid cells, while the velocity components are stored in the center of the corresponding faces of the grid cells.

Discretization of the Navier-Stokes system requires approximation of the differential operators appearing in the equations. The skew-symmetry of the differential operator $(\mathbf{u} \cdot \nabla)$ implies that the total energy of the flow is conserved when the flow is inviscid. It only decreases when there is dissipation. To achieve this on the discrete level a discrete skew-symmetric approximation of the operator $(\mathbf{u} \cdot \nabla)$ and a positivedefinite approximation of $-\nabla \cdot \nabla$ are developed in [56] which we employ in this work. The numerical method is identical to that used in [31, 20].

The numerical treatment of the equations of motion for the droplets will be discussed next, assuming steady flow for convenience. This assumption is not a principal limitation of the method and extension to time-dependent flow is readily made. Based on the exact solution given by Equation (3.10), a numerical scheme can be developed for computing the particle's position and velocity for sufficiently small time intervals $\Delta t$. Given the particle's position and velocity at time $t$, Equation (3.10) can be used to compute the particle's velocity at $t+\Delta t$. Below we write down the scheme for the $x$-component of the position and velocity vectors, where we adopt the Euler-forward 
method for evaluating the particle's position:

$$
\left\{\begin{aligned}
x^{n} & =x^{n-1}+\Delta t v_{x}^{n-1} \\
v_{x}^{n} & =v_{x}^{n-1} \exp (-\Delta t / S t)+u_{x}\left(\mathbf{x}^{n-1}\right)(1-\exp (-\Delta t / S t)) \\
& +\sqrt{\left(\frac{k_{B} T}{m U^{2}}\right)} \sqrt{1-\exp (-2 \Delta t / S t)} g^{n}
\end{aligned}\right.
$$

with $g^{n}$ a random number drawn from $N(0,1)$. The time step $\Delta t$ is assumed to be small compared to the particle relaxation time. This implies, that $\Delta t / S t \ll 1$. For this limit, by approximating $\exp (-\Delta t / S t)=1-\Delta t / S t$, we can rewrite (4.59) in the form of the Euler-forward scheme both for the position and the velocity as follows:

$$
\left\{\begin{array}{l}
x^{n}=x^{n-1}+\Delta t v_{x}^{n-1} \\
v_{x}^{n}=v_{x}^{n-1}(1-\Delta t / S t)+u_{x}\left(\mathbf{x}^{n-1}\right) \Delta t / S t+\sqrt{\left(\frac{2 \Delta t}{S t}\right)\left(\frac{k_{B} T}{m U^{2}}\right)} g^{n}
\end{array}\right.
$$

To assure stability of the explicit Euler-forward method for the velocity, the condition $\Delta t \leq 2 S t$ has to be satisfied which implies very small time steps for low values of the Stokes number. In order to propagate the particle position and velocity over a time step, the velocity of the gas at the particle position is required. For this purpose the gas flow velocity is interpolated to the current particle position. Here we use trilinear interpolation.

\subsubsection{Validation of Brownian motion in 1D}

Let us consider the motion of $N$ particles in 1D under a random Brownian force, assuming a gas velocity $u=0$. We simulate the motion of $N$ particles having the same initial position and velocity, given by $x_{j}(0)=0, v_{j}(0)=0, j=1, \cdots, N$ with diameter $d_{p}=1 \mu \mathrm{m}$. We assume a constant gas temperature $T=300 \mathrm{~K}$ and take a time step $\Delta t=10^{-6} \mathrm{~s}$. It is known that the root of the variance of the particle 


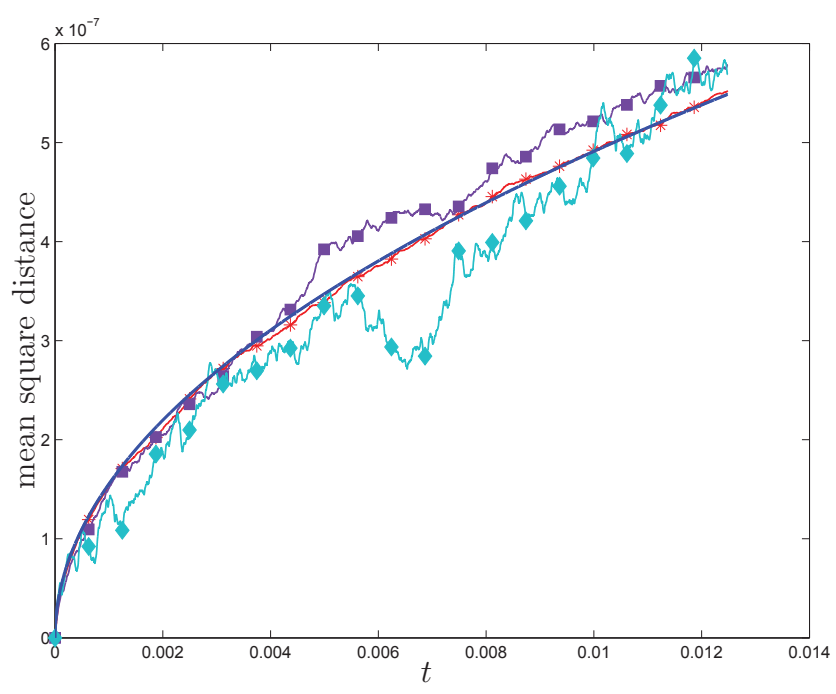

Figure 3-1: Numerically obtained root-mean-square displacement and theoretically predicted (solid) for $N=10^{3}$ (star), $N=10^{2}$ (square), $N=10$ (diamond) particles with diameter $d_{p}=1 \mu \mathrm{m}$.

position $\lambda$ on average grows as the square root of time [5]:

$$
\lambda(t)=\sqrt{2 D} \sqrt{t}
$$

where $D$ is the diffusivity coefficient, which is given by Stokes-Einstein expression:

$$
D=\frac{k_{B} T}{6 \pi d_{p} \mu}
$$

with $\eta$ the viscosity of the gas. We consider $T=300 \mathrm{~K}$, take the viscosity of air $\mu=1.98 \times 10^{-5} \mathrm{~kg} /(\mathrm{m} \cdot \mathrm{s})$ and mass density $\rho_{p}=1000 \mathrm{~kg} / \mathrm{m}^{3}$. The numerically predicted root-mean-square of the displacement is defined as:

$$
\lambda_{\text {num }}^{n}=\frac{\sqrt{\sum_{j=1}^{N}\left(x_{j}^{n}-x_{j}(0)\right)^{2}}}{\sqrt{N}}
$$

In Figure 3.2.4 we compare the numerically obtained mean square displacement with the theoretical prediction. With $N$ increasing the numerical curve for the rootmean-square displacement converges to its theoretical prediction. We have also established that by taking a sufficiently small time step, taken as a fraction of $S t$, the two 


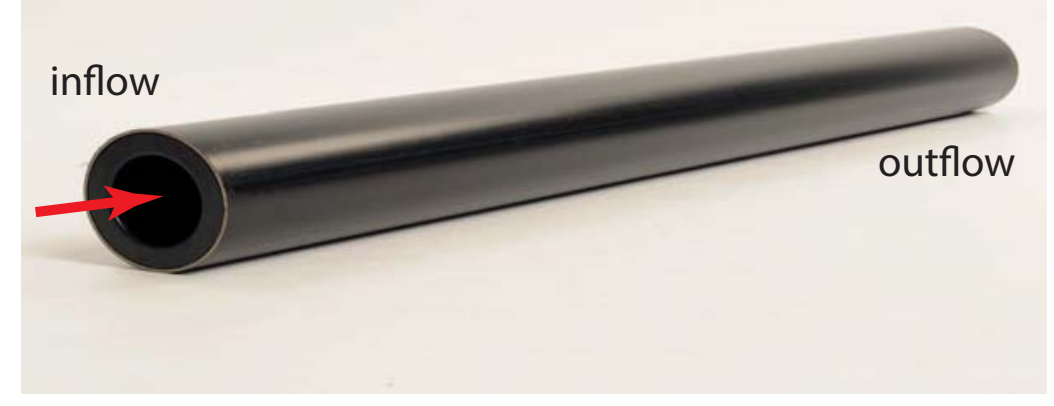

Figure 3-2: Illustration of the flow domain with particles entering with the flow at the inflow boundary and leaving via the outflow boundary. At the inflow a Poiseuille profile is imposed while at the outflow the derivative of the solution with respect to the streamwise coordinate is set to zero.

numerical solutions based on the full Euler-forward scheme given by (3.12) and on the exact solution computed by (3.11) converge likewise. Having a proper description of the motion of Brownian particles, we next turn our attention to the application of this method.

\subsection{Filtration in a circular tube}

The objective of this section is to illustrate the developed numerical model on the example of flow in a circular tube. In this relatively simple geometry, we aim to qualify filtration characteristics due to diffusion and inertial impaction and understand the effects of the underlying physical mechanisms. This configuration is well suited for validation purposes, a necessary step toward the application of this method to filtration by realistic fibrous filters discussed in the next section.

Before going into the details of the setup for the numerical experiments, we introduce some notation. Initially, a single 'cloud' of $N_{\text {in }}$ particles is introduced near the inlet plane of the computational domain (see the sketch of the computational domain in Figure 3-2). This number of particles is followed in time - no further particles are added at later times. We are interested in the number $N_{c a p}(t)$ of particles deposited 
on the wall of the tube. In addition, we denote with $N_{e s c}(t)$ the number of particles that remain non-captured when exiting the tube. At any given time $t$ :

$$
N_{\text {in }}=N_{\text {cap }}(t)+N_{\text {esc }}(t)
$$

Based on these notations, we define the penetration of particles $P$ as follows:

$$
P(t)=1-\frac{N_{c a p}(t)}{N_{i n}}
$$

According to this definition, $P=1$ implies that the number of deposited particles up to time $t$ is zero. Correspondingly, $P=0$ implies that all of the particles have been deposited at time $t$.

The circular tube has an inner diameter and length of $l_{t}=7.8 \mathrm{~mm}$ on which we base the length scale $L=l_{t}$. For the velocity reference scale we assume $U=0.28 \mathrm{~m} / \mathrm{s}$ while the particle density is taken as $\rho_{p}=1000 \mathrm{~kg} / \mathrm{m}^{3}$ and air viscosity as $\nu=15 \cdot 10^{-6}$ $\mathrm{m}^{2} / \mathrm{s}$, which is valid for system temperatures $T \approx 300 \mathrm{~K}$. The Reynolds number $R e$ of the flow, based on the chosen length and velocity scales, is approximately 145. A pressure difference is maintained over the length of the domain in the $x$ direction to force the flow. At the inflow we impose a Poiseuille flow profile for the velocity, while at the outflow the derivative with respect to the streamwise coordinate is set to zero.

The numerical prediction for the laminar velocity profile in Poiseuille flow is illustrated in Figure 3-3. Note that the flow is computed numerically for a resolution that provides a fair approximation to the analytical solution [35]. Particles are introduced in the fluid domain at random locations, such that particles cover the fluid domain statistically uniformly and no clustering of particles arises. The initial velocity of a particle is taken equal to the local velocity of the fluid. By tracking the particle trajectories, we can determine where and when particles collide with the tube wall.

First, we look at the snapshots of the locations of $N_{i n}=10^{4}$ particles with particle diameter $d_{p}=0.1 \mu \mathrm{m}$ that are initially randomly distributed in the $y-z$ plane at $\{x=0.125\}$ (Figure 3-4). Simulations are performed until $t=2$ non-dimensional time units, which corresponds approximately to $1 / 2$ a flow-through time, i.e., half 


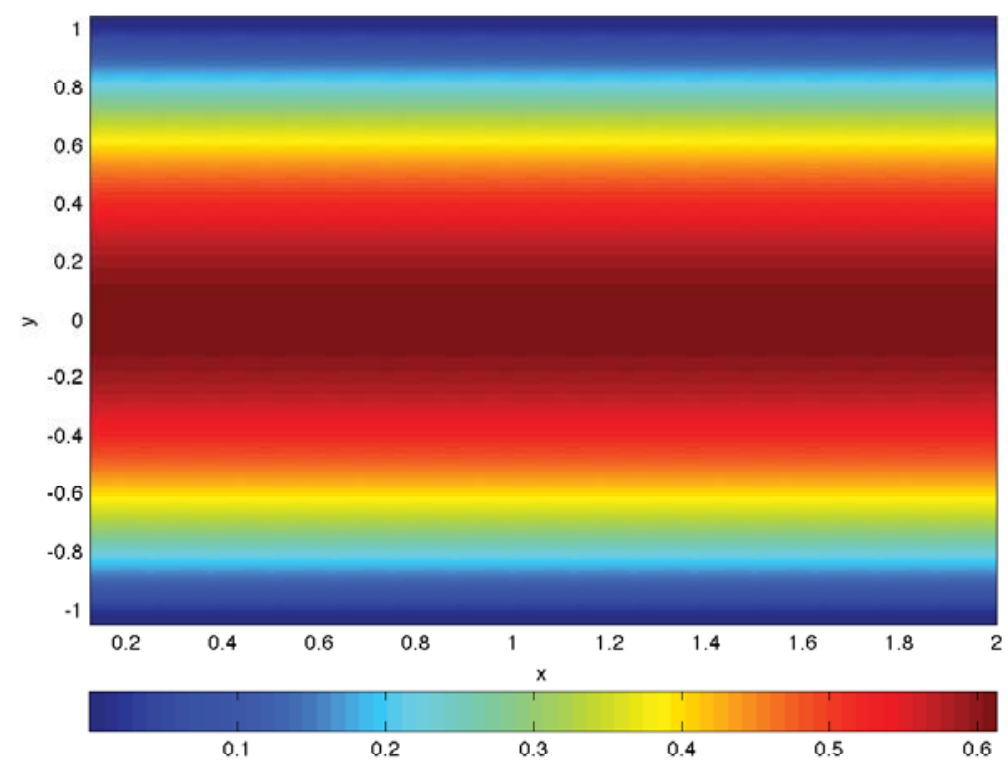

Figure 3-3: Flow field in the circular tube. Velocity components in the $x$ and $y$ directions are presented for $R e=145$ at resolution of $32 \times 64 \times 64$.

the time spent by a particle to travel through the domain in the streamwise direction in case it would travel with the reference velocity $U$. For this particle diameter and reference temperature, it appears that Brownian acceleration is not strong and the trajectories are not affected much as the particles migrate towards the outlet of the channel following the parabolic profile of the gas velocity field.

In this simple geometry, deposition arises mainly due to diffusion, which is dominant for the motion of relatively small particles. Without diffusion no particles would encounter the walls as, initially, no radial velocity component was given to the particles. The only mechanism that makes the particles deviate from a purely streamwise motion is Brownian acceleration. This is confirmed by evaluating the dependency of the penetration on the particle size for fixed gas temperature of $300 K$ (see Figure $3-5)$. For the smallest particle sizes considered here, $d_{p}=0.06 \mu m$, only $0.3 \%$ of the particles are captured. We establish that for particles with $d_{p} \geq 0.11 \mu \mathrm{m}$ less than $0.1 \%$ of the particles gets captured. These very low values of the captured fraction is due to the rather modest temperature, but also due to the short domain length that was chosen in this illustration. In Figure 3-5 we notice that the penetration $P$ depends monotonously on the particle size, i.e., with decreasing size the fraction of 


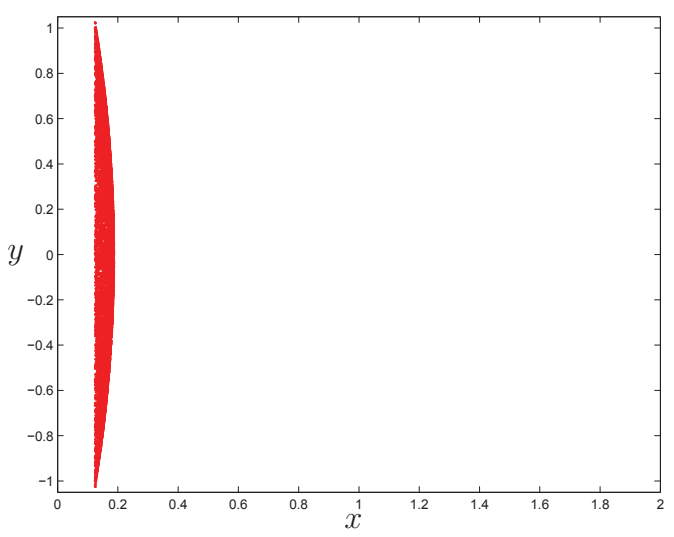

(a) $t=0.1$

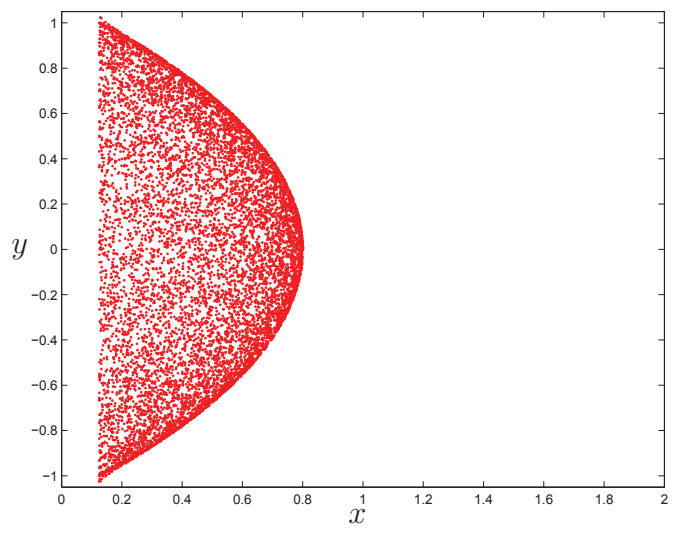

(c) $t=1$

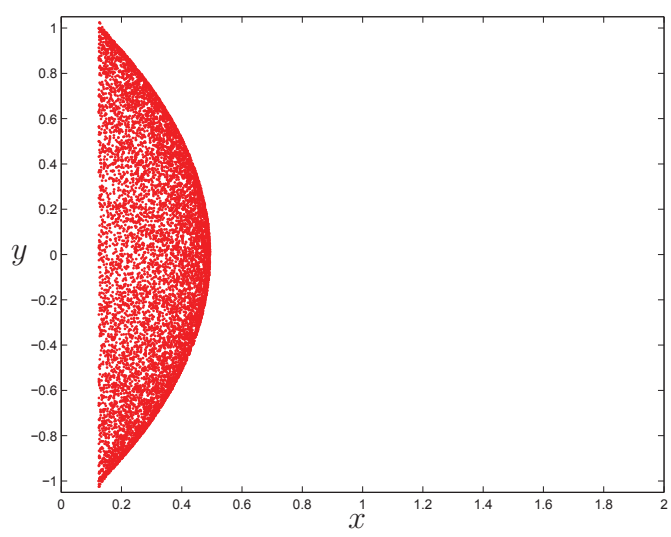

(b) $t=0.5$

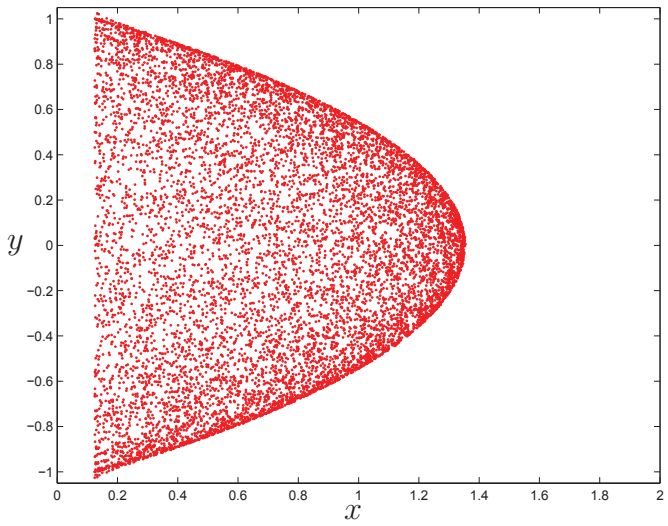

(d) $t=2$

Figure 3-4: Particle positions for particles with diameter $d_{p}=0.1 \mu \mathrm{m}$ in the circular tube. Initially, $10^{4}$ particles are randomly distributed in the $y-z$ plane at $x=0.125$. Initial velocity of the individual particles is taken equal to the linearly interpolated fluid velocity at the particle position. The locations of non-deposited particles (projected onto the $x-y$ plane) are registered at times $t=0.1, t=0.5, t=1$ and $t=2$. Only the particles that moved to the right are shown. 


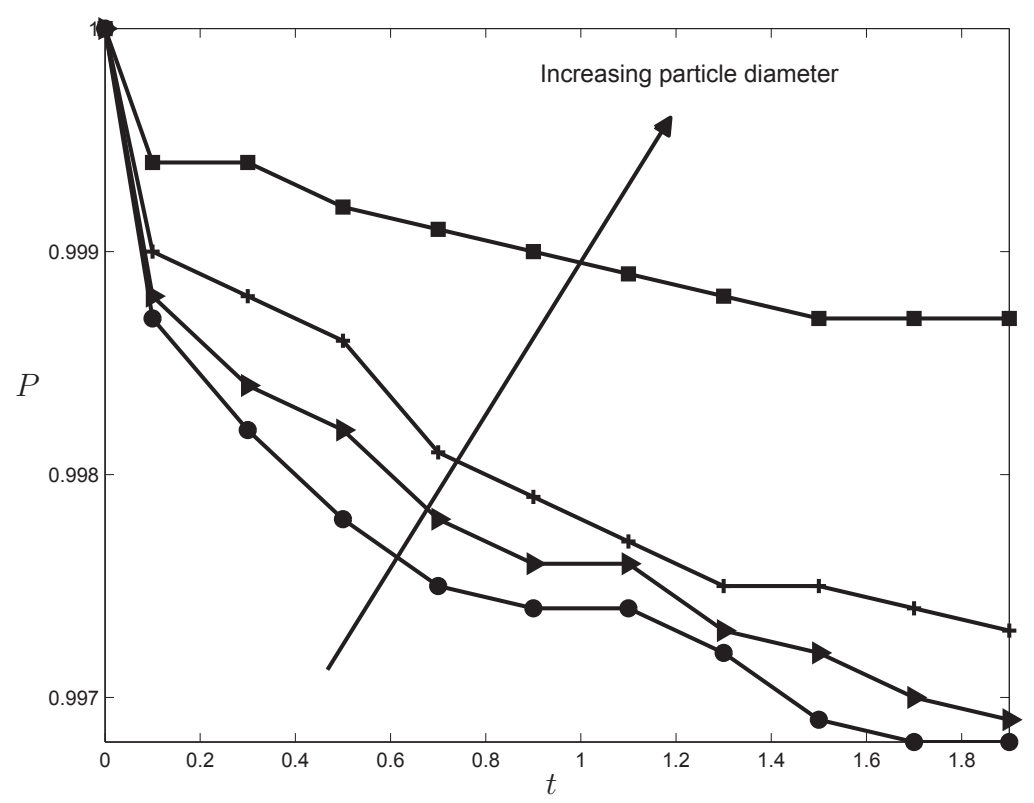

Figure 3-5: Penetration of particles as a function of time for different particle diameters for $T=300 \mathrm{~K}: d_{p}=0.06 \mu \mathrm{m}$ (circle), $d_{p}=0.07 \mu \mathrm{m}$ (triangle), $d_{p}=0.1 \mu \mathrm{m}$ (plus) and $d_{p}=0.2 \mu \mathrm{m}$ (square). Initial number of particles is $N_{i n}=10^{4}$.

captured particles $N_{c a p} / N_{\text {in }}$ increases, which is due to the increased relevance of the Brownian acceleration in view of the sharp increase in the parameter $k_{B} T /\left(m U^{2}\right)$ since $m \sim d_{p}^{3}$.

In Figure 3-6 we compare the penetration curves for gas temperatures $T=300 \mathrm{~K}$ and $T=600 \mathrm{~K}$ at particle diameter $d_{p}=0.06 \mu \mathrm{m}$. By increasing the gas temperature an increase in the deposition is observed. In fact, at $t=2$ we notice that the captured fraction $N_{c a p} / N_{\text {in }}$ has increased by roughly a factor 1.5 , which is comparable to $\sqrt{600 / 300}=\sqrt{2}$ governing the relative increase in the strength of the Brownian acceleration through the parameter $\sqrt{k T /\left(m U^{2}\right)}$ as is evident in (3.11) and (3.12).

In the following section, we consider a realistic filter geometry which implies richer particle dynamics and filtration trends. 


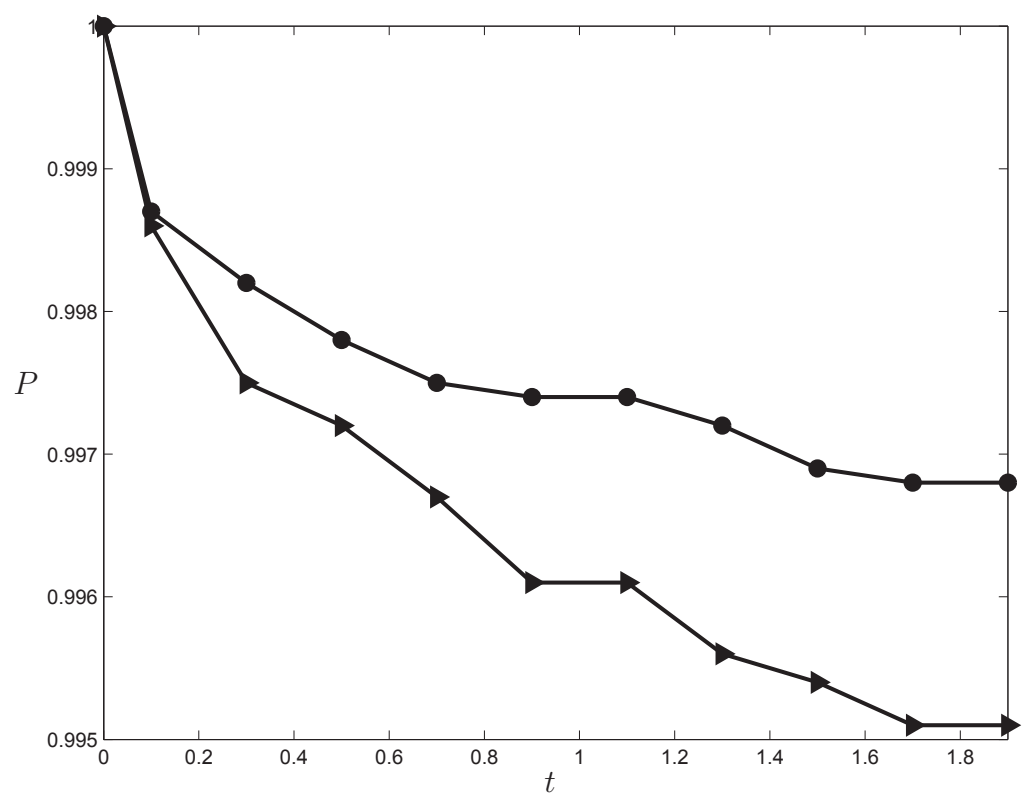

Figure 3-6: Penetration of particles as a function of time at different gas temperatures $T=300 K$ (circle) and $T=600 K$ (triangle) for particle diameter $d_{p}=0.06 \mu \mathrm{m}$. Initial number of particles is $N_{i n}=10^{4}$.

\subsection{Filtration in a porous filter}

In this section, we consider a realistic filter geometry and apply the developed numerical method to evaluate the particle removal efficiency. First, we describe the construction of the computational domain based on a realistic porous filter and then turn to numerical experiments performed to investigate its filtration efficiency.

\subsubsection{Construction of the computational domain}

The porous medium considered in this section is reconstructed from pore-scale geometric data obtained from micro-computed tomography: a set of 1536 images each having $664 \times 664$ pixels with a uniform width of $p_{w}=1.185 \cdot 10^{-2} \mathrm{~mm}$ are processed to construct a three-dimensional tube (using cubic voxels of volume $p_{w}^{3}$ ). The porous filter is essentially a tube filled with fibrous material forming the porous structure. In Figure 3-7 we show a representative cross sectional plot of the tube. The tube has an inner diameter $d \approx 7.8 \mathrm{~mm}$ and length $D=18 \mathrm{~mm}$ in the stream wise $x$-direction. On both sides of the tube we extend the computational domain by adding two empty 


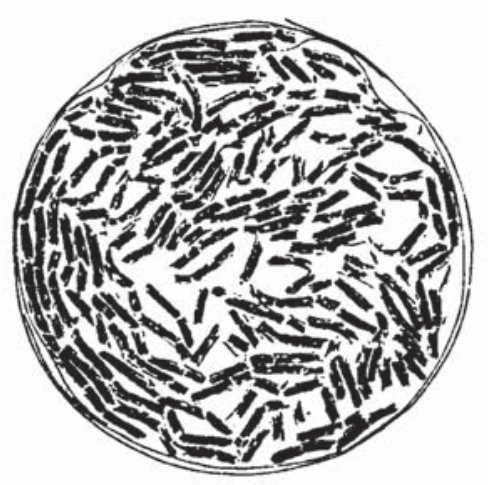

Figure 3-7: A representative cross-section of the tube with inner diameter of $7.8 \mathrm{~mm}$.

tube stretches of length $D / 6$ which serve as inlet and outlet regions [30]. Including the extensions, the total length of the tube becomes approximately $24.3 \mathrm{~mm}$.

In this chapter, results are obtained on a grid $n x=512$ and $n y=n z=128$. A detailed resolution study for the considered geometry and flow conditions was conducted showing that this resolution is high enough to capture the dominant transport characteristics [30]. We use this resolution to investigate the filtration characteristics of the porous filter.

The lower streamwise resolution is motivated by the fact that the fibrous porous material considered here is predominantly aligned with this axis. This representation was obtained by projecting the original $2048 \times(664)^{2}$ voxels onto this coarser resolution, which allows for systematic investigation of filtration properties for a broad range of particle sizes at acceptable computing times. At this spatial resolution, the fluid flow can be computed reliably, as was established in [30]. Based on the reference length scale taken as the diameter of the tube $L=d=7.8 \mathrm{~mm}=7.8 \cdot 10^{-3} \mathrm{~m}$ and velocity scale of $U=0.28 \mathrm{~m}$ (assuming a constant volumetric flow rate of $Q=1.75 \times 10^{4}$ $\mathrm{mm}^{3} / \mathrm{s}$ ), we compute the fluid flow through the porous tube at $R e=145$.

In Figure 3-8 and Figure 3-9 we present a visualisation of the computed velocity and the highly complex inner geometry of the porous domain. This result was obtained in [30] and serves as point of departure for our further analysis of the filtration efficiency of such a filter. We observe in the cross-section (Figure 3-8) that 


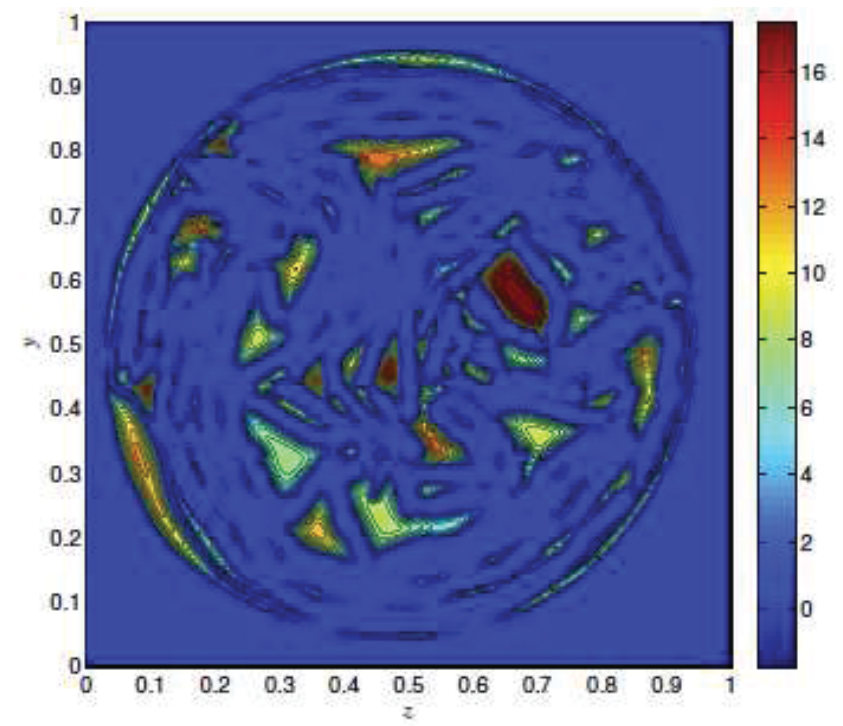

Figure 3-8: Contours of the dimensionless streamwise velocity-component in a representative cross-section of the tube. Image courtesy of [30].

the streamwise velocity is rather low in most of the domain, apart from small regions corresponding to the larger open pores contained in the structure. Hence, most of the transport of the gas through the porous domain occupies only a relatively small part of the entire volume. This is also observed in the streamwise cut through the domain in Figure 3-9 in which the slenderness of the pore structure is readily appreciated. Also in this cross-section one observes regions of relatively high velocity concentrated in the larger pores through the domain. The numerical accuracy of this solution was scrutinized in [30] in which it was shown that the immersed boundary method converges as a first order method for the velocity, yielding accurate findings for the permeability predictions agreeing better than $5 \%$ with experimental data obtained on exactly the same porous structure.

Next, we detail the numerical experiment that uses this velocity field to investigate filtration properties of the porous tube.

\subsubsection{Numerical prediction of the filtration efficiency}

First, we describe the numerical evaluation of the filtration efficiency, after which we visualise the dispersion of the droplets in the domain, followed by a quantitative 

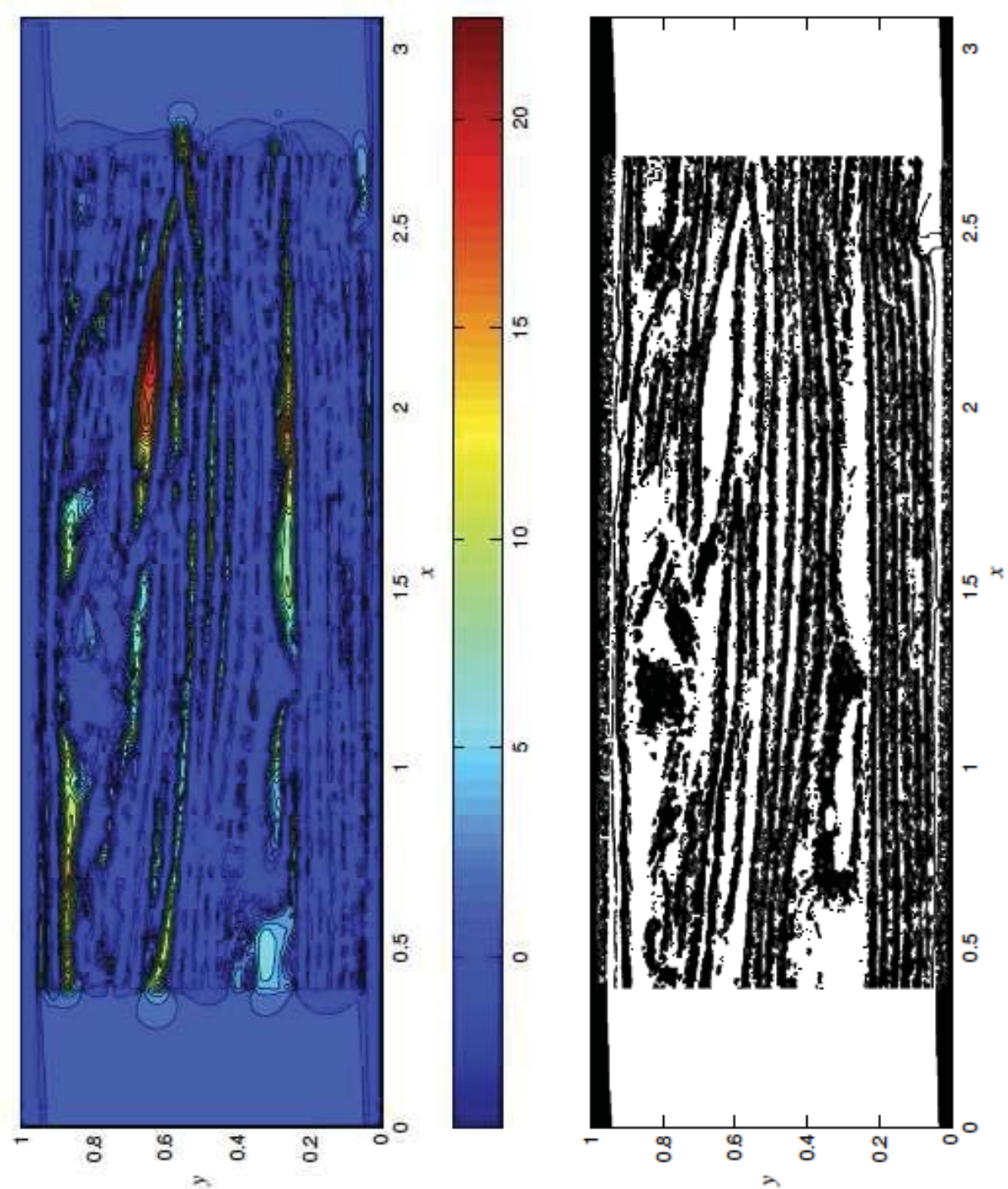

(a) Contours of the dimensionless streamwise (b) Black(solid) and white(void) repvelocity-component. resentation of the porous tube.

Figure 3-9: Representative cross-section in the streamwise direction. Image courtesy of $[30]$. 
analysis of the filtration.

The computational setup used for computing the filtration efficiency of the porous filter is similar to that used for the circular tube. At the inlet of the porous tube, approximately $D / 6$ into the computational domain and ahead of the actual fibrous plug, $N_{\text {in }}=10^{4}$ particles are introduced into the domain, initially randomly located in the cross-sectional plane defined by $x=0.125$. Unless mentioned otherwise, the simulation time corresponds to approximately $2 / 3$ of the flow-through time.

In Figure 3-10 we present snapshots of the locations of the non-captured and the captured particles. In this illustration, the particles have a diameter of $d_{p}=0.1 \mu \mathrm{m}$. As can be observed from these snapshots, there are apparent 'channels' through which a large fraction of the particles manages to propagate without being hindered much by the porous domain and even make it to the outflow boundary without getting captured. We observe that the captured particles accumulate for short times onto the leading face of the porous domain. At later times particles enter the pores and the deposited particles trace-out the complicated boundaries of the larger pores that make up the solid-fluid interface. Likewise, the non-captured particles move through the centers of these pores. Intuitively, this corresponds with the earlier observations of relatively high velocities in a small number of larger pores that pass through the full length of the domain.

To quantify the particle propagation through the porous tube, we performed simulations for a range of particle sizes $0.06 \mu m \leq d_{p} \leq 3 \mu m$. For selected particle sizes in Figure 3-11 penetration curves are plotted as a function of time computed for $\Delta t=10^{-7}$. Before we discuss the penetration curves, we briefly turn to the computational scheme used to compute the motion of particles. Comparing the computational schemes given by (3.11) and (3.12) at $\Delta t=10^{-7}$ showed similar penetration trends, however there is a slight quantitative difference in the actual penetration levels. In order to get better agreement between the two numerical schemes a smaller $\Delta t$ should be considered, i.e., implying a smaller value of $\Delta t / S t$.

Related to the initial positions of the particles it takes approximately until $t=0.1$ before the first particles reach the porous plug and get captured. This explains the 

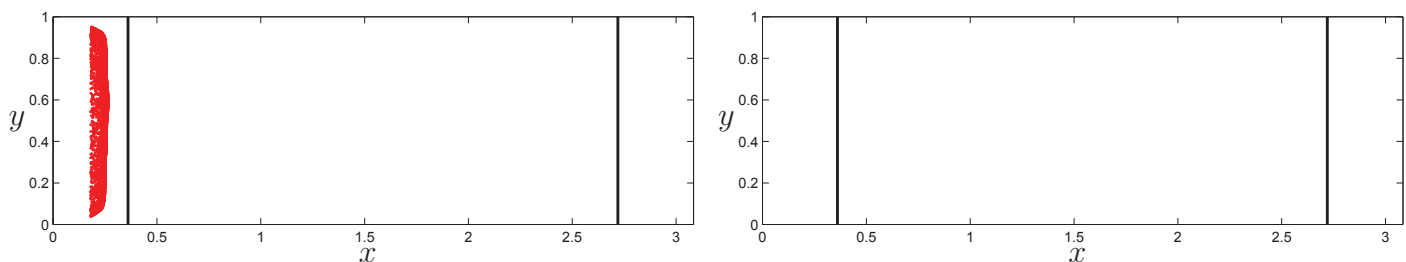

(a) $t=0.04$
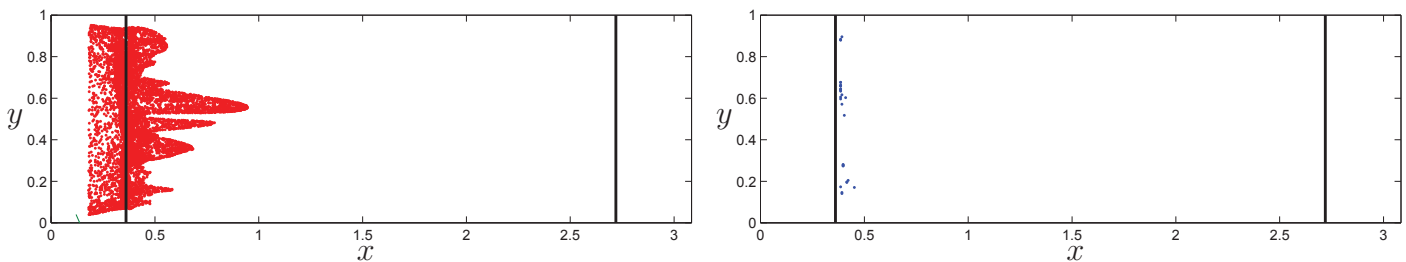

(b) $t=0.12$
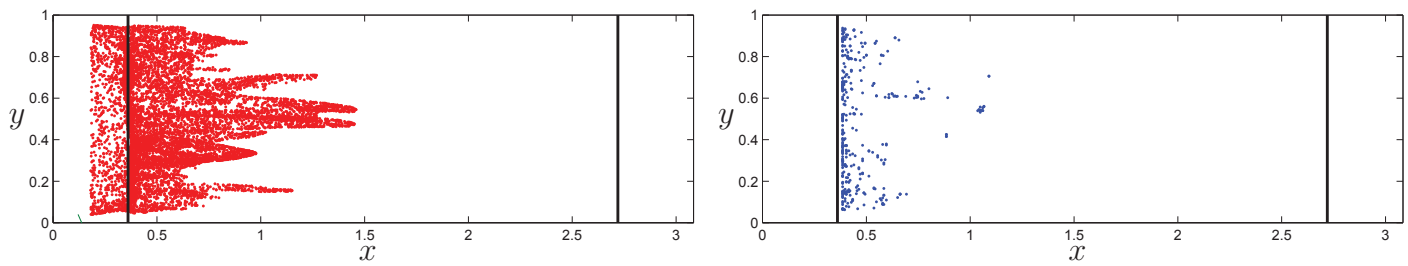

(c) $t=0.16$
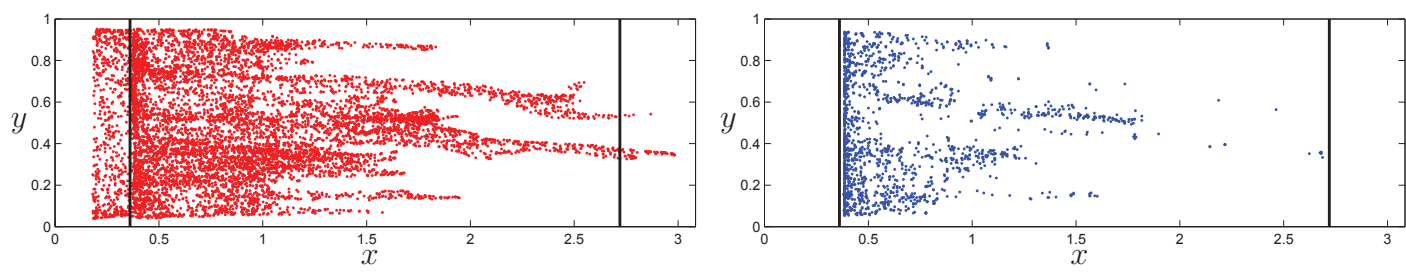

(d) $t=0.239$
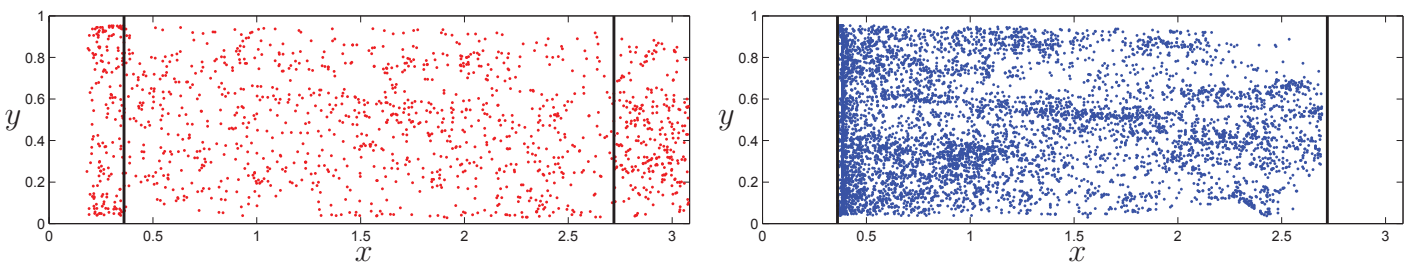

(e) $t=0.8$

Figure 3-10: Particle dynamics for $d_{p}=0.1 \mu \mathrm{m}$ in the realistic filter geometry. Initially, $10^{4}$ particles are randomly distributed in $y-z$ plane at $x \approx 0.125$. Initial velocity of the individual particles is taken equal to the interpolated fluid velocity at the particle position. The locations of non-deposited (red - left figures) and deposited (blue - right figures) particles are registered at times $t=0.04, t=0.12, t=0.16$, $t=0.24$ and $t=0.8$ (particle locations are projected onto $x-y$ plane). The solid lines indicate the start and the end of the porous part of the computational domain. 


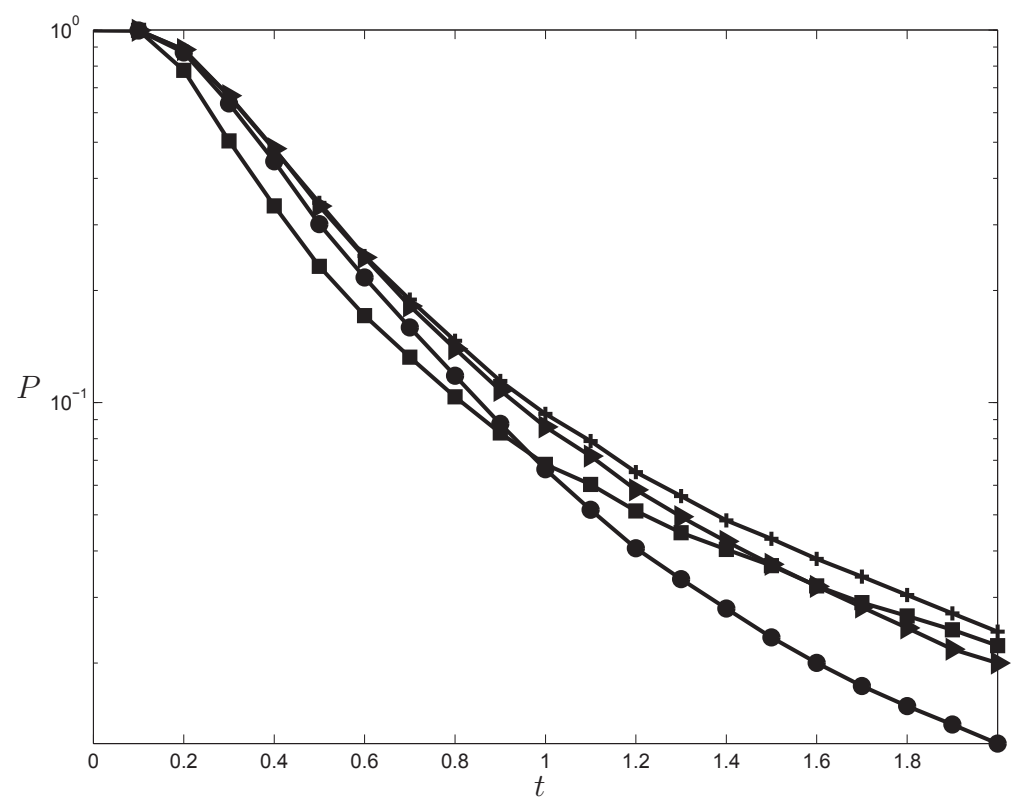

Figure 3-11: Penetration of particles as a function of time for different particle diameters at $T=300 \mathrm{~K}$ and $N_{i n}=10^{4}: d_{p}=0.06 \mu \mathrm{m}$ (circle), $d_{p}=0.1 \mu \mathrm{m}$ (triangle), $d_{p}=0.6 \mu m$ (plus) and $d_{p}=3 \mu m$ (square) .

flat initial part of the penetration curves. Compared to the validation case of a circular tube, we observe that the capturing of particles is much more efficient with the current fibrous porous material, compared to the empty tube case. While in the empty and shorter tube case only a fraction of a percent could be captured, we observe in this case a rapid increase of the number of captured particles to well above $90 \%$ of the initial number of particles that was put in the system. The penetration curve corresponding to a particle size of $0.1 \mu \mathrm{m}$ is slightly above the rest of the curves, indicating that particles with this size are captured less effectively compared to the rest. Marked with a plus symbol is the penetration curve computed for a particle size of $0.06 \mu \mathrm{m}$, the smallest particle size included in the study. Particles with this size are captured most effectively, due to the considerable influence of diffusion on the particle trajectories. Likewise, particles larger than $0.1 \mu \mathrm{m}$ are captured more accurately, reflecting the importance of inertia, which favours the particles to follow straight lines, quite independent of the embedding flow. In contrast to the case of the circular tube, here we observe a non-monotonous dependence of the filtration 
efficiency on particle size.

In the case of the circular tube, with decreasing particle size filtration becomes more effective uniformly. This is explained by the increased effect of Brownian motion, which takes particles away from the straight paths that would be followed under the action of Stokes drag alone. In the case of flow through a porous tube the motion of particles is influenced by the curved streamlines, corresponding to flow around the fibers. This results into a much richer particle dynamics implying different filtration regimes determined by the relative importance of forces acting on them.

The concept of removal coefficient can be used to describe the different filtration regimes, i.e., diffusion dominated, inertia dominated and 'mixed' in which case both contributions to the accelerations are dynamically relevant. In literature, filtration efficiency is often associated with the so-called removal coefficient $\gamma$. If we assume that the population of non-captured particles follows from a 'multiplicative process', and initially the distribution of particles is uniform over the domain, then an exponential decay would result. This assumes a homogeneous and isotropic porous medium with equal capturing probability throughout and connects mainly to the idea of random diffusive motion. If this would be a good approximation, then $\gamma$ expresses the speed of the decay, i.e.:

$$
P(t)=\exp (-\gamma t)
$$

The exponential decay model is only approximate and motivated for homogeneous porous media. As we deal with a porous plug with complex non-uniformities in the porosity, only a rough agreement between the simulated $P(t)$ and (3.17) was observed. Moreover, the level of agreement was dependent on the Stokes number of the droplets. This was confirmed at a range of droplet sizes. The exponential fit to the filtration curves did not allow a consistent level of approximation of the decay rate $\gamma$. However, the simulated filtration curves do give an indication of the efficiency of the filtration process and its dependency on the droplet size can be extracted, which can be used to optimize the design of a filter.

Relatively small particles are affected by Brownian acceleration and their trajec- 
tories are no longer following the flow streamlines. For large size particles their inertia is the main reason for deviation from streamlines. Moreover, an intermediate range of droplet sizes is observed for which a more close agreement between trajectories and streamlines arises. This yields a roughly V-shaped filtration characteristic (Cf. $[22,43,46])$ as a function of particle diameter, i.e,. a range of droplets is filtrated much less efficiently compared to very small (diffusion dominated) or rather large droplets (inertia dominated). We notice that an increase in the temperature from 300 $K$ to $600 K$ leads to a shift to higher filtration efficiencies for the smaller droplets, corresponding to the stronger contribution of the Brownian forcing. This establishes the principal suitability of the Euler-Lagrange method to understand porous filtration characteristics in detail - further development of the method and the simulation speed will be needed to make studies of realistic filtration feasible.

The V-shaped filtration efficiency is a result of the interplay of Stokes drag and Brownian forcing. In order to characterise the relative importance of these contributions to the velocities we extract their probability distributions from the simulations. We concentrate on the streamwise dynamics. The contributions to the velocity at time $t_{n}$ from Stokes' and Brownian forces (see equation 3.12) are denoted by $S^{n}$ and $B^{n}$, respectively:

$$
\begin{gathered}
S^{n}=v_{x}^{n-1}(1-\Delta t / S t)+u_{x}\left(\mathbf{x}^{n-1}\right) \Delta t / S t \\
B^{n}=\sqrt{\left(\frac{2 \Delta t}{S t}\right)} \sqrt{\left(\frac{k_{B} T}{m U^{2}}\right)} g^{n}
\end{gathered}
$$

To check the effect of gas temperature and particle size on the accelerations we compare findings for two gas temperatures,i.e., $T=300 \mathrm{~K}$ and $T=600 \mathrm{~K}$, and two particle sizes, i.e., $d_{p}=0.06 \mu \mathrm{m}$ and $d_{p}=0.1 \mu \mathrm{m}$. In Figures 3-12 particles' velocity distributions in terms of $B^{n}$ and $S^{n}$ are shown for all considered cases. As it can be observed, for all considered particle sizes the Brownian term remains smaller than the Stokes term. By increasing the gas temperature from $300 \mathrm{~K}$ to $600 \mathrm{~K}$ we essentially increase the Brownian term by a factor of $\sqrt{2}$. This, however, does not effectively change the relative values of the two velocity terms. The increase in temperature increases the span of the Brownian velocity term, however for the 

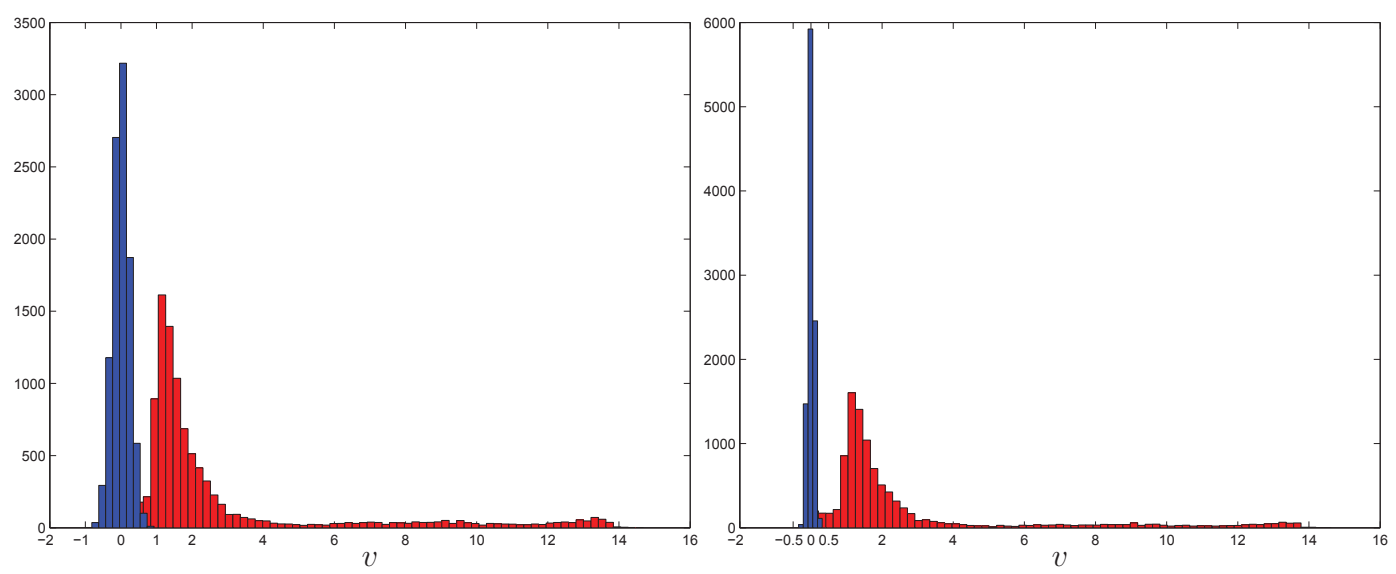

(a) $T=300 \mathrm{~K}$
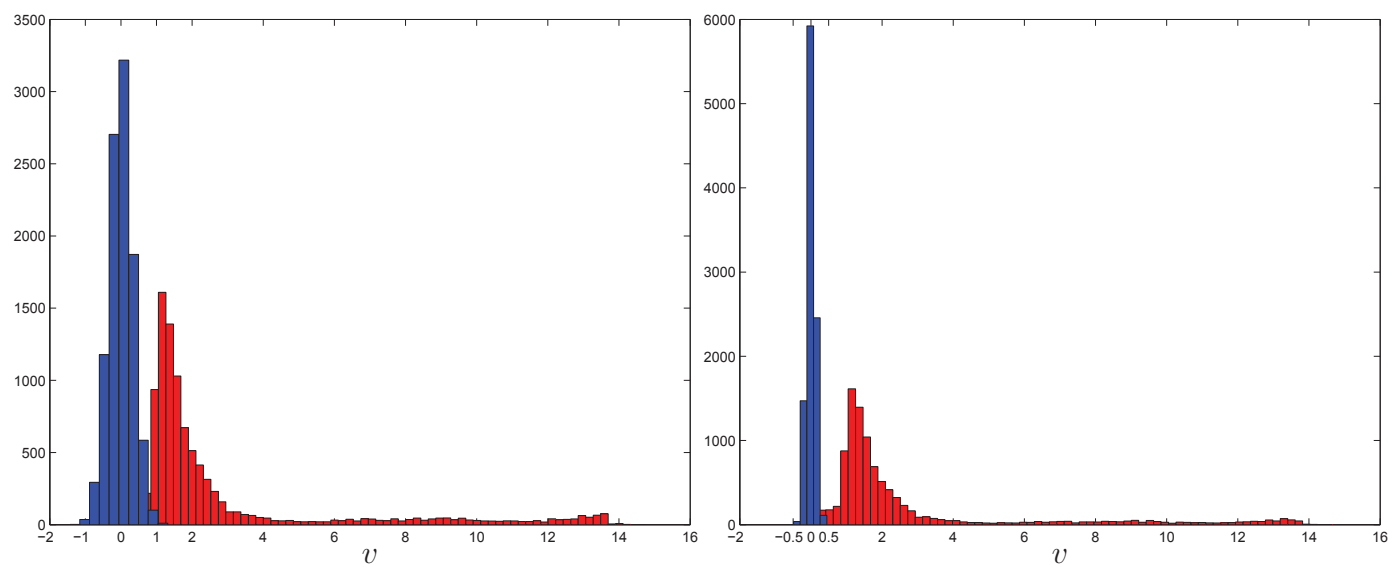

(b) $T=600 K$

Figure 3-12: Particle velocity distribution for particle diameter $d_{p}=0.06 \mu m$ (left figures) and $d_{p}=0.1 \mu \mathrm{m}$ (right figures) for two different gas temperatures indicated beneath the plots. Stokes velocity is shown in red and Brownian velocity term in blue. 


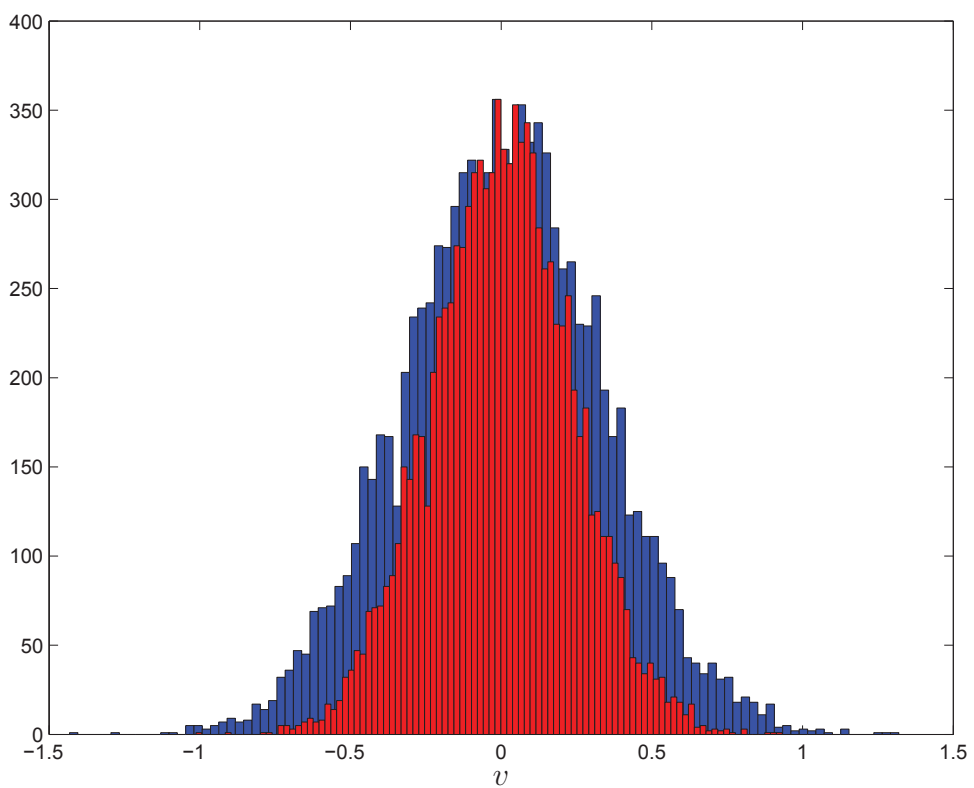

Figure 3-13: Brownian term $\left(B^{n}\right)$ of particle velocity ( $x$-component) for particle diameter $d_{p}=0.06 \mu \mathrm{m}$ for different gas temperatures: $T=300 \mathrm{~K}$ (red) and $T=600$ $K$ (blue).

majority of particles this term remains small relative to the Stokes term for both particles with $d_{p}=0.06 \mu \mathrm{m}$ and $d_{p}=0.1 \mu \mathrm{m}$. This can be better appreciated in Figure 3-13 where we compare the distributions of Brownian term with increasing temperature for $d_{p}=0.06 \mu \mathrm{m}$. Only a substantial increase of the gas temperature may result in more significant changes in the particle dynamics, even to the point that the Brownian terms become dominant. However, this would require a physically infeasible temperature range, to which we do not pay further attention.

\subsection{Conclusions}

The capability of predicting the complete flow, down to the pore scale, that arises in realistic fibrous porous media was exploited to study the filtration characteristics of such a filter. At different temperatures and particle sizes the capturing of droplets onto the solid-fluid interface was simulated and the filtration efficiency recovered. A classical 'V-shaped' filtration curve was observed as function of droplet size with high likelihood of filtration both for sufficiently small (dominant diffusion) as well as 
for sufficiently large (dominant inertial effects) droplets. The droplet sizes for which filtration is least effective were found to be in the sub-micron range, consistent with the typical ranges found in other filters. This builds some confidence in the accuracy of the model and motivates its use in the study and optimisation of mechanical filters. Particularly, the recovery of information such as where in the domain the dominant filtration takes place, is essential in case filtration characteristics need to be improved for certain size classes. However, further research is needed to better understand the dependence on parameters and to reach higher statistical reliability through the use of more particles and more repetitions of the numerical experiments to allow proper averaging. 


\title{
Chapter 4
}

\section{Numerical simulation of aerosol}

\section{formation and growth in laminar}

\section{flow}

\begin{abstract}
This chapter deals with an Euler-Lagrange model of the full set of processes of aerosol nucleation, growth by condensation and evaporation, and motion governed by Stokes drag as well as Brownian forces. This model is applied to laminar flow through a channel. The flow contains dibutylphthalate (DBT) vapor at the inflow, which is rapidly cooled at some location along the channel, thereby inducing droplet nucleation. While work presented in literature mainly deals with an Euler-Euler formulation, in this chapter we show results based on the Euler-Lagrange formulation in which the trajectories of nucleated droplets are followed precisely in time. We may predict the size distribution and other statistical properties of the aerosol inside the channel and also at its exit, starting from a dispersed particle description.
\end{abstract}

\subsection{Introduction}

Nucleation plays an important role in atmospheric processes [45, 24] and it is a crucial concept in the production of various polymer, alloy and ceramic materials [1, 25]. Nucleation is a key mechanism of phase transition in materials, e.g., it relates to the process of formation of liquid droplets and solid particles from its vapor and 
liquid phases, respectively . Nucleation can be homogeneous as well as heterogeneous $[23,17,40]$. In homogeneous nucleation the new phase emerges due to the deposition of vapor on its own clusters, typically in the bulk of the domain, while heterogeneous nucleation occurs in the presence of 'foreign' substances which serve as deposition sites, either in the bulk of the domain or at its boundaries.

In this chapter, we present a computational method for studying homogeneous nucleation of droplets from a supersaturated vapor and their subsequent growth due to condensation. We consider gas-vapor two-phase laminar flow and initiate homogeneous nucleation by cooling down the hot gas carrying the vapor. Rapid cooling of the gas-vapor mixture results in a supersaturated state of the system and homogeneous nucleation occurs. Properties of the generated droplets, such as size and temperature, evolve depending on the local conditions in the flow domain. Relying on the classical nucleation theory $(\mathrm{CNT})[57,3]$ that provides explicit calculation of the nucleation rate as a function of supersaturation and temperature, we focus on developing a computational technique that allows a detailed investigation of the resulting aerosol evolution. We treat the gas-vapor mixture as a continuous phase, i.e., adopt an Eulerian description for it, while the droplets are modeled using the Lagrangian approach, i.e., the droplets are treated individually as dispersed point masses in the domain and their trajectories are explicitly computed in response to the flow in which the droplets are immersed $[34,5]$.

While most treatments of aerosol nucleation and growth via condensation and evaporation follow the Euler-Euler framework [17, 42, 26, 33], we developed an approach based on the Euler-Lagrange formulation, allowing a more 'microscopic' accounting of the various mechanisms at the scale of individual droplets. The nucleation rate which is key in the CNT is adopted in the Euler-Lagrange framework as the probability per unit of time and volume to generate a critical cluster. This link can be readily implemented in a computational model in which the local vapor concentration and temperature are used to determine the possible degree of supersaturation and correspondingly to evaluate the nucleation rate $J$. The number of critical clusters that could then be actually generated per grid cell of volume $\Delta V$ and per time step $\Delta t$ 
is then given by $\xi=J \Delta V \Delta t$. Well-resolved simulations imply $\xi \ll 1$, which is hence interpreted as the probability of actually generating such a cluster at a randomly chosen location in the grid cell.

We show results of simulation of flow in a simple channel and establish details of the nucleation process associated with rapid cooling, as well as subsequent growth of the droplets as these travel downstream. This illustrates a first application of the Euler-Lagrange approach to aerosol formation and presents aspects such as the evolving droplet size distribution and characteristics of the aerosol as it emanates from the end of the channel. It is a basis for studying the dependence of the aerosol formation process on important process parameters such as the temperature, the cooling rate and the flow velocity, which are subject of ongoing research.

The chapter is organized as follows. Section 4.2 is devoted to the mathematical modeling of two-phase gas-vapor flow coupled to nucleating liquid droplets. We start with the mathematical description of the gas-vapor flow. Afterwards, the governing equations for the generated liquid droplets are presented along with the coupling to the gas-vapor phase. Further computational methods used for solving the resulting system of equations are also described in this section. In Section 4.3 we apply the computational method to a channel flow between two parallel plates where we employ the classical theory of nucleation to simulate droplet formation in the Lagrangian setting. We conclude with a short summary of our findings in Section 4.4.

\subsection{Mathematical modeling}

In this section we discuss in detail the governing equations for the gas-vapor mixture and for the droplet phase. We also briefly describe the classical nucleation theory used to compute nucleation rates. Afterwards, we turn to the numerical methods used to solve the resulting system of equations. Throughout the chapter all the dimensional variables have the superscript ' ${ }^{*}$ ' while for the corresponding dimensionless variables the superscript is dropped. In the numerical experiment discussed in this chapter we consider a system of air and dibutylphthalate (DBT) vapor [40]. Further 
in discussions, we will refer to the air and vapor mixture as the carrier phase and to the dispersed DBT droplets as the liquid phase.

\subsubsection{Gas-vapor phase}

We start with describing flow, mass and heat transfer of the gas-vapor phase. We assume incompressible flow governed by the Navier-Stokes equations:

$$
\begin{aligned}
\nabla^{*} \cdot \mathbf{u}^{*} & =0 \\
\partial_{t}^{*}\left(\rho_{c}^{*} \mathbf{u}^{*}\right)+\mathbf{u}^{*} \cdot \nabla^{*}\left(\rho_{c}^{*} \mathbf{u}^{*}\right) & =-\nabla^{*} p^{*}+\nabla^{*} \cdot\left(\mu_{c}^{*} \nabla^{*} \mathbf{u}^{*}\right)
\end{aligned}
$$

with mass density of the carrier phase $\rho_{c}^{*}$ and dynamic viscosity $\mu_{c}^{*}$. This system of equations is solved for the unknown velocity $\mathbf{u}^{*}$ and pressure $p^{*}$. The governing equation for the vapor reads:

$$
\partial_{t}^{*}\left(\rho_{c}^{*} Y\right)+\mathbf{u}^{*} \cdot \nabla^{*}\left(\rho_{c}^{*} Y\right)=\nabla^{*} \cdot\left(\rho_{c}^{*} D^{*} \nabla^{*} Y\right)+M_{l \rightarrow v}^{*}
$$

where $Y$ is the vapor mass fraction, defined as:

$$
Y=\frac{\rho_{v}^{*}}{\rho_{c}^{*}}
$$

with $\rho_{v}^{*}$ the vapor mass density. While $\rho_{c}^{*}$ is considered constant in the domain, the vapor mass density may vary considerably as a result of nucleation and condensation processes. This approximation is justified in case of low vapor densities, i.e., in the situation that the carrier phase is predominantly made up of air. Moreover, $D^{*}$ is the diffusion coefficient of vapor and $M_{l \rightarrow v}^{*}$ is the rate at which mass is transferred from the liquid phase to the vapor phase due to the possible formation of droplets and evaporation/condensation. Finally, we consider advection-diffusion transport of the energy in terms of the temperature equation, given by:

$$
\rho_{c}^{*} c_{c}^{*}\left(\partial_{t}^{*} T_{c}^{*}+\mathbf{u}^{*} \cdot \nabla^{*} T_{c}^{*}\right)=\nabla^{*} \cdot\left(\lambda_{c}^{*} \nabla^{*} T_{c}^{*}\right)+S_{l \rightarrow v}^{*}
$$


where $c_{c}^{*}$ is the specific heat capacity of the carrier phase, $\lambda_{c}^{*}$ is the heat conductivity and $S_{l \rightarrow v}^{*}$ is the heat flow rate due to phase transitions.

\subsubsection{Droplet phase}

We consider the so-called Lagrangian description of the droplet phase. The motion of individual droplets is modeled, based on the simplified Maxey-Riley equations [34]. This approach is generalized to include diffusive motion of particles resulting from Brownian forcing. The trajectories of individual, spherical particles are obtained using the following system of equations:

$$
\frac{\mathrm{d} \mathbf{x}_{i}^{*}}{\mathrm{~d} t^{*}}=\mathbf{v}_{i}^{*}
$$

and

$$
\frac{\mathrm{d} \mathbf{v}_{i}^{*}}{\mathrm{~d} t^{*}}=\frac{3 \pi \mu_{c}^{*} d_{i}^{*}}{m_{i}^{*}}\left(\mathbf{u}_{i}^{*}-\mathbf{v}_{i}^{*}\right)+\mathbf{A}_{i}^{*}\left(t^{*}\right)
$$

where we use the subscript $i$ to indicate the properties of $i$-th droplet: $\mathbf{x}_{i}^{*}=\left\{x_{i}^{*}, y_{i}^{*}, z_{i}^{*}\right\}$ is the location vector of the droplet, $\mathbf{v}_{i}^{*}=\left\{u_{i}^{*}, v_{i}^{*}, w_{i}^{*}\right\}$ is the velocity vector of the droplet, $d_{i}^{*}$ is the diameter of the droplet, $m_{i}^{*}$ is its mass and $\mathbf{u}_{i}^{*}$ denotes the veloc-

ity of the carrier gas at particle position $\mathbf{x}_{i}^{*}$. Moreover, $\mathbf{A}_{i}^{*}=\left\{A_{x}^{*}, A_{y}^{*}, A_{z}^{*}\right\}$ is the random, time-dependent Brownian acceleration vector which is assumed to be independent of $\mathbf{v}_{i}^{*}$. It is assumed to fluctuate rapidly compared to the variations in $\mathbf{v}_{i}^{*}[5]$. Substituting $m_{i}^{*}=\pi \rho_{l}^{*}\left(d_{i}^{*}\right)^{3} / 6$ we get:

$$
\frac{\mathrm{d} \mathbf{v}_{i}^{*}}{\mathrm{~d} t^{*}}=\frac{\mathbf{u}_{i}^{*}-\mathbf{v}_{i}^{*}}{\tau_{v, i}^{*}}+\mathbf{A}_{i}^{*}\left(t^{*}\right)
$$

with relaxation time

$$
\tau_{v, i}^{*}=\frac{C_{c} \rho_{l}^{*}\left(d_{i}^{*}\right)^{2}}{18 \mu_{c}^{*}}
$$


where $\rho_{l}^{*}$ is the density of the liquid droplets. The Cunningham correction factor is essential when calculating the drag on small particles to account for non-continuum effects and is given by [8]: .

$$
C_{c}=1+\frac{2 \lambda^{*}}{d_{p}^{*}}\left(1.257+0.4 \exp \left(\frac{-1.1 d_{p}^{*}}{2 \lambda^{*}}\right)\right)
$$

where $\lambda^{*}$ is the free mean path of DBT vapor. The model for the Brownian accelerations is identical to that used in the previous Chapter.

Conservation of total mass around a single spherical particle leads to the following equation for the particle's mass change due to evaporation and condensation [7]:

$$
\frac{\mathrm{d} m_{i}^{*}}{\mathrm{~d} t^{*}}=-\left(\frac{m_{i}^{*} S h_{i}}{3 \tau_{v, i}^{*} S c}\right) \ln \left(\frac{1-Y_{\delta}}{1-Y_{0}}\right)
$$

where $S c=\mu_{c}^{*} /\left(\rho_{c}^{*} D^{*}\right)$ is the Schmidt number and $S h_{i}=2+0.552 R e_{r, i}^{1 / 2} S c^{1 / 3}$ is the Sherwood number. The Reynolds number of the motion of the droplet relative to the surrounding fluid is defined as $R e_{r, i}=\left(d_{i}^{*}\left|\mathbf{u}\left(\mathbf{x}_{i}^{*}\right)-\mathbf{v}_{i}^{*}\right|\right) / \nu_{c}^{*}$ where $\nu_{c}^{*}=\mu_{c}^{*} / \rho_{c}^{*}$ is the kinematic viscosity of the carrier phase. The vapor mass fraction $Y_{\delta}$ stands for the vapor mass fraction at distance $\delta$ from the surface of the droplet while $Y_{0}$ is the vapor mass fraction at the surface of the droplet. We compute $Y_{\delta}$ by interpolation of the vapor mass fraction field $Y$ to the droplet location. The saturation mass fraction $Y_{0}$ at a given temperature $T^{*}$ for the DBT vapor is obtained from its constitutive relation [40]:

$$
Y_{0}=\exp \left(21.497-11497 / T^{*}\right)
$$

Considering convective heat transfer, the energy equation can be written as [7]:

$$
m_{i}^{*} c_{l}^{*} \frac{\mathrm{d} T_{i}^{*}}{\mathrm{~d} t^{*}}=\left(h_{v}^{*}-h_{l}^{*}\right) \frac{\mathrm{d} m_{i}^{*}}{\mathrm{~d} t^{*}}+N u_{i} \pi \lambda_{c}^{*} d_{i}^{*}\left(T_{c, i}^{*}-T_{i}^{*}\right)
$$

where the Nusselt number is given by $N u_{i}=2+0.6 \operatorname{Re}_{r, i}^{1 / 2} \operatorname{Pr}^{1 / 3}$ in terms of the relative Reynolds number $R e_{r, i}$ and the Prandtl number $\operatorname{Pr}$ that will be specified momentarily in connection with the selected reference scales for the non-dimensionalization. In 
addition, $h_{v}^{*}$ and $h_{l}^{*}$ are the specific enthalpy of the vapor and the droplet, respectively. The enthalpy difference $\left(h_{v}^{*}-h_{l}^{*}\right)$ is the enthalpy associated with the change of phase at the droplet temperature, i.e., the latent heat of evaporation. The second term in the right hand side of (4.13) denotes conductive the heat transfer. We can rewrite (4.13):

$$
\frac{\mathrm{d} T_{i}^{*}}{\mathrm{~d} t^{*}}=\frac{\left(h_{v}^{*}-h_{l}^{*}\right)}{m_{i}^{*} c_{l}^{*}} \frac{\mathrm{d} m_{i}^{*}}{\mathrm{~d} t^{*}}+\frac{N u}{2} \frac{1}{\tau_{T, i}^{*}}\left(T_{c, i}^{*}-T_{i}^{*}\right)
$$

with $\tau_{T, i}^{*}$ the thermal response time, defined as:

$$
\tau_{T, i}^{*}=\frac{c_{l}^{*} \rho_{l}^{*}\left(d_{i}^{*}\right)^{2}}{12 \lambda_{c}^{*}}
$$

Apart from the motion of the droplets and their response in terms of mass and temperature, it is essential to actually generate droplets. In order to nucleate a droplet the so-called saturation $S_{r}$ should be sufficiently larger than unity. This saturation is the ratio of the partial pressure of the vapor $P_{v, p a r}^{*}$ in the gas-vapor mixture to the saturation vapor pressure at the temperature of the mixture:

$$
S_{r}=\frac{P_{v, p a r}^{*}}{P_{v, s a t}^{*}}
$$

When the saturation ratio is greater than 1, the gas-vapor mixture is supersaturated, when the ratio equals 1 , then the mixture is saturated and if the saturation ratio is less than 1 , then the mixture is unsaturated. The partial vapor pressure $P_{v, p a r}^{*}$ is computed using the ideal gas law [54]:

$$
P_{v, p a r}^{*}=\frac{k_{B}^{*} T^{*} \rho_{v}^{*} Y}{M^{*}}
$$

where $T^{*}$ and $Y$ are to be evaluated at the location of the droplet via interpolation. Moreover, $k_{B}^{*}=1.3806488 \cdot 10^{-23}\left(\mathrm{~m}^{2} \cdot \mathrm{kg}\right) /\left(\mathrm{s}^{2} \cdot K\right)$ denotes the Boltzmann's constant and $M^{*}$ is the molecular mass of the vapor. We use (4.12) and the ideal gas law to compute the corresponding saturation vapor pressure at the local temperature $T^{*}$. 
Summarizing we get the following system of equations:

$$
\begin{aligned}
\nabla^{*} \cdot \mathbf{u}^{*} & =0 \\
\frac{\partial^{*}\left(\rho_{c}^{*} \mathbf{u}^{*}\right)}{\partial t}+\mathbf{u}^{*} \cdot \nabla^{*}\left(\rho_{c}^{*} \mathbf{u}^{*}\right) & =-\nabla^{*} p^{*}+\nabla^{*} \cdot\left(\mu_{c}^{*} \nabla^{*} \mathbf{u}^{*}\right) \\
\rho_{c}^{*} c_{c}^{*}\left(\partial_{t}^{*} T_{c}^{*}+\mathbf{u}^{*} \cdot \nabla^{*} T_{c}^{*}\right) & =\nabla^{*} \cdot\left(\lambda_{c}^{*} \nabla^{*} T_{c}^{*}\right)+S_{l \rightarrow v}^{*} \\
\partial_{t}^{*}\left(\rho_{c}^{*} Y\right)+\mathbf{u}^{*} \cdot \nabla^{*}\left(\rho_{c}^{*} Y\right) & =\nabla^{*} \cdot\left(\rho_{c}^{*} D^{*} \nabla^{*}(Y)\right)+M_{l \rightarrow v}^{*} \\
\frac{\mathrm{d} \mathbf{x}_{i}^{*}}{\mathrm{~d} t^{*}} & =\mathbf{v}_{i}^{*} \\
\frac{\mathrm{d} \mathbf{v}_{i}^{*}}{\mathrm{~d} t^{*}} & =\frac{\mathbf{u}_{i}^{*}-\mathbf{v}_{i}^{*}}{\tau_{v, i}^{*}}+\mathbf{A}_{i}^{*}\left(t^{*}\right) \\
\frac{\mathrm{d} m_{i}^{*}}{\mathrm{~d} t^{*}} & =-\left(\frac{m_{i}^{*} S h_{i}}{3 \tau_{v, i}^{*} S c}\right) \ln \left(\frac{1-Y_{\delta}}{1-Y_{0}}\right) \\
\frac{\mathrm{d} T_{i}^{*}}{\mathrm{~d} t^{*}} & =\frac{\left(h_{v}^{*}-h_{l}^{*}\right)}{m_{i}^{*} c_{l}^{*}} \frac{\mathrm{d} m_{i}^{*}}{\mathrm{~d} t^{*}}+\frac{N u}{2} \frac{1}{\tau_{T, i}^{*}}\left(T_{c, i}^{*}-T_{i}^{*}\right)
\end{aligned}
$$

The following scaling is applied to the dimensional formulation to obtain the final non-dimensional formulation of (4.18) - (4.25).

$$
\begin{array}{rlr}
u^{*}=U_{r e f}^{*} u, & x^{*}=L_{r e f}^{*} x, & t^{*}=t_{r e f}^{*} t \\
p^{*}=p_{r e f}^{*} p, \quad T_{c}^{*}=T_{r e f}^{*} T_{c}, & \rho_{c}^{*}=\rho_{r e f}^{*} \rho_{c}, \\
\mu_{c}^{*}=\mu_{r e f}^{*} \mu_{c}, & c_{c}^{*}=c_{r e f}^{*} c_{c}, & \lambda_{c}^{*}=\lambda_{r e f}^{*} \lambda_{c} \\
S_{l \rightarrow v}^{*}=S_{r e f}^{*} S_{l \rightarrow v}, & M_{l \rightarrow v}^{*}=M_{r e f}^{*} M_{l \rightarrow v}, & D^{*}=D_{r e f}^{*} D
\end{array}
$$


The resulting non-dimensional formulation reads:

$$
\begin{aligned}
\nabla \cdot \mathbf{u} & =0 \\
\frac{\partial\left(\rho_{c} \mathbf{u}\right)}{\partial t}+\mathbf{u} \cdot \nabla\left(\rho_{c} \mathbf{u}\right) & =-\nabla p+\frac{1}{R e} \nabla \cdot\left(\mu_{c} \nabla \mathbf{u}\right) \\
\rho_{c} c_{c}\left(\partial_{t} T_{c}+\mathbf{u} \cdot \nabla T_{c}\right) & =\frac{1}{R e} \frac{1}{P r} \nabla \cdot\left(\lambda_{c} \nabla T_{c}\right)+S_{l \rightarrow v} \\
\partial_{t}\left(\rho_{c} Y\right)+\mathbf{u} \cdot \nabla\left(\rho_{c} Y\right) & =\nabla \cdot\left(\rho_{c} D \nabla Y\right)+M_{l \rightarrow v} \\
\frac{\mathrm{d} \mathbf{x}_{i}}{\mathrm{~d} t} & =\mathbf{v}_{i} \\
\frac{\mathrm{d} \mathbf{v}_{i}}{\mathrm{~d} t} & =\frac{\mathbf{u}_{i}-\mathbf{v}_{i}}{\tau_{v, i}}+\mathbf{A}_{i}(t) \\
\frac{\mathrm{d} m_{i}}{\mathrm{~d} t} & =-\left(\frac{m_{i} S h_{i}}{3 \tau_{v, i} S c}\right) \ln \left(\frac{1-Y_{\delta}}{1-Y_{0}}\right) \\
\frac{\mathrm{d} T_{i}}{\mathrm{~d} t} & =E \frac{\left(h_{v}-h_{l}\right)}{m_{i} c_{l}} \frac{\mathrm{d} m_{i}}{\mathrm{~d} t}+\frac{N u}{2} \frac{1}{\tau_{T, i}}\left(T_{c, i}-T_{i}\right)
\end{aligned}
$$

where the several reference scales are interrelated through the following relations:

$$
\begin{aligned}
& \frac{U_{r e f}^{*} t_{r e f}^{*}}{L_{r e f}^{*}}=1, \quad p_{r e f}^{*}=\rho_{r e f}^{*}\left(U_{r e f}^{*}\right)^{2} \\
& \frac{D_{r e f}^{*} t_{r e f}^{*}}{\left(L_{r e f}^{*}\right)^{2}}=1, \quad M_{r e f}^{*}=\frac{\rho_{r e f}^{*}}{t_{r e f}^{*}}, \\
S_{r e f}^{*}= & \frac{\rho_{r e f}^{*} c_{r e f}^{*} T_{r e f}^{*}}{t_{r e f}^{*}}
\end{aligned}
$$

The non-dimensional parameters $\operatorname{Re}, \operatorname{Pr}, \tau_{v, i}, \tau_{t, i}$ and $E$ are defined as:

$$
\begin{gathered}
R e=\frac{\rho_{r e f}^{*} U_{r e f}^{*} L_{r e f}^{*}}{\mu_{r e f}^{*}}, \quad \operatorname{Pr}=\frac{c_{r e f}^{*} \mu_{r e f}^{*}}{\lambda_{r e f}^{*}} \\
\tau_{v, i}=\tau_{v, i}^{*} / t_{r e f}^{*}, \quad \tau_{T, i}=\tau_{T, i}^{*} / t_{r e f}^{*} \\
E=\frac{h_{r e f}^{*}}{T_{r e f}^{*} c_{r e f}^{*}}
\end{gathered}
$$

Finally, the dimensionless mass $m_{i}$ is related to the actual mass of a droplet $m_{i}^{*}$ using as unit of mass the weight of the critical cluster $m_{n u t}^{*}$ as will be specified in the next subsection.

In the next subsection we describe in more details the source terms in the above 
system of equations.

\subsubsection{Coupling the discrete and the continuous phases}

Due to the fluid-droplet interaction mass and energy transfer between the phases occurs expressed by the source terms in the temperature and vapor mass fraction equations for the carrier phase. Below we describe these source terms.

We start with the mass flow rate from the liquid to the vapor phase $M_{l \rightarrow v}^{*}$ per unit volume. This contains two contributions: the nucleation mass flow rate $M_{n u c}^{*}$ and the mass flow rate due to evaporation minus that due to condensation $M_{e-c}^{*}$.

$$
M_{l \rightarrow v}^{*}=-M_{n u c}^{*}+M_{e-c}^{*}
$$

The source term expressing the mass coupling due to evaporation/condensation $M_{e-c}^{*}$ accounts for the local change in vapor concentration field due to evaporation/condensation:

$$
M_{e-c}^{*}=-\sum_{i} \frac{\mathrm{d} m_{i}^{*}}{\mathrm{~d} t^{*}} \delta\left(\mathbf{x}-\mathbf{x}_{i}\right)
$$

Here $\delta$ is the Dirac delta-function. The nucleation mass rate per unit volume is specified as the product of the mass of a droplet at nucleation and the nucleation (formation) rate $J_{N}^{*}$ :

$$
M_{n u c}^{*}=m_{n u c}^{*} J_{N}^{*}
$$

Considerable literature is available providing expressions for the nucleation rate as a function of supersaturation and temperature. Here, we employ the classical theory which allows to compute nucleation rates in terms of local or microscopic properties.

The mass of a droplet at nucleation is related to the diameter $d_{n u c}^{*}$ of the nucleated droplets, which is the diameter of the so-called 'critical' cluster. A nucleus with a critical diameter is in unstable equilibrium with the surrounding vapor having an equal probability of $50 \%$ for growth and for disappearance. It is common to adopt a 'safety margin' for the diameter of the droplets at nucleation that is larger by a factor of $2^{1 / 3}$, resulting in a twice as large mass of nucleated droplets [23]. The critical 
diameter is specified as:

$$
d_{\text {nuc }}^{*}=2^{1 / 3} \frac{4 \sigma^{*} V_{m}^{*}}{\ln \left(S_{r}\right) k_{B}^{*} T^{*}}
$$

with $\sigma^{*}$ the surface tension of the critical cluster droplet, assuming that the critical cluster has the same properties as a macroscopic liquid drop. Moreover, $V_{m}^{*}$ the molecular volume, i.e, the volume occupied by one molecule of the vapor. The mass of a droplet at nucleation is then computed as:

$$
m_{n u c}^{*}=\frac{\pi\left(d_{n u c}^{*}\right)^{3} \rho_{l}^{*}}{6}
$$

According to the classical theory of nucleation, the nucleation rate $J_{N}^{*}$ in a supersaturated state depends on the temperature and the supersaturation as follows:

$$
J_{N}^{*}= \begin{cases}\gamma^{*} Y^{2} \exp \left(-\alpha \ln ^{-2}\left(S_{r}\right)\right), & S_{r}>1 \\ 0, & S_{r} \leq 1\end{cases}
$$

with combined 'material' parameters

$$
\gamma^{*}=\sqrt{\frac{2 \sigma^{*}}{\pi M^{*}}} V_{m}^{*}\left(\frac{\rho_{c}^{*}}{M^{*}}\right)^{2}
$$

and

$$
\alpha=\frac{16 \pi\left(V_{m}^{*}\right)^{2}\left(\sigma^{*}\right)^{3}}{3\left(k_{B}^{*} T^{*}\right)^{3}}
$$

The surface tension of the fluid-vapor interface depends on the temperature:

$$
\sigma^{*}=\frac{\kappa^{*}\left(T_{c r}^{*}-T^{*}\right)}{\left(V_{m o l}^{*}\right)^{2 / 3}}
$$

with $\kappa^{*}=2.1 \times 10^{-7} \mathrm{~J} \cdot K^{-1} \mathrm{~mol}^{-2 / 3}$ a constant valid for many liquids, $V_{m o l}^{*}$ the molar mass of DBT vapor, $T_{c r}^{*}$ the critical temperature for which the surface tension reaches the value of zero. For DBT we use $\sigma^{*}=0.001\left(35.3-0.0863\left(T^{*}-273.15\right)\right) \mathrm{J} \cdot \mathrm{m}^{-2}$ [40]. For an arbitrary volume and in an arbitrary time interval the total number of 
nucleated droplets is:

$$
N=\int_{\Delta V^{*}} d V^{*} \int_{\Delta t^{*}} d t^{*} J_{N}^{*}
$$

If we specify this to an elementary volume $\Delta V^{*}$ and time interval $\Delta t^{*}$, then:

$$
N=\Delta t^{*} \Delta V^{*} J_{N}^{*}
$$

This is a crucial connection in order to translate the nucleation rate to its consequence in the Euler-Lagrangian framework.

Similar to the case of mass flux, the total heat flow rate $S_{l \rightarrow v}^{*}$ is composed of two components: heat flow rate due to nucleation $S_{n u c}^{*}$ and heat flow rate due to evaporation and condensation $S_{e-c}^{*}$

$$
S_{l \rightarrow v}^{*}=S_{n u c}^{*}-S_{e-c}^{*}
$$

The heat flow rate due to nucleation is related to the mass flow rate $M_{n u c}^{*}$ using the enthalpy of vaporization $\Delta H^{*}$ in the following way:

$$
S_{n u c}^{*}=\Delta H^{*} M_{n u c}^{*}
$$

The heat rate due to evaporation and condensation is given by:

$$
S_{e-c}^{*}=\sum_{i}\left(c_{l}^{*} \frac{\mathrm{d}}{\mathrm{d} t^{*}}\left(m_{i}^{*} T_{i}^{*}\right)\right) \delta\left(\mathbf{x}-\mathbf{x}_{i}\right)=\sum_{i}\left(c_{l}^{*} m_{i}^{*} \frac{\mathrm{d} T_{i}^{*}}{\mathrm{~d} t^{*}}+c_{l}^{*} T_{i}^{*} \frac{\mathrm{d} m_{i}^{*}}{\mathrm{~d} t^{*}}\right) \delta\left(\mathbf{x}-\mathbf{x}_{i}\right)
$$

Since we follow the trajectories of the droplets in detail as well as the evolution of their mass and temperature, all terms in this expression are directly available from the dispersed phase description. Recalling the definition of $S_{r e f}^{*}$, this leads to the contribution $S_{e-c}=\frac{S_{e-c}^{*}}{S_{r e f}^{*}}$ to the source term $S_{l \rightarrow v}$ :

$$
S_{e-c}=\sum_{i}\left(c_{l} m_{i} \frac{\mathrm{d} T_{i}}{\mathrm{~d} t}+c_{l} T_{i} \frac{\mathrm{d} m_{i}}{\mathrm{~d} t}\right) \delta\left(\mathbf{x}-\mathbf{x}_{i}\right)
$$


Having described the set of equations next we turn to the numerical approaches applied for solving it.

\subsubsection{Numerical treatment}

The system of equations formulated in the previous sections is solved numerically. Below we address the main numerical schemes and methods that were adopted.

We first consider the numerical solution of the Navier-Stokes equations. For the simulation of incompressible flow a finite-volume discretization of the equations (4.30) - (4.31) is used. Here, we consider a staggered storage of variables, which helps avoiding unphysical pressure fields that might result from the pressure-velocity coupling step in solving the Navier-Stokes equations. In the 3D case the scalar variables, such as pressure, are stored in the middle of the grid cell, while the velocity components are stored in the center of the corresponding face of the grid cell.

Discretization of the Navier-Stokes equations requires approximation of the differential operators appearing in the equations. The skew-symmetry of the differential operators $(\mathbf{u} \cdot \nabla)$ and $\nabla$ implies that the total energy of the flow is conserved when the flow is inviscid. It only decreases when there is dissipation. To achieve this on the discrete level a discrete skew-symmetric approximation of the operator $(\mathbf{u} \cdot \nabla)$ and a positive-definite approximation of $-\nabla \cdot \nabla$ are developed in [56] which we employ in the simulations presented in the next Section.

We next discuss the method to simulate the motion of the droplets. For sake of simplicity, we drop the subscript ' $i$ ' whenever referring to the $i$-th droplet. Given the droplet's position and velocity at time $t^{n-1}$ its velocity at $t^{n}=t^{n-1}+\Delta t$ is computed using the model for the Brownian motion as specified in the previous Chapter. For the $x$-component of the motion this implies:

$$
\begin{aligned}
v^{n} & =v^{n-1} \exp \left(-\Delta t / \tau_{v}^{n-1}\right)+u_{x}\left(\mathbf{x}^{n-1}\right)\left(1-\exp \left(-\Delta t / \tau_{v}^{n-1}\right)\right) \\
& +\sqrt{\frac{k_{B}^{*} T_{r e f}^{*} T\left(\mathbf{x}^{n-1}\right)}{m_{r e f}^{*} m^{n-1} U_{r e f}^{*}}} \sqrt{1-\exp \left(-2 \Delta t / \tau_{v}^{n-1}\right)} g^{n}
\end{aligned}
$$


with $g^{n}$ a random number drawn from $N(0,1)$. The Stokes number is time-dependent because of the changing mass of the droplet in the course of time. It is equal to the droplet relaxation time according to its actual instantaneous weight, made dimensionless by the reference time-scale. For the Brownian part of this model to be valid, the time step $\Delta t$ is assumed to be large compared to the expectation value for the length of the time interval between successive collisions of a droplet with the gas molecules. This implies that we only incorporate the 'average' motion due to accumulated effects of the Brownian motion due to molecular agitation. On the other hand, the time step is much smaller than the particle relaxation time. This implies, that $\Delta t / \tau_{v}^{n} \ll 1$. For this limit, by approximating $\exp \left(-\Delta t / \tau_{v}^{n}\right)=1-\Delta t / \tau_{v}^{n}$, we can rewrite (4.59) in the form of an Euler-forward scheme, which we also apply to compute particle's position, mass and temperature. For the $x$-direction this is:

$$
\left\{\begin{aligned}
x^{n} & =x^{n-1}+\Delta t v_{x}^{n-1} \\
v^{n} & =v^{n-1}\left(1-\Delta t / \tau_{v}^{n-1}\right)+u_{x}\left(\mathbf{x}^{n-1}\right) \Delta t / \tau_{v}^{n-1}+\sqrt{\frac{2 \Delta t k_{B}^{*} T_{r e f}^{*} T\left(\mathbf{x}^{n-1}\right)}{m_{r e f}^{*} m^{n-1} U_{r e f}^{*} \tau_{v}^{n-1}}} g^{n} \\
m^{n} & =m^{n-1}\left(1-\frac{\Delta t S h_{n}}{3 \tau_{v}^{n-1} S c} \ln \left(\frac{1-Y_{\delta}}{1-Y_{0}}\right)\right) \\
T^{n} & =T^{n-1}+E \frac{\left(h_{v}-h_{l}\right)}{m^{n-1} c_{l}}\left(m^{n}-m^{n-1}\right)+\frac{\Delta t N u}{2} \frac{1}{\tau_{T}^{n-1}}\left(T_{c}-T^{n-1}\right)
\end{aligned}\right.
$$

In order to propagate the particle position, velocity, mass and temperature over a time step the velocity of the gas, vapor mass concentration and temperature at the particle position have to be computed. This requires interpolation of latter fields at the particle position. We use trilinear interpolation.

\subsection{Droplet formation and growth in a channel flow}

In this section we consider a numerical experiment to demonstrate the above developed computational technique to study aerosol droplet formation and evolution. We consider an air-DBT vapor mixture flowing at a constant rate between two parallel 


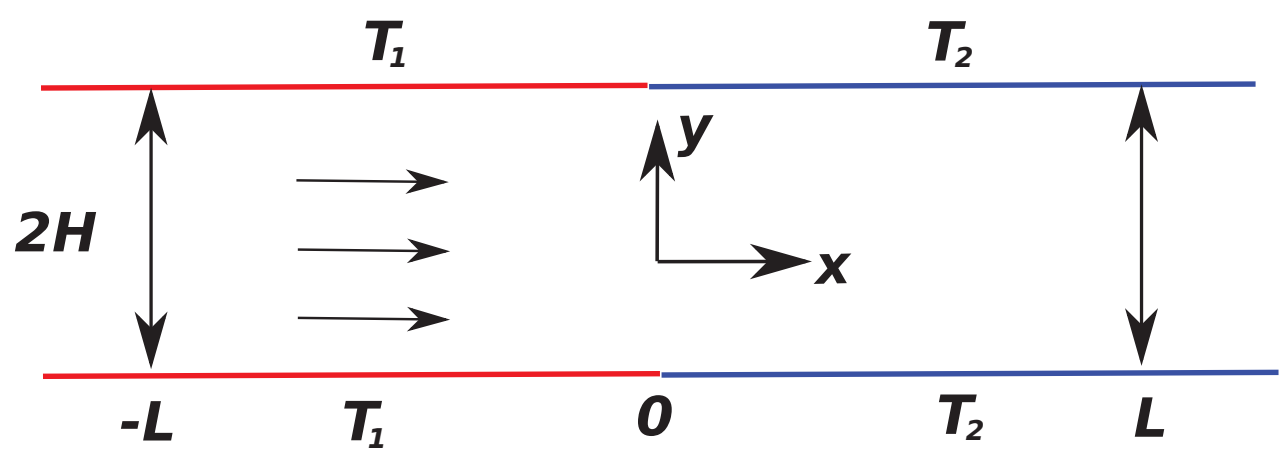

Figure 4-1: Two parallel plates forming a channel. The temperature of the plates at the left of the origin is $T_{1}$ and to the right of the origin is $T_{2}$. The inflow boundary is at the left at $x=-L$ at which gas and vapor enter at temperature $T_{1}$ and vapor mass fraction $Y_{0}$ which yields an unsaturated condition at the inflow. At the outflow a convective outflow boundary condition is adopted while at the walls Dirichlet conditions for the temperature are imposed, no-slip conditions for the velocity and no-penetration Neumann conditions for the vapor.

plates. We initiate homogeneous nucleation of droplets by cooling down the hot gas mixture. Under suitable process conditions, rapid cooling of the gas-vapor mixture results in a supersaturated state of the system and homogeneous nucleation may occur. We will illustrate this process with simulations based our our model and concentrate on temperature and size distributions of the aerosol droplets which move in the flow and propagate through the channel while undergoing condensation/evaporation.

\subsubsection{Numerical experiment}

The numerical experiment requires two steps. First, we set up a 'pre-cursor simulation' without any vapor, in which we compute the fully developed laminar Poiseuille flow through the channel subject to a uniform temperature $T_{1}$ at the inflow and at the walls. Subsequently, a temperature drop in the channel is applied through the boundary conditions at the walls of the channel. In particular, the right half $x>0$ of the channel wall is given a temperature $T_{2}<T_{1}$ (see Figure 4-1). At the same time, at the inlet of the channel DBT vapor is introduced into the system at constant mass fraction. The aim is to follow the development of the temperature, vapor and droplet phase as the system evolves, following the imposition of the temperature drop.

The pre-cursor simulation for the velocity uses no-slip boundary conditions at the 
walls and periodic conditions in the stream-wise $x$-direction. Moreover, to maintain a fixed mass flow through the domain a constant pressure gradient is added to the momentum equation in the $x$-direction. The flow in the wall-normal $y$ and the spanwise $z$ directions is set to zero. The flow is simulated until a well-developed steady state is obtained which compares well with Poiseuille flow. For this we monitored the residual for the stream-wise velocity and continued the simulation until the residual no longer decreases. The Reynolds number $R e=100$ of the computed flow is based on the length and velocity scales $L_{r e f}^{*}=2 H=0.01 \mathrm{~m}$ and $U_{r e f}^{*}=0.21 \mathrm{~m} / \mathrm{s}$. We adopt constant values for $S c=3.8, S h=2.8$ and $N u=2.6$ considering air properties at $T=100 C$, namely, $\rho_{c}^{*}=0.946 \mathrm{~kg} / \mathrm{m}^{3}, \mu_{c}^{*}=2.2 \times 10^{-5} \mathrm{~kg} /(\mathrm{m} \cdot \mathrm{s})$. For the vapor diffusivity we use $D^{*}=0.0398(T / 273.16)^{3 / 2} \mathrm{~cm}^{2} / \mathrm{s}$ while the DBT liquid density is computed from $\rho_{l}^{*}=1.063-0.000826\left(T^{*}-273.16\right) \mathrm{g} \mathrm{cm}^{-3}$. Moreover, for the dimensionless Prandtl number we assume $\operatorname{Pr}=1$.

The problem for the temperature and vapor mass fraction is two-dimensional, the initial and boundary conditions for the temperature can be expressed as follows:

$$
\begin{aligned}
& T(x, y, 0)=T_{1}, \quad-L<x<L,-H<y<H \\
& T(-L, y, t)=T_{1}, \quad-H<y<H, t>0 \\
& \partial_{x} T(L, y, t)=0, \quad-H<y<H, t>0
\end{aligned}
$$

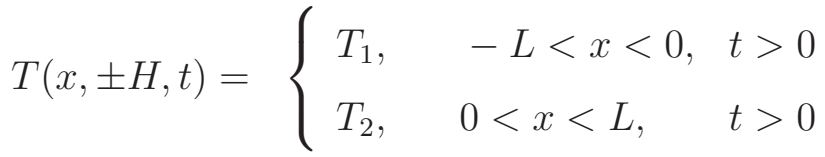

i.e., initially the temperature is set at $T_{1}$, which is maintained throughout at the inflow and in the first half $(-L<x<0)$ at the walls of the flow domain. In the second half $(L>x>0)$ the walls are kept at $T_{2}<T_{1}$ while at the outflow the solution is approximated as independent of the stream-wise coordinate. For the vapor mass concentration we assume initially a situation without any vapor. At the inflow, vapor can enter at a value $Y_{i n}$, which can not leave the domain through the walls. Finally, near the outflow, the solution is assumed independent of the stream-wise coordinate. 


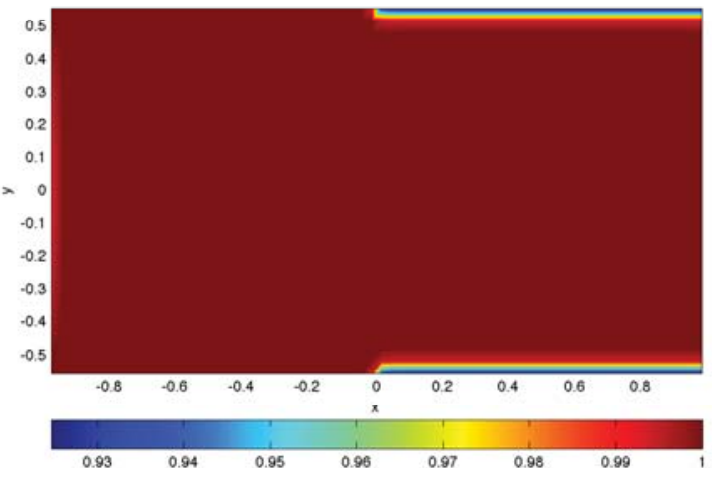

(a) $t=0.0002$

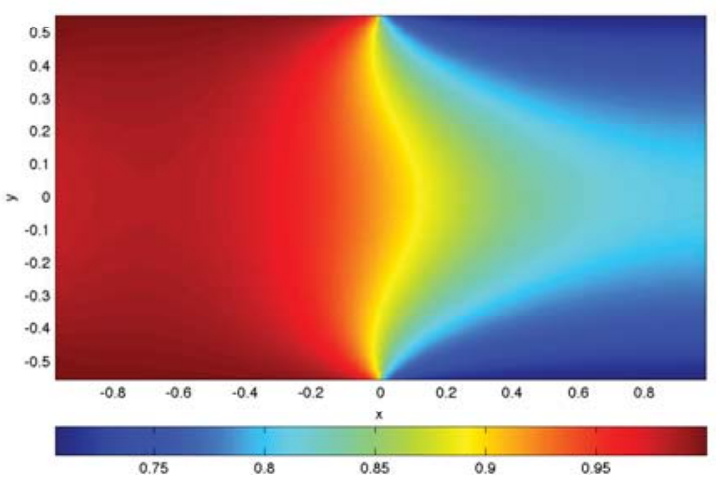

(c) $t=0.17$

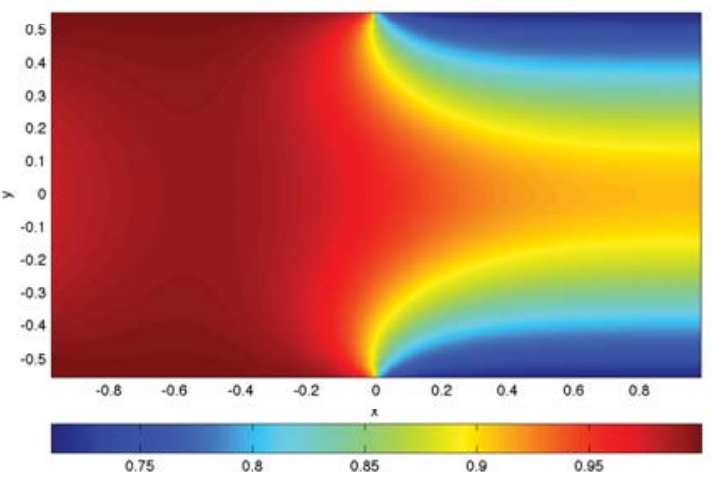

(b) $t=0.07$

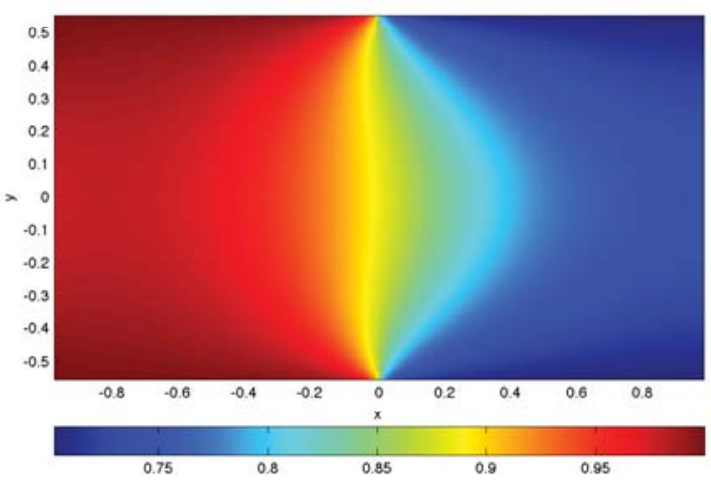

(d) $t=0.58$

Figure 4-2: Evolution of the temperature distribution at $T_{1}=1$ and $T_{2}=0.7$ computed at resolution of $\{64 \times 32\}$. The situation at $t=0.58$ is a good approximation of the steady solution.

Mathematically, this is expressed by:

$$
\begin{array}{llll}
Y(x, y, 0) & =0, \quad-L<x<L,-H<y<H & \\
Y(-L, y, t) & =Y_{\text {in }}, & -H<y<H, & t>0 \\
\partial_{x} Y(L, y, t) & =0, & & t+H<y<H, \\
& & & t>0 \\
\partial_{y} Y(x, \pm H, t)=0, & -L<x<L, & t>0
\end{array}
$$

Figure 4-2 shows the evolution of the temperature field in response to the imposed temperature drop for $t>0$ at the walls in the second half of the domain, i.e., $0<$ 


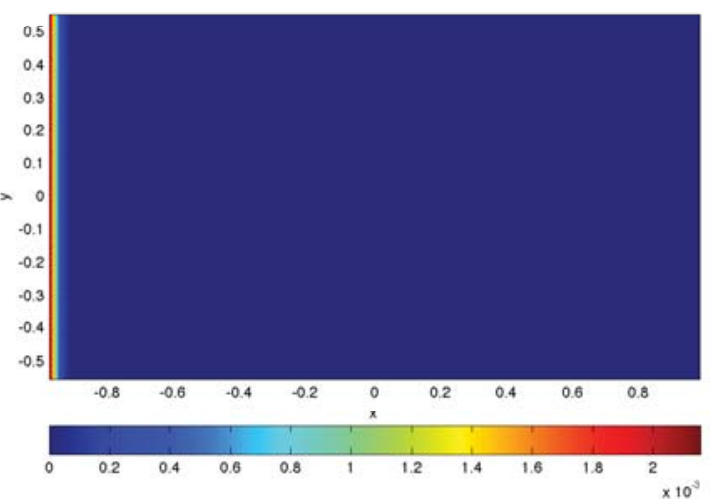

(a) $t=0$

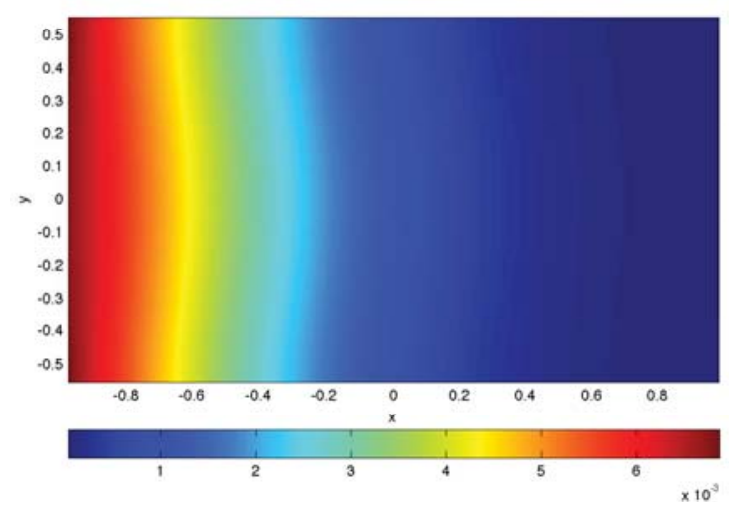

(c) $t=0.17$

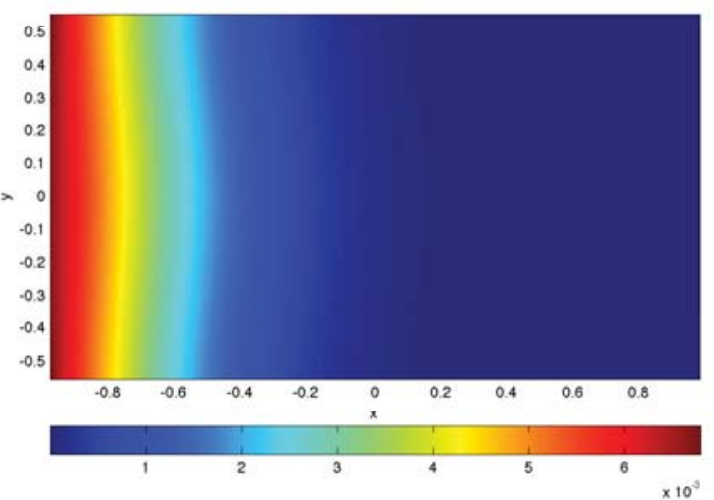

(b) $t=0.07$

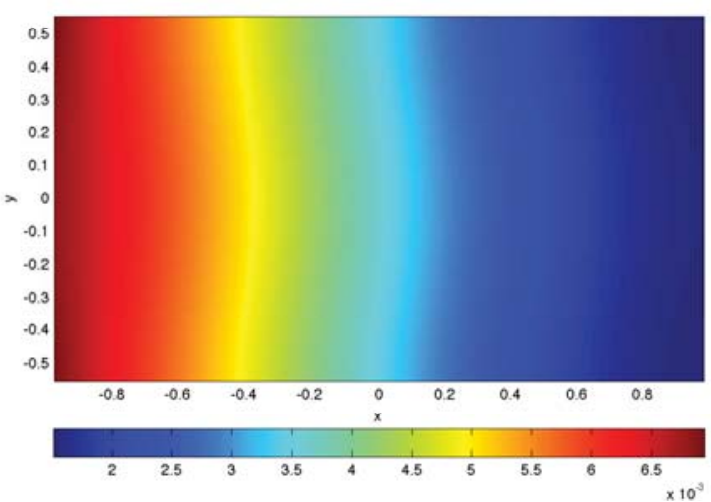

(d) $t=0.58$

Figure 4-3: Vapor mass distribution at different simulation times corresponding to an inflow vapor mass fraction $Y_{\text {in }}=7 \times 10^{-3}$ computed at resolution of $\{64 \times 32\}$.

$x<L$. The temperature contrast used in this illustration is $T_{1}=1$ and $T_{2}=0.7$. As a result of this cooling we observe a gradual establishment of a characteristic temperature profile where during the earlier stages 'fronts' of colder temperature diffuse into the domain and establish a smooth temperature distribution with a rather sharp gradient in the stream-wise direction around $x=0$.

Figure 4-3 shows the evolution of the vapor mass fraction. The gas-vapor mixture is allowed to flow into a domain that initially does not contain any vapor. The vapor is seen to penetrate smoothly into the domain, carried by the flow and occupies the domain. is cooled by the walls as the vapor gradually propagates through the channel.

As the vapor propagates into the colder regions of the channel the saturation builds up and at some moment small areas of supersaturated vapor emerge. This initiates 
the nucleation of droplets, which subsequently are carried further downstream by the flow. As a result of condensation of vapor onto the droplets their sizes will change. This will be considered in the following subsection.

\subsubsection{Droplet generation and growth}

We focus on the size and temperature distribution of the droplets in the domain and as they exit at the outflow. In realistic cases a very large number of droplets can form, which would be impossible to handle with the current algorithm. Therefore, we selected a case in which nucleation is not quite as strong and considered the generation of up to $10^{4}$ droplets. Once this number was reached, no further nucleation was allowed and this 'puff' of droplets was tracked in the domain while their properties were allowed to evolve. The evolution of droplet properties in such a limited puff approximates the evolution of a realistic number of droplets since we consider only rather low concentrations in which flow and dispersed phase are only mildly coupled.

At each time step the probability to generate a droplet in a grid cell of volume $\Delta V$ is computed using (4.54). A droplet with diameter $d_{n u c}^{*}$, given by (4.47), may then be generated in that cell. The droplet is initialized at a random location within the grid cell. The initial velocity and temperature of the droplet are taken as the gas velocity and temperature at the droplet's location. We adhere to well-resolved situations in which the probability to actually generate a new droplet in a cell is well below unity, implying the use of sufficiently small time steps in order to capture the first stages of nucleation.

After the nucleation, the position, velocity, mass and temperature of the individual droplets are tracked as a function of time. For this we use (4.60). Results in this section are based on $T_{\text {ref }}^{*}=393 K(120 C)$ and $Y_{i n}=7 \cdot 10^{-3}$. In Table 4.3.2 properties of DBT vapor are summarized which are used to compute the nucleation rate $J_{N}^{*}$.

Snapshots of droplet locations are shown in Figure 4-4. The majority of the droplets are formed in the near wall region where cooling leads to the highest super- 


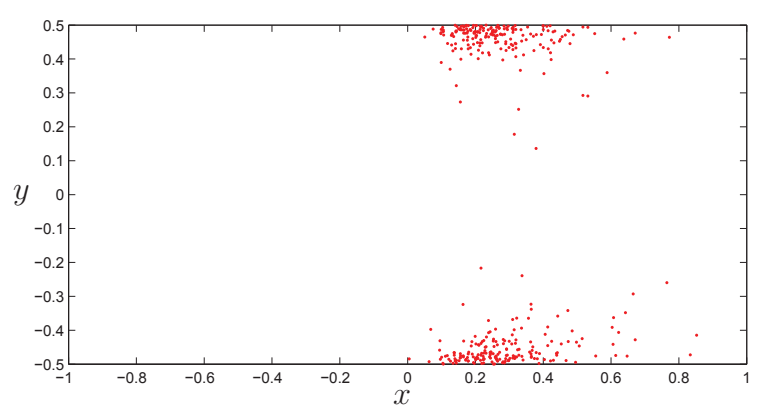

(a) $t=0.58005$

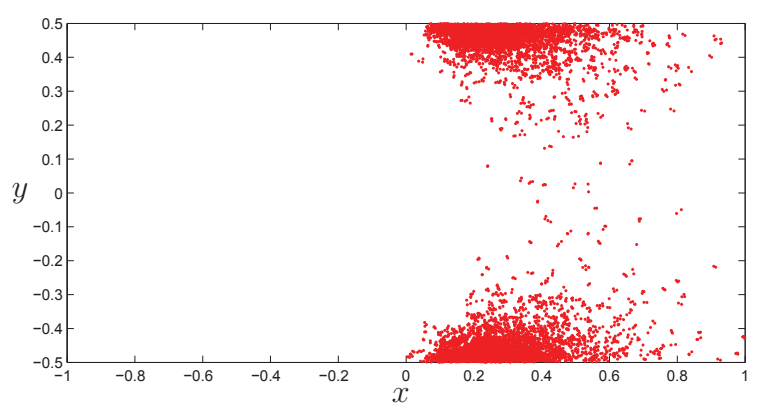

(b) $t=0.585$.

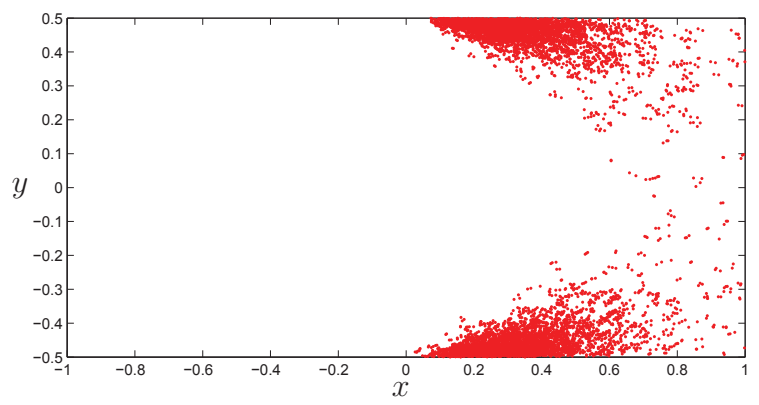

(c) $t=0.85$.

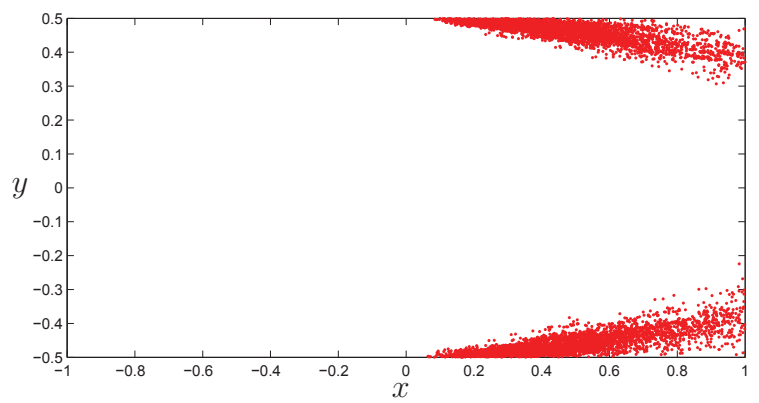

(d) $t=1.66$.

Figure 4-4: Snapshots of droplet locations at different simulation times. The total number of nucleated droplets is kept up to $N_{\text {total }}=10^{4}$. 


\begin{tabular}{|l|l|}
\hline Molecular weight of DBT $(M=g$ mole & -1 \\
\hline Density of DBT $\left(\mathrm{g} \mathrm{cm} \mathrm{cm}^{-3}\right)$ & 278.35 \\
\hline Surface tension of DBT $\left(\mathrm{J} \mathrm{m} \mathrm{m}^{-2}\right)$ & $\rho_{l}^{*}=1.063-0.000826\left(T^{*}-273.16\right)$ \\
\hline Diffusivity of DBT vapor $\left(\mathrm{cm}^{2} \mathrm{~s}^{-1}\right)$ & $\sigma^{*}=0.001\left(35.3-0.0863\left(T^{*}-273.15\right)\right)$ \\
\hline Saturation mole fraction & $D^{*}=0.0398\left(T^{*} / 273.16\right)^{3 / 2}$ \\
\hline Mean free path of DBT vapor $(\mathrm{cm})$ & $Y_{0}=\exp \left(21.497-11497 / T^{*}\right)$ \\
\hline
\end{tabular}

Table 4.1: Properties of dibutylphthalate (DBT) vapor [40].

saturation. The initial size of newly formed droplets depends on the actual location where the droplet is generated. Local conditions, such as the saturation ratio $S_{r}$, temperature $T^{*}$ and surface tension $\sigma^{*}$ (Cf. equation (4.47)), influence the initial size of the generated droplets. The local values are obtained from linear interpolation and lead to $d_{n u c}^{*}$ on the order of $O\left(10^{-3} \mu \mathrm{m}\right)$. The nucleated droplets initially have a very small size and their motion is mainly driven by the Brownian forcing. As a result a fraction of the generated droplets gets deposited at the walls quite shortly after nucleation. Droplets that do not get deposited at the walls, gradually increase in size and become less sensitive to the Brownian forcing. The mean size of the generated droplets increases as the droplets travel through the channel and vapor condenses on them. The small droplet size of newly generated droplets implies very small time steps such that $\Delta t \ll \tau_{v}$. We adopted $\Delta t=10^{-8}$ in case the Stokes number $\tau_{v} \sim O\left(10^{-7}\right)$ corresponding to $d_{n u c}^{*} \sim O\left(10^{-3} \mu m\right)$ and computed from (recalling the definition of $\left.\tau_{v}\right)$ :

$$
\tau_{v}=\tau_{v}^{*} / t_{r e f}^{*}=\frac{C_{c} \rho_{l}^{*}\left(d_{i}^{*}\right)^{2}}{18 \mu_{c}^{*} t_{r e f}^{*}}
$$

where $\mu_{c}^{*}=2.2 \times 10^{-5} \mathrm{~kg} /(\mathrm{m} \cdot \mathrm{s})$ and $\rho_{l}^{*}=1.063-0.000826\left(T^{*}-273.16\right) \mathrm{g} \mathrm{cm}^{-3}$. This results in $\Delta t / \tau_{v} \sim O\left(10^{-1}\right)$. With increasing droplet size the time step was increased at some restarts during the simulation as well retaining the condition $\Delta t<\tau_{v}$ to ensure a stable solution of the numerical scheme given in (4.60). 

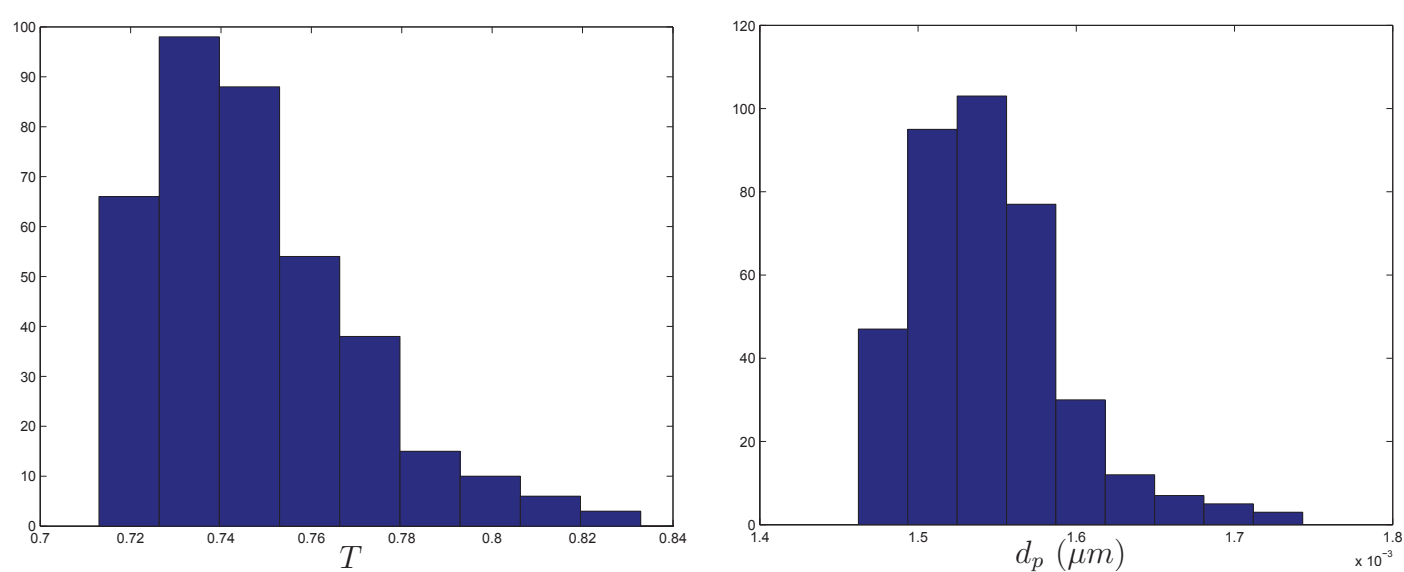

(a) $t=0.58005$.
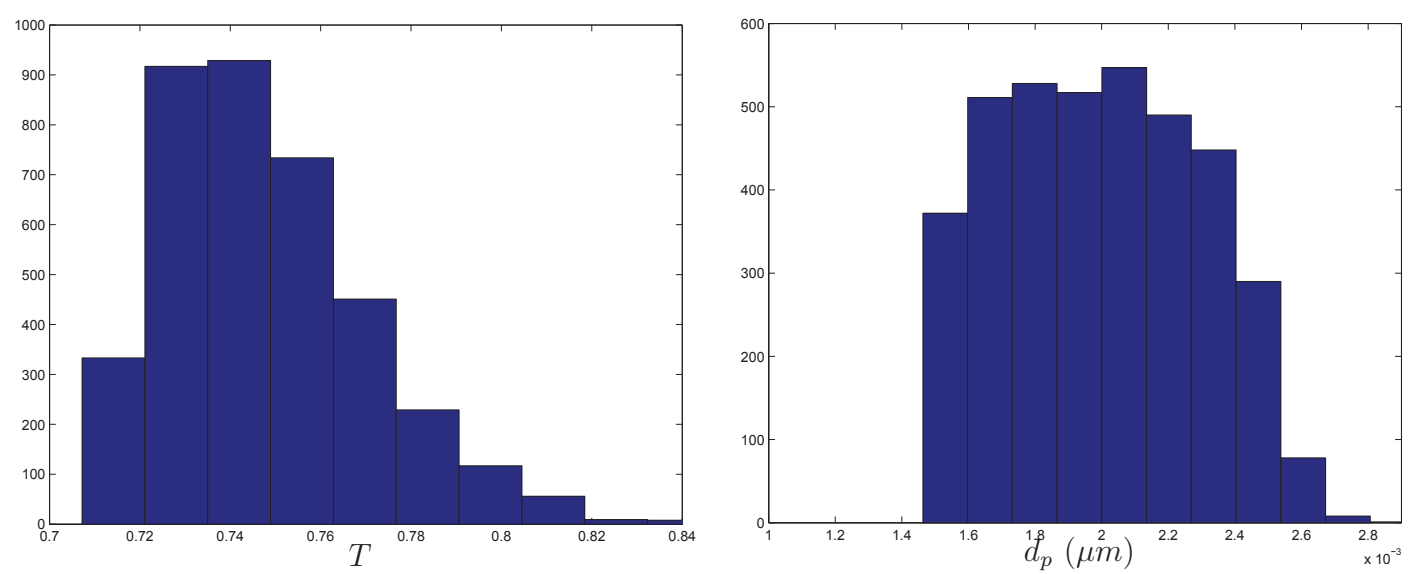

(b) $t=0.585$.
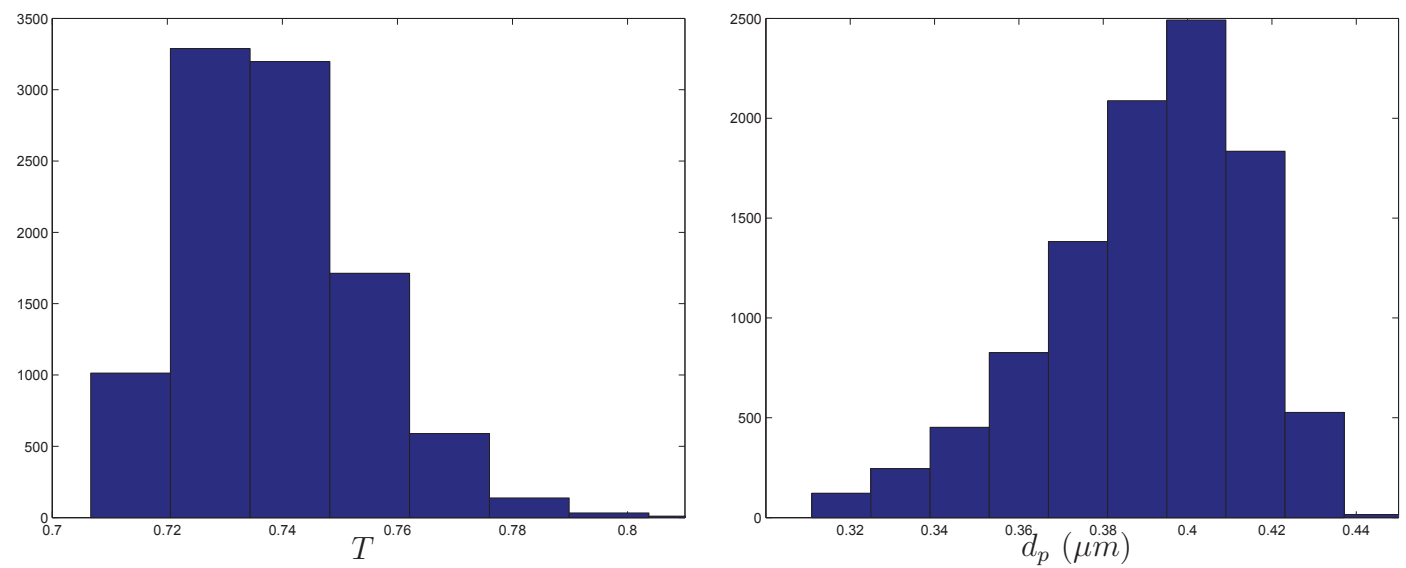

(c) $t=0.85$.

Figure 4-5: Histograms of droplet temperature (left figures) and droplet sizes (right figures) at different simulation times. The total number of nucleated droplets is $N_{\text {total }}=10^{4}$. 


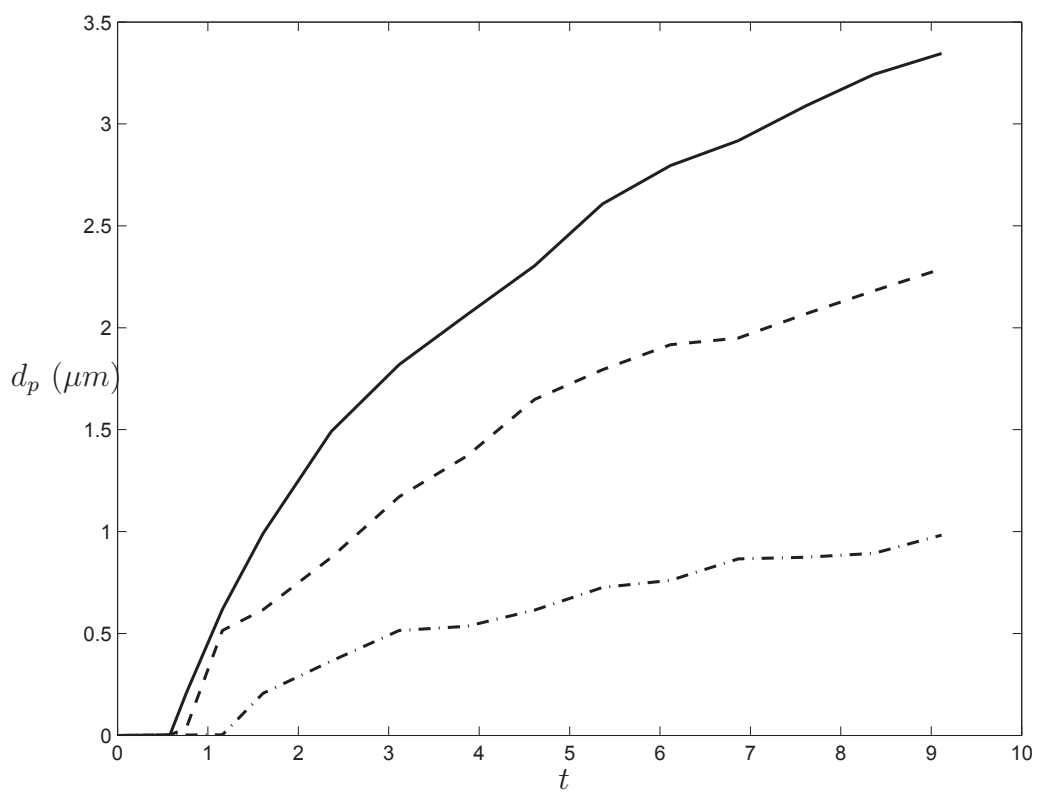

Figure 4-6: Evolution of droplet size as a function of time for droplets that exit the computational domain. This is expressed in terms of percentile $p$ of particle size distribution for $p=10 \%$ (dash-dot), $p=50 \%$ (dash) and $p=90 \%$ (solid).

The particle temperature and size distributions are characterized in Figure 45. Directly after the first droplets get nucleated $(t=0.58005)$ the average droplet temperature is quite close to $T_{2}$, indicating that the droplets predominantly nucleate close to the wall at the stream wise location where cooling was applied. At this moment the nucleated droplet population has sizes close to $d_{n u c}^{*}$. Later in time the temperature shifts to slightly warmer droplets and their sizes are seen to grow quite rapidly. In the well-developed regime, e.g., at $t=0.85$, the temperature of the droplets has settled into a skewed temperature distribution, with a large number of droplets apparently having temperatures quite close to that of the cooling wall. This is consistent with the physical location of the plume of droplets as was shown in Figure 4-4. The sizes have developed to the sub-micron range, i.e., in the time between nucleation and the moment when the first droplets leave the domain the sizes have grown by a factor of about 200 .

A major interest is with the properties of the droplets as these leave the domain. In Figure 4-6 we show the evolution of the mean particle sizes. We observe that the first particles leave the domain at the outlet around $t \approx 0.75$. Subsequently, the 
droplets that arrive at the outflow are larger on average with a time-dependence that looks somewhat similar to a square-root dependence. Also the variation in the sizes at the outflow is seen to grow with time, as is quantified by the $10 \%$ and $90 \%$ percentile lines based on simulation results carried out till $t=9$. For statistically reliable results a larger number of droplets in the system needs to be considered which is a subject for future work.

\subsection{Conclusions}

In this chapter, we described a new computational approach for simulating aerosol droplet formation and growth in a supersaturated system of a gas-vapor mixture. While most treatments of aerosol nucleation and growth via condensation and evaporation follow the Euler-Euler approach, we developed a framework based on the Euler-Lagrange formulation. The nucleation rate which is key in the CNT is adopted in the Euler-Lagrange framework to generate the probability per unit of time and volume to generate a critical cluster. This probability is directly linked to the supersaturation level of the system through the local vapor mass concentration and the temperature. This model was presented in a setting of a laminar flow of air and DBT vapor mixture in a rectangular channel. A supersaturated state of the system was obtained by rapid cooling of the mixture, and property evolution of the generated droplets was monitored. While tracking the droplets exiting the computational domain, under considered conditions a systematic increase of the average droplet diameter from $\approx 0.2 \mu \mathrm{m}$ to $\approx 2.3 \mu \mathrm{m}$ was observed. Provided improved computational capabilities in terms of CPU time, this numerical model can be directly extended to complex geometries, different flow conditions, different temperature and vapor mass concentration fields to study in details droplet formation and growth for a wide range of application areas. 


\section{Chapter 5}

\section{Conclusions and Recommendations}

In this thesis, a computational model was presented for the simulation of aerosol formation and filtration. The model was formulated in the Euler-Lagrange framework in which the trajectories, as well as the heat and mass transfer of individual droplets with their surroundings, were computed time-accurately in velocity, temperature and species concentration fields that were obtained by applying the immersed boundary method to flow in complex domains. By computing the aerosol dynamics as it evolves in a fully resolved background flow field, a detailed understanding of the relevance of the various processes of nucleation, evaporation, condensation and filtration due to Stokes drag and Brownian forcing can be achieved. This allows to extract macroscopic predictions of effective heat and mass transfer as well as effective filtration efficiencies with which future engineering models can be improved. In this final chapter, we draw conclusions and give recommendations for further research.

\section{Filtration}

The problem of filtration of small droplets as these move through the fluid-filled part of a complex porous domain can be formulated conveniently in the EulerLagrange framework. In this case the trajectories of individual droplets can be computed accurately, given the flow field of the carrier gas. We restricted ourselves to rather dilute situations in which the so-called 'one-way' coupling approximation may be adopted [10]. Such situations hold to good approximation for typical situations 
involving the transport of an aerosol such as in the atmospheric boundary layer. For steady flows the one-way coupling allows to separate the problem of determining the flow field from the problem of tracking the motion of inertial droplets in that flow field, which is exactly the approach followed in Chapters 2 and 3.

Filtration in practice often involves the passage of droplets and particles through a complex domain consisting of the fluid-filled part 'cut out' from a solid material. Such domains can take very complex shapes, e.g, in case the solid consists of fibrous material. Due to the inertia of the droplets their trajectories deviate from the streamlines of the carrier gas. As a result, the droplets may collide with the solid-fluid interface, which is the basic step in any mechanical filtration. We followed this approach and treated the filtration of a single droplet in terms of the occurrence of such a collision. If the trajectory of a droplet intersects with the fluid-solid interface, the droplet is considered 'captured' and taken out of the flow. Such simulations can yield the filtration efficiency of a given domain at given flow conditions as function of the droplet size and other parameters. Moreover, the locations at which most of the droplets get deposited can be extracted from such a simulation, potentially to be used in further improvement of designs for mechanical filters.

A key complication in the numerical prediction of filtration of droplets is the potential contamination of the numerically generated trajectories by discretisation errors. In particular for small droplets, i.e., very low Stokes numbers, the difference between actual collision with the surface and collision due to numerical errors is rather small. This could even lead to unphysical filtration of massless particles. For this reason, we developed in Chapter 2 a new near-wall treatment of the motion of droplets which is made consistent with the requirement that massless droplets are certain not to collide with any fluid-solid interface. This treatment was made consistent with the no-slip condition at the interfaces. It was applied to the case that the flow field is obtained from a simple low-order immersed boundary method based on the so-called 'staircase' approximation of the fluid-solid interface. With the help of this approach, the filtration efficiency of a porous medium consisting of a staggered arrangement of square beams was investigated. It was shown that neglecting the no- 
slip consistency in the near-wall particle treatment can result in an overprediction of the filtration efficiency by more than $15 \%$ at practical spatial resolutions. Moreover, the new near-wall treatment was applied to determine the role of droplet inertia in the filtration and a striking non-uniformity of the filtration efficiency as function of the droplet size was found. This was interpreted in terms of specific flow patterns developing within the porous medium and provides a convenient design criterion with which the specificity of the filter for droplets of certain sizes can be improved.

\section{Application to realistic porous media}

In order to predict the overall filtration characteristics of realistic porous media for a wide range of droplet sizes it is important to also incorporate Brownian forcing of the motion of the droplets. This forcing is particularly important for small droplets at high temperatures. In fact, the strength of the Brownian forces is proportional to $\sqrt{k T /\left(m U^{2}\right)}$ where $T$ and $U$ denote typical temperature and velocity scales and $k$ is Boltzmann's constant while $m$ denotes the droplet's mass. In Chapter 3 such a combined model including Stokes drag forcing and Brownian forcing was presented and analysed. The simulation approach was first adopted to flow in a straight cylindrical tube and important dependencies on temperature and droplet sizes were assessed. In particular, Brownian forcing becomes stronger with increasing temperature and decreasing particle size. The filtration efficiency was found to depend uniformly on the particle size, increasing with decreasing particle size. This case of Poiseuille flow served for validation of the computational approach.

The capability of predicting the complete flow, down to the pore scale, that arises in realistic fibrous porous media was exploited to study the filtration characteristics of such a filter. At different temperatures and particle sizes the capturing of droplets onto the solid-fluid interface was simulated and the filtration efficiency recovered. A classical 'V-shaped' filtration curve was observed as function of droplet size with high likelihood of filtration both for sufficiently small (dominant diffusion) as well as for sufficiently large (dominant inertial effects) droplets. The droplet sizes for which filtration is least effective were found to be in the sub-micron range, consistent with 
the typical ranges found in other filters. This builds confidence in the accuracy of the model and motivates its use in the study and optimisation of mechanical filters. Particularly, the recovery of information such as where in the domain the dominant filtration takes place is essential in case filtration characteristics need to be improved for certain size classes. In the example of the fibrous filter we found considerable non-uniformity in the deposition of droplets onto the solid with higher concentrations mainly in the larger pores in the domain.

\section{Aerosol Formation}

Filtration of already formed aerosol droplets is an indirect way of controlling some aspects of the aerosol that eventually emanates from the process. A more direct way involves control over the conditions at which the aerosol actually forms, in an attempt to determine composition and size of the droplets, which is relevant in various consumer products from the food and cosmetics industry and in several medical applications dealing with the respiratory system. This involves a direct coupling of the fluid flow in a certain domain with processes of nucleation, evaporation and condensation. We restricted ourselves to single-species aerosols. Typically, nucleation is treated on the basis of so-called classical nucleation theory (CNT) which requires locally a supersaturated vapour state as condition for the nucleation of so-called 'critical clusters', as the first step in the formation of larger droplets. Such critical clusters arise from statistical fluctuations in a sufficiently dense vapour and are treated as having a certain size at the very onset. A 'virgin' aerosol droplet emerges in CNT at scales of a few nano-meters, which implies that its dynamics is largely dominated by Brownian forces. Subsequent growth of the size of such a droplet can arise from further condensation of vapour molecules onto the droplet, thereby also depleting the local vapour concentration and ultimately implying a mechanism that will stop further nucleation and condensation if the local conditions equilibrate.

While most treatments of aerosol nucleation and growth via condensation and evaporation follow the Euler-Euler framework, we developed a more fundamental approach based on the Euler-Lagrange formulation. The nucleation rate which is key 
in CNT is adopted in the Euler-Lagrange framework as the probability per unit of time and volume to generate a critical cluster. This link can be readily implemented in a computational model in which the local vapour concentration and temperature are used to determine the degree of supersaturation and correspondingly to evaluate the nucleation rate $J$. The number of critical clusters that would be actually generated per grid cell of volume $\Delta V$ and per time step $\Delta t$ is then given by $\xi=J \Delta V \Delta t$. Well-resolved simulations imply $\xi \ll 1$, which is hence interpreted as the probability of actually generating such a cluster at a randomly chosen location in the grid cell.

\section{Application to aerosol nucleation, evaporation and condensation}

The new Euler-Lagrange model for aerosol formation in a supersaturated vapour was adopted to flow in a straight cylinder. For that purpose laminar flow was simulated in a domain into which a vapour flows at sub-critical conditions becoming supersaturated somewhere downstream as a result of rapid cooling of the cylinder wall. This canonical setting was simulated in detail, including both Stokes drag forcing and Brownian forcing of the droplets that were actually formed in the domain. In addition, the droplets were allowed to exchange heat and mass with the surrounding, as a result of which the balance between Stokes and Brownian influences changes with 'maturity' of the aerosol droplets. The interplay of these mechanisms results in a detailed balance expressed in terms of the size distribution of the droplets emanating from the end of the cylindrical domain. Through simulation we were able to compute the properties of the aerosol at the end of the tube for a prototype situation in which the temperature drop associated with the cooling was $1: 0.7$. This establishes the relevance of the Euler-Lagrange framework for aerosol simulations in case fully resolved conditions are available.

\section{Recommendations}

At the end of this thesis, we briefly review main directions into which further research is required to develop the complete simulation strategy to the level that it could become relevant to industrial fluid engineering involving aerosol formation and 
filtration. We list some key elements and give motivations for each.

- Improvement of immersed boundary method to second order: the current immersed boundary method adopts the 'staircase' approximation for the fluid-solid interface. While this is acceptable for the prediction of the fluid flow, more subtle aspects such as heat and mass transfer across the interface can not be predicted reliably. It is required to improve this aspect so that more accurate results can be obtained at lower resolutions and, more importantly, that a wider range of processes, among which conjugate heat and mass transfer, can be dealt with. A possible route towards this could be the inclusion of sub-grid forcing or an approximate cut-cell method.

- Parallel processing of Euler-Lagrange model in real geometries: a clear restriction in the current computational model is the fact that parallel processing is not yet realised. This is a technical improvement that would make it possible to use larger computational resources to simulate more detailed pore-scale models and flows.

- Application of simulation for filtration optimisation. The method developed in Chapters 2 and 3 holds promise toward simulation support for improved designs of mechanical filters. Specific configurations of fibers were shown to display characteristic filtration properties. It appears natural to then adopt this approach and address the 'inverse' problem of finding a fiber configuration with optimal properties with respect to filtration of certain size classes.

- An extensive parameter study should be performed to identify the main parameters playing a role in the aerosol generation, evolution and filtration. For example, quantify the dependency of the filtration rate on flow speed, on vapour mass fraction at the inflow, on the size and inner shape of the filter and on the temperature cooling rate. 


\section{Bibliography}

[1] J. Akedo. Room temperature impact consolidation (rtic) of fine ceramic powder by aerosol deposition method and applications to microdevices. Journal of Thermal Spray Technology, 17(2):181-198, 2008.

[2] P. Angot, C.-H. Bruneau, and P. Fabrie. A penalization method to take into account obstacles in incompressible viscous flows. Numer. Math., 81:497-520, 1999.

[3] R. Becker and W. Dring. Kinetische behandlung der keimbildung in äbersättigten dämpfen. Ann. Phys., 416:719752, 1935.

[4] A. Bürkholz. Aerosol separation on cylinders exposed to flow-comparison of measured and theoretical data. J. Aerosol Sci., 9:199-207, 1977.

[5] S. Chandrasekhar. Stochastic problems in physics and astronomy. Reviews of Modern Physics, 15(1):1-89, 1943.

[6] S. Chen, C. S. Cheung, C. K. Chan, and C. Zhu. Numerical simulation of aerosol collection in filters with staggered parallel rectangular fibres. Comput. Mech., $28: 152-161,2002$.

[7] C. Crow, M. Sommerfeld, and Y. Tsuji. Multiphase flow with droplets and particles. CRC Press, 1998.

[8] E. Cunningham. On the velocity of steady fall of spherical particles through fluid medium. Proceedings of the Royal Society of London. Series A, 83(563):357-365, 1910. 
[9] J. de la Mara Fernández, B. L. Halpern, and J. A. Wilson. Inertial impaction of heavy molecules. J. Fluid. Mech., 149:217-233, 1984.

[10] S. Elghobashi and G. C. Truesdell. On the two-way interaction between homogeneous turbulence and dispersed solid particles. i: Turbulence modification. Phys. Fluids A, 5:1790-1801, 1993.

[11] E.A. Fadlun, R. Verzicco, P. Orlandi, and J. Mohd-Yusof. Combined immersedboundary finite-difference methods for three-dimensional complex flow simulations. Journal of Computational Physics, 161(1):35 - 60, 2000.

[12] B. Fardi and B. Y. H. Liu. Efficiency of fibrous filters with rectangular fibers. Aerosol Sci. Tech., 17(1):45-58, 1992.

[13] J. H. Ferzinger and M. Perić. Computational methods for fluid dynamics. Springer, third edition, 2002.

[14] S. K. Friedlander. Theory of aerosol filtration. Ind. Eng. Chem., 50(8):1161$1164,1958$.

[15] Tong Gao, Yu-Heng Tseng, and Xi-Yun Lu. An improved hybrid cartesian/immersed boundary method for fluidsolid flows. International Journal for Numerical Methods in Fluids, 55(12):1189-1211, 2007.

[16] Yang Ge and L.-S. Fan. 3-d modeling of the dynamics and heat transfer characteristics of subcooled droplet impact on a surface with film boiling. International Journal of Heat and Mass Transfer, 49(2122):4231 - 4249, 2006.

[17] F. Gelbard and J. H. Seinfeld. The general dynamic equation for aerosols. theory and application to aerosol formation and growth. Journal of Colloid and Interface Science, 68(2):363 - 382, 1979.

[18] H. F. George and G. W. Poehlein. Capture of aerosol particles by spherical collectors electrostatic, inertial, inetrception and viscous effects. Envir. Sci. Technol., 8:46-49, 1974. 
[19] B. J. Geurts, P. Pratte, S. Stolz, R. Stabbert, V. Poux, M. Nordlund, and Ch. Winkelmann. Penetration of sub-micron aerosol droplets in composite cylindrical filtration elements. Int. J. Heat Fluid Flow, 32(1):261 - 272, 2011.

[20] L. Ghazaryan, D.J. Lopez Penha, S. Stolz, A.K. Kuczaj, and B.J. Geurts. Noslip consistent immersed boundary particle tracking to simulate impaction filtration in porous media. International Journal for Numerical Methods in Fluids, 73(7):615-636, 2013.

[21] Anvar Gilmanov and Fotis Sotiropoulos. A hybrid cartesian/immersed boundary method for simulating flows with 3d, geometrically complex, moving bodies. Journal of Computational Physics, 207(2):457 - 492, 2005.

[22] D. Gupta and M. H. Peters. A brownian dynamics simulation of aerosol deposition onto spherical collectors. Journal of Colloid and Interface Science, 104(2):375 - 389, 1985.

[23] W. C. Hinds. Aerosol Technology: properties, behavior, and measurement of airbone particles. John Wiley and Sons Inc, second edition, 1999.

[24] Nan-Hung Hsieh and Chung-Min Liao. Assessing exposure risk for dust storm events-associated lung function decrement in asthmatics and implications for control. Atmospheric Environment, 68(0):256 - 264, 2013.

[25] W. F. Weiss IV, T. K. Hodgdon, E. W. Kaler, Ab. M. Lenhoff, and C. J. Roberts. Nonnative protein polymers: Structure, morphology, and relation to nucleation and growth. Biophysical Journal, 93(12):4392 - 4403, 2007.

[26] E. Javaheri and W. H. Finlay. Numerical simulation of flocculation and transport of suspended particles: Application to metered-dose inhalers. International Journal of Multiphase Flow, 64(0):28 - 34, 2014.

[27] L. Koblinger and W. Hofmann. Monte carlo modeling of aerosol deposition in human lungs. part i: Simulation of particle transport in a stochastic lung structure. Journal of Aerosol Science, 21(5):661 - 674, 1990. 
[28] E. Kreyzig. Introductory functional analysis with applications. John Wiley and Sons Inc, 1978.

[29] F. Kuwahara, M. Shirota, and A. Nakayama. A numerical study of interfacial convective heat transfer coefficient in two-energy equation model for convection in porous media. Int. J. Heat Mass Transfer, 44:1153-1159, 2001.

[30] D. J. Lopez Penha. Simulating Microtransport in realistic porous media. $\mathrm{PhD}$ thesis, University of Twente, Netherlands, September 2012.

[31] D. J. Lopez Penha, B. J. Geurts, S. Stolz, and M. Nordlund. Computing the apparent permeability of an array of staggered square rods using volumepenalization. Comput. Fluids, 51:157-173, 2011.

[32] J. Lyyrnen, J. Jokiniemi, E. I. Kauppinen, and J. Joutsensaari. Aerosol characterisation in medium-speed diesel engines operating with heavy fuel oils. Journal of Aerosol Science, 30(6):771 - 784, 1999.

[33] T.B. Martonen and I. Katz. Deposition patterns of polydisperse aerosols within human lungs. International Journal of Multiphase Flow, 6(4):251-274, 2009.

[34] M. R. Maxey and J. J. Riley. Equation of motion for a small rigid sphere in a nonuniform flow. Phys. Fluids, 26(4), 1988.

[35] J. Mikhal. Modeling and simulation of flow in cerebral aneurysms. PhD thesis, University of Twente, Netherlands, October 2012.

[36] R. Mittal and G. Iaccarino. Immersed boundary methods. Annu. Rev. Fluid Mech., 37:239-261, 2005.

[37] Parviz Moin and John Kim. Numerical investigation of turbulent channel flow. Journal of Fluid Mechanics, 118:341-377, 1982.

[38] A. Nakayama, F. Kuwahara, and T. Hayashi. Numerical modelling for threedimensional heat and fluid flow through a bank of cylinders in yaw. J. Fluid Mech., 498:139-159, 2004. 
[39] Elijah P. Newren, Aaron L. Fogelson, Robert D. Guy, and Robert M. Kirby. Unconditionally stable discretizations of the immersed boundary equations. Journal of Computational Physics, 222(2):702 - 719, 2007.

[40] H. V. Nguyen, K. Okuyama, T. Mimura, Y. Kousaka, R. C. Flagan, and J. H. Seinfeld. Homogeneous and heterogeneous nucleation in a laminar flow aerosol generator. Journal of Colloid and Interface Science, 119(2):491 - 504, 1987.

[41] M. Nordlund, D. J. Lopez Penha, S. Stolz, A.K. Kuczaj, Ch. Winkelmann, and B. J. Geurts. Permeability of anisotropic structured porous media. submitted, 2011.

[42] A. J. Pesthy, R. C. Flagan, and J. H. Seinfeld. Theory of aerosol formation and growth in laminar flow. Journal of Colloid and Interface Science, 91(2):525 $545,1983$.

[43] A. Prem and M. J. Pilat. Calculated particle collection efficiencies by single droplets considering inertial impaction, Brownian diffusion and electrostatics. Atm. Env., 12:1981-1990, 1978.

[44] A. Prosperetti and G. Tryggvason. Computational methods for multiphase flow. Cambridge University Press, 2007.

[45] H. R. Pruppacher and J. D. Klett. Microphysics of clouds and precipitation. Atmospheric and oceanographic sciences library. Kluwer Academic Publishers, 1997.

[46] Bandaru V. Ramarao, Chi Tien, and S. Mohan. Calculation of single fiber efficiencies for interception and impaction with superposed brownian motion. Journal of Aerosol Science, 25(2):295 - 313, 1994.

[47] P. C. Raynor. Single-fiber interception efficiency for elliptical fibers. Aerosol Sci.Tech., 42(5):357-368, 2008. 
[48] J. A. Rice. Mathematical Statistics and Data Analysis. Duxbury Press, 3rd edition, 2001.

[49] A. Sarthou, S. Vincent, J. P. Caltagirone, and Ph. Angot. Eulerianlagrangian grid coupling and penalty methods for the simulation of multiphase flows interacting with complex objects. International Journal for Numerical Methods in Fluids, 56(8):1093-1099, 2008.

[50] H. Shen, S. Nutt, and D. Hull. Direct observation and measurement of fiber architecture in short fiber-polymer composite foam through micro-CT imaging. Compos. Sci. Technol., 64(1314):2113 - 2120, 2004.

[51] C. Shin. Numerical simulation for particle penetration depth distribution in deep bed filtration. Chem. Eng. Technol., 29(8):905-909, 2006.

[52] C. Terzano, F. Di Stefano, V. Conti, E. Graziani, and A. Petroianni. Air pollution ultrafine particles: toxicity beyond the lung. Eur Rev Med Pharmacol Sci., 14(10):809821, 2010.

[53] Yu-Heng Tseng and Joel H. Ferziger. A ghost-cell immersed boundary method for flow in complex geometry. J. Comput. Phys., 192(2):593-623, 2003.

[54] Gordon J. Van Wylen and Richard E. Sonntag. Fundamentals of classical thermodynamics / Gordon J. Van Wylen and Richard E. Sonntag. Wiley New York, 3rd ed., si version. edition, 1985.

[55] A.E.P. Veldman, J. Gerrits, R. Luppes, J.A. Helder, and J.P.B. Vreeburg. The numerical simulation of liquid sloshing on board spacecraft. Journal of Computational Physics, 224(1):82 - 99, 2007.

[56] R. W. C. P Verstappen and A. E. P. Veldman. Symmetry preserving discretization of turbulent flow. J.Comput. Phys., 187:343-368, 2003.

[57] M. Volmer and A. Weber. Nucleus formation in supersaturated systems. $Z$. physik. Chem., 119:277 - 301, 1926. 
[58] A. W. Vreman, B. J. Geurts, N. G. Deen, J. A. M. Kuipers, and J .G. M. Kuerten. Two- and four-way coupled Euler-Lagrangian large-eddy simulation of turbulent particle-laden channel flow. Flow, Turbulence and Combustion, 82:47-71, 2009.

[59] Z. Zhang and C. Kleinstreuer. Airflow structures and nano-particle deposition in a human upper airway model. Journal of Computational Physics, 198(1):178 $-210,2004$.

[60] Hong Zhao, Jonathan B. Freund, and Robert D. Moser. A fixed-mesh method for incompressible flowstructure systems with finite solid deformations. Journal of Computational Physics, 227(6):3114 - 3140, 2008. 



\section{Curriculum Vitae}

Lilya Ghazaryan was born on 3 August 1984 in Yerevan, Armenia.

- 2012-present Scientist-innovator at TNO, department of Earth and Environmental sciences.

- 2008 - $2012 \mathrm{PhD}$ student, department of Applied Mathematics, University of Twente, Netherlands.

PhD thesis: Aerosol dynamics in porous media.

- 2007 - 2008 M.Sc. in Industrial and Applied Mathematics, Johannes Kepler University, Linz, Austria (Second Part of Master program).

M.Sc. thesis: Hermitian and Skew-Hermitian Splitting Solvers and Preconditioners: Applications to Symmetric and Indefinite Problems.

- 2006 - 2007 M.Sc. in Industrial and Applied Mathematics, Eindhoven University of Technology, Netherlands (First Part of Master program).

- 2001- 2005 B.Sc. in Informatics and Applied Mathematics, Yerevan State University (YSU), Yerevan, Armenia. 